
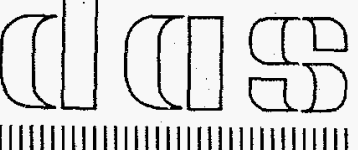

\title{
THE SPRITE AND POS-SPRITE USER REPORT \\ AN EXTENSIBLE CALCULATION OF PARTICLE, POSITRON AND ELECTRON IMPLANTATION IN SOLIDS
}

BNL-64884

RECEIVED

Informal Report

DEC 031997

OSTI

K. A. Ritley

Department of Physics and Materials Research Laboratory

University of Mllinois at Urbana-Champaign

1110 W. Green Street, Urbana, IL 61801-3080

V. J. Ghosh, K. G. Lynn ${ }^{a}$, M. McKeown and D. O. Welch Department of Physics and materials Science Division

Brookhaven National Laboratory, Upton, NY 11973-5000

(a) Present address: Department of Physics

Washington State University, Pullman, WA 99164

October 20, 1997

\author{
Materials Science Division \\ DEPARTMENT OF APPLIED SCIENCE
}

BROOKHAVEN NATIONAL LABORATORY UPTON; LONG ISLAND, NEW YORK 11973 


\title{
THE SPRITE AND POS-SPRITE USER REPORT
}

\section{An Extensible Calculation of Particle, Positron and Electron Implantation In Solids}

\author{
K. A. Ritley \\ Department of Physics and Materials Research Laboratory \\ University of Illinois at Urbana-Champaign \\ 1110 W. Green Street, Urbana, IL 61801-3080
}

V. J. Ghosh, K. G. Lynn ${ }^{a}$, M. McKeown and D. O. Welch

Department of Physics and Materials Science Division

Brookhaven National Laboratory, Upton, New York 11979

(a) Present address: Department of Physics

Washington State Oniversity, Pullman, WA 99164

January 1997

DISTRBUTION OF THS DOCUMENT IS UNTHEME ph

This manuscript has been authored under contract DE-AC02-76CH00016 with the U.S. Department of Energy. Accordingly, the U.S. Government retains a non-exclusive, royaltyfree license to publish or reproduce the published form of this contribution, or allow others to do so, for U.S. Government purposes. 


\section{DISCLAIMER}

This report was prepared as an account of work sponsored by an agency of the United States Government. Neither the United States Government nor any agency thereof, nor any of their employees, makes any warranty, express or implied, or assunes any legal liability or responsibility for the accuracy, completeness, or usefulness of any information, apparatus, product, or process disclosed, or represents that its use would not infringe privately owned rights. Reference herein to any specific commercial product, process, or service by trade name, trademark, manufacturer, or otherwise, does not necessarily constitute or imply its endorsement, recommendation, or favoring by the United States Government or any agency, contractor, or subcontractor thereof. The views and opinions of authors expressed herein do not necessarily state or reflect those of the United States Government or any agency, contractor, or subcontractor thereof. 


\section{DISCLAIMER}

This report was prepared as an account of work sponsored by an agency of the United States Government. Neither the United States Government nor any agency thereof, nor any of their employees, makes any warranty, express or implied, or assumes any legal liability or responsibility for the accuracy, completeness, or usefulness of any information, apparatus, product, or process disclosed, or represents that its use would not infringe privately owned rights. Reference herein to any specific commercial product, process, or service by trade name, trademark, manufacturer, or otherwise does not necessarily constitute or imply its endorsement, recommendation, or favoring by the United States Government or any agency thereof. The views and opinions of authors expressed herein do not necessarily state or reflect those of the United States Government or any agency thereof. 


\section{DLSCANMIXR}

Portions of this docment may be illegibie in electronic image products. Images are produced from the best available origion docomerert 


\section{PREFACE}

This document describes a series of programs for performing calculations relevant to the problem of understanding the behavior of a stream of particles incident on solids.

As with any body of work as extensive as that described in this report, the number of people deserving of credit for their contributions to this work is large. It gives us great pleasure to acknowledge in this report the many individuals who have contributed to this project over the years.

In particular, we thank Jim Davenport and Mike Weinert of Brookhaven National Laboratory for invaluable assistance with theoretical aspects of the calculation. We also thank Yuan Kong, Xiao-Wei Wu, Marc Weber, P.Asoka Kumar and Jane Throwe for their contributions to the code. We also thank E. Gramsch, N. Ozturk, T. McMahon, T. McMullen, B. Nielson, R. Nieminen, J. Oliva, P. Simpson and W. Williamson.

Finally, it is a pleasure to thank Virginia Metze, Elena Pourmal, and Brian Rogers of the Materials Research Laboratory Center for Computation at the University of Illinois, and Harold Berry of the Applied Mathematics Division of Brookhaven National Laboratory, for the invaluable technical assistance they have provided. 


\section{Table of Contents}

DISCLAIMER $\ldots \ldots \ldots \ldots \ldots \ldots \ldots \ldots$ ii

PREFACE $\ldots \ldots \ldots \ldots \ldots \ldots \ldots \ldots \ldots \ldots \ldots \ldots \ldots \ldots$ iii

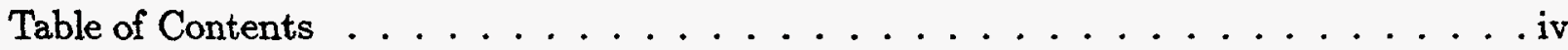

List of Figures $\ldots \ldots \ldots \ldots \ldots \ldots \ldots \ldots \ldots$ vi

List of Tables $\ldots \ldots \ldots \ldots \ldots \ldots \ldots \ldots \ldots \ldots \ldots \ldots$ vilii

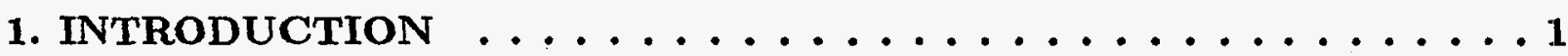

1.1 Intent of This Report $\ldots \ldots \ldots \ldots \ldots$

1.2 Summary of Capabilities . . . . . . . . . . . . . . 1

1.3 Summary of Limitations $\ldots \ldots \ldots \ldots \ldots$

1.4 The Need for SPRITE $\ldots \ldots \ldots \ldots \ldots \ldots$

1.5 The Need for POS-SPRITE $\ldots \ldots \ldots \ldots \ldots$

1.6 History of SPRITE and POS-SPRITE . . . . . . . . . . . 4

1.7 How This Report is Organized . . . . . . . . . . . . . 5

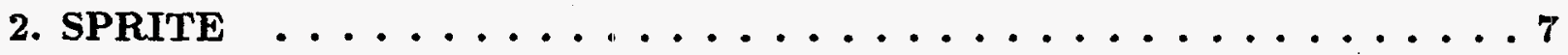

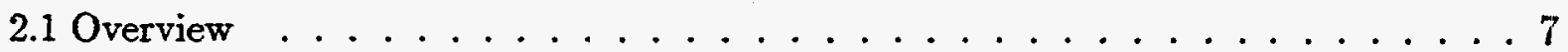

2.2 The Monte-Carlo Procedure . . . . . . . . . . . . . 8

2.3 User-Input Scattering Information $\ldots \ldots \ldots$

2.4 Initial Conditions . . . . . . . . . . . . . . . . . . . . 14

2.4.1 Description of the Solid . . . . . . . . . . . . . . 14

2.4.2 Description of the Particles . . . . . . . . . . . . . . 19

2.5 Physical Dimensions and Units . . . . . . . . . . . . . 20

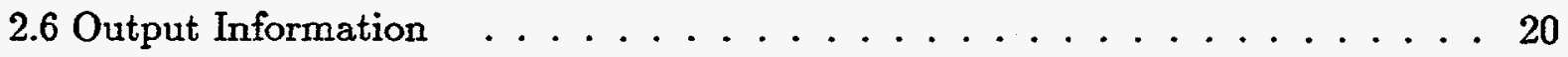

3. Examples of SPRITE Data $\ldots \ldots \ldots \ldots \ldots$

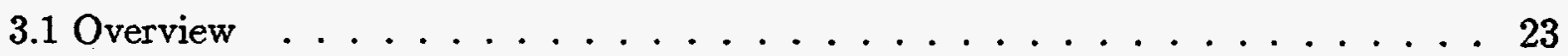

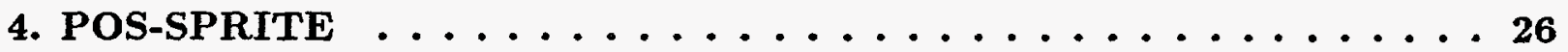

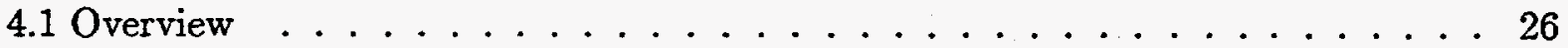

4.2 Elastic Scattering . . . . . . . . . . . . . . . . 29

4.3 Inelastic Scattering . . . . . . . . . . . . . . . 33 


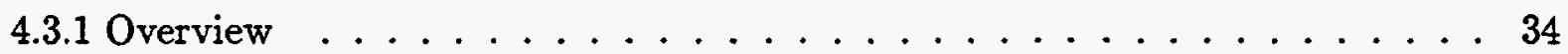

4.3.2 Calculation of Cross Sections . . . . . . . . . . . . 34

4.3.2.1 K-shell core-level scattering . . . . . . . . . . . . . . . . . 34

4.3.2.2 Non-K-shell core-level scattering . . . . . . . . . . . . 35

4.3.2.3 Valence-level inelastic scattering . . . . . . . . . . . . 36

4.3.3 Calculation of Energy Loss . . . . . . . . . . . . . . . . 38

4.3.3.1 Core-level Energy Loss _ . . . . . . . . . . . . . . . . . 38

4.3.3.2 Valence-level Energy Loss . . . . . . . . . . . . . . . . . . . . 39

4.3.4 Calculation of Scattering Angle . . . . . . . . . . . . . 42

4.3.4.1 Core-level Scattering Angle . . . . . . . . . . . . . . . . 42

4.3.4.2 Valence-level Scattering Angle . . . . . . . . . . . . . . 43

4.4 POS-SPRITE Simulation Control File . . . . . . . . . . . . 48

Appendix A. The Structure of SPRITE $\ldots \ldots \ldots \ldots$

A.1 The Program Structure $\ldots \ldots \ldots \ldots$. . . . . . . . 50

A.2 Global Variables . . . . . . . . . . . . . . . . 50

Appendix B. Incorporating a User-Defined Subroutine $\ldots \ldots \ldots$

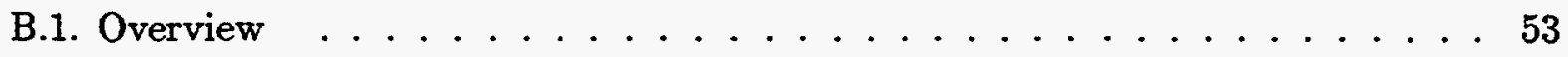

B.2. Example: Single Mechanism . . . . . . . . . . . . . 54

B.2. Example: Multiple Mechanisms . . . . . . . . . . . . . . 55

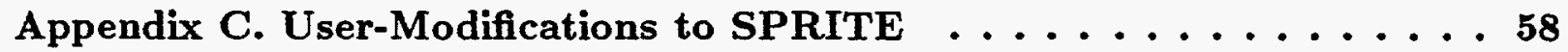

C.1. New criteria for terminating the simulation . . . . . . . . . 58

C.2. Altering the Output Information . . . . . . . . . . 60

C.3. Interfacial Effects . . . . . . . . . . . . . . . 61

Appendix D. Useful Information $\ldots \ldots \ldots \ldots$

Appendix E. Sample Output Information $\ldots \ldots \ldots 68$

References ......................... 76 


\section{List of Figures}

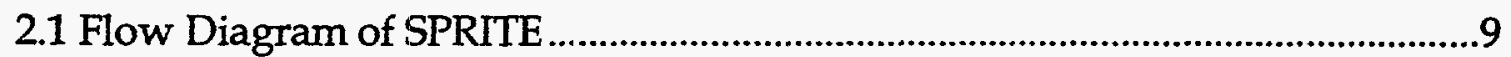

2.2 Definition of particle trajectory .............................................................................11

2.3 Block diagram of the required files ......................................................................11

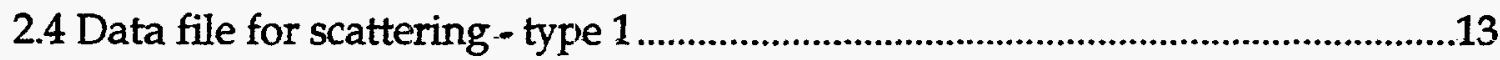

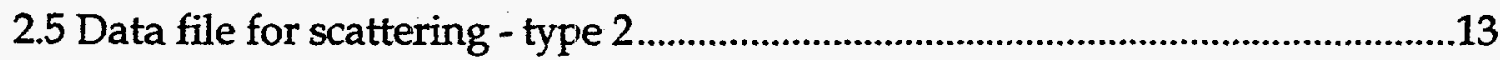

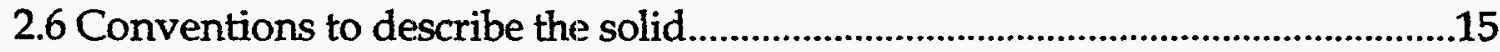

2.7 Simulation control file, single layer..................................................................16

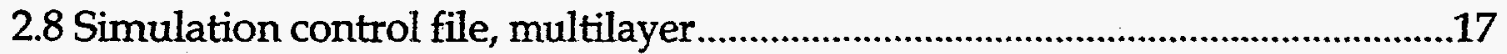

2.9 Particle initial condition file .............................................................................18

2.10 Sample of dębugging information...................................................................19

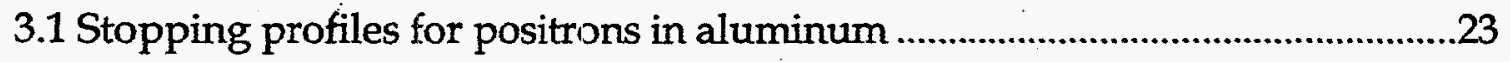

3.2 Fraction of incident positrons backscattered ..........................................................24

3.3 Lateral and depth stopping profiles......................................................................24

3.4 Fraction of reemitted positrons........................................................................25

3.5 Effects of a work function on positron reémission............................................25

4.1 Block diagram of POS-SPRITE files...................................................................27

4.2 Differential elastic scattering cross section............................................................30

4.3 Total elastic scattering cross section .....................................................................31

4.4 Atomic potential for aluminum ...........................................................................33

4.5 Renormalized atomic potential for aluminum .......................................................33

4.6 Cross sections for atomic K-shell scattering ........................................................36

4.7 Gryzinski cross sections for atomic scattering in $\mathrm{Al}$..............................................37

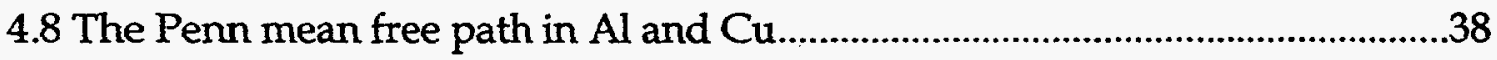

4.9 Energy-dependent inelastic differential cross section ............................................40

4.10 Valence-level inelastic differential cross section ...................................................43

4.11 Vectorial representation of inelastic scattering...................................................43

4.12 Excitation spectrum of the free electron gas .........................................................45 
4.13 Differential cross section for plasmon scattering …...........................................47

4.14 PSPRITE simulation control file......................................................................49

A.1 Main module of the SPRITE program ...............................................................51

C.1 Modified SPRITE subroutine to terminate a trajectory ......................................59

C.2 Variation of a SPRITE subroutine ........................................................................62

D.1 Hierarchical list of SPRITE subroutines...........................................................63

D.2 Block diagram of POS-SPRITE files.................................................................65

E.1 Simulation control file, single layer …..............................................................68

E.2 Particle initial condition file .................................................................................69

E.3 Interaction energy file .................................................................................

E.4 Sample output file ................................................................................................71

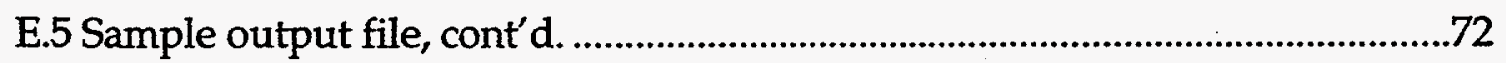

E.6 Sample output file, cont'd. ................................................................................73

E.7 Sample output file, cont'd. ..............................................................................

E.8 Sample output file, cont'd. ...................................................................................75 


\section{List of Tables}

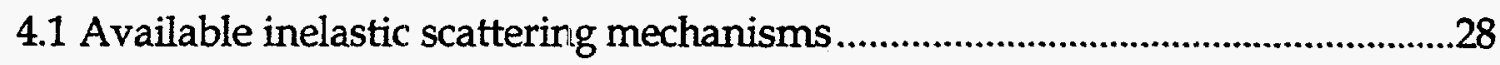

D.1 Functional grouping of SPRITE subroutines ....................................................64

D.2 Global variables in SPRITE, grouped by function ..............................................66

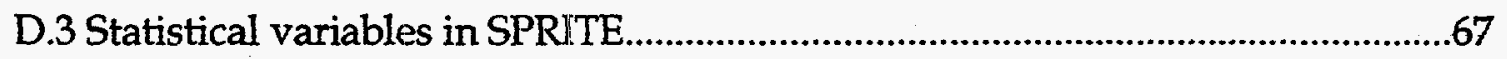




\section{INTRODUCTION}

\subsection{Intent of This Report}

SPRITE (Simulation of Particle Reemission, Implantation and Transmission - Extensible) is a Fortran computer program designed to model the transport of a stream of energetic particles as they scatter through a solid or multilayer. SPRITE is intended to be a user-friendly and easily-extensible "engine" for performing basic transport calculations, and SPRITE incorporates such physics as is required to simulate the transport process, but specifically omits the details of the scattering mechanisms. Information about the scattering mechanisms is supplied by the user, either in the form of external data files or else by a user-constructed subroutine incorporated into SPRITE.

PSPRITE is a Fortran computer program, built around the SPRITE transport "engine" and incorporating physical information necessary for modelling the implantation and thermalization of a stream of positrons or electrons with a solid. The POS-SPRITE suite of programs also includes several additional programs (PATOMIC, PVCALC, PSIGEL, and POSRPA) needed to generate data required by PSPRITE, as well as several programs to facilitate data reduction and analysis. These programs comprise the complete POS-SPRITE package.

This document is intended to be the primary source of information and the only operations manual for SPRITE and the POS-SPRITE family of programs. This information includes the mode of operation of SPRITE, the format of the required and optional file types, as well as information about the output and results of the calculation. Information about installing and running these programs on a variety of computer systems will not be addressed in this report. Such information is rapidly expanding as these programs are adapted to run on different platforms, and thus the user can expect such information to be contained with the source code distribution set.

Detailed information about the calculations and the structure of the POS-SPRITE programs are provided in this report, but detailed "benchmark" comparisons between the output of these calculations and experimental data are an active topic of research, and we refer the reader to the published literature for this information. ${ }^{1-12}$

\subsection{Summary of Capabilities}

The capabilities of SPRITE are briefly summarized as follows.

- Flexibility in specifying initial conditions, including spatial/energetic properties of particles

- Targets include arbitrary infinite or semi-infinite solids, multilayers, heterostructures, etc.

- Ability to easily handle interfacial effects, such as scattering between layers 
- Flexibility in specifying scattering mechanimsms, using static information stored in external data files, or dynamic information (which can be adapted during run-time) obtained from user-constructed subroutines

- Extensive output information, including depth distribution, energy distribution, mean scattering time, statistical information about scattering events, etc.

The PSPRITE calculation is built around the SPRITE transport engine, and as such it has the capabilities listed above. In addition to these, PSPRITE also has the following capabilities:

- User selection and control of the positron-solid or electron-solid scattering mechanisms

- User-control of the scattering neechanisms, including the ability to employ experimental data

- Produce data directly relevant to the subsequent problem of thermal positron diffusion, including stopping profiles and backscattered fractions

\subsection{Summary of Limitations}

The limitations of SPRITE and PSPRITE are briefly summarized as follows.

- Implantation energies used have been less than or equal to $10 \mathrm{keV}$

- The chosen scattering cross sections apply to metals and small band-gap semiconductors, not to wide band-gap seniconductors or insulators

- The routines which update the particle position assume non-relativistic scattering

- No ability to utilize stopping power data; this will be included in a future release of the program

- Only particle-solid interactions are included, not interactions of incident particles with each other

- The model assumes the material is amorphous; no channeling or other effects due to the crystalline structure of the lattice are included

- No particle annihilation-in-flight is considered

\subsection{The Need for SPRITE}

The solution of problems involving the transport of particles through a medium is of profound technological significance. The bulk of the research effort, however, is usually concentrated on the development or refinement of the individual scattering mechanisms which result in the transport, and not on the transport process itself. In fact, a large and diverse collection of transport problems involving physically unrelated scattering mechanisms can be understood and modelled using an equivalent transport theory. Some examples include determining the depth distribution of implanted positrons in solids, and the calculation of the energy distribution of electrons transmitted through thin films.

The theoretical study of transport normally requires a two-fold effort: first, the particlesolid interactions must be calculated; then, these results must be incorporated into a 
transport calculation. SPRITE provides an alternative to this effort. SPRITE offers an easy-to-use and easy-to-modify "engine" for performing transport calculations, in which problem-specific information can be provided by the user, and in which details about the transport calculation can be relegated to SPRITE. Thus, the bulk of the research effort need only be directed toward the details of the particle-solid interaction.

\subsection{The Need for POS-SPRITE}

We briefly review the current status of slow positron experiments, to provide information about the motivation for POS-SPRITE. Positrons are an ideal probe of many phenomena in condensed matter systems. Their size and electric charge is of the same scale as atomic imperfections, making them a sensitive tool for investigating defects. They can be scattered coherently, making them useful for the structural imaging of solids and surfaces. And, upon annihilation with an electron from the host lattice, they can produce a detectable gamma ray signal which depends only on the local environment at the site of annihilation, providing unambigous information from areas which are not easily accessible with other techniques, such as buried interfaces and defects localized in multilayers.

Experimental techniques involving positrons can be roughly classified into two categories: those which are counterparts to specific methods involving electrons, and those which exploit the unique nature of the positron probe. In the former case, positrons usually offer significant advantages over their electron counterparts, although such advantages can easily be outweighed by the difficulty with which adequate sources of positrons can be obtained in the laboratory. For example, low-energy positron diffraction ${ }^{13}$ (LEPD, similar to the well-known LEED) and positron-induced Auger electron spectroscopy ${ }^{14}$ (PAES, similar to AES) can be accomplished with substantially lower beam currents than for the corresponding electron technique. Scattered positrons are easily separated from the secondary electrons (resulting in higher signal-to-noise ratios), surface specificity can be carefully controlled, and problems associated with sample charging can be avoided. We refer further discussion regarding these methods to a recent review..$^{15}$

Our chief concern here lies with providing a theoretical basis for understanding those techniques which exploit the unique nature of the positron. Techniques such as defect profiling begin by implanting a positron into a solid, usually at kinetic energies of a 1$50 \mathrm{keV}$. The positrons lose energy through a variety of inelastic scattering mechanisms, and attain thermal equilibrium with the host lattice. ${ }^{16}$ This occurs in a few picoseconds, quite short compared to the lifetime for positron annihilation. The positrons subsequently diffuse through the lattice in a Bloch-like state, trapping in defects and responding to local fields. ${ }^{17,18}$ This life cycle eventually terminates in one of several ways: the positron can be backscattered or reemitted from the host, it can form Ps and desorb or annihilate, or it can directly annihilate with an electron in the host. ${ }^{19,20}$ Typical diffusion times are on the order of several hundred picoseconds. ${ }^{15}$

The large diffusion time compared with the thermalization time is fortuitous, as it has facilitated data analysis in many experiments by the application of a simple diffusion equation $^{21}$

$$
\frac{\partial N(\mathbf{r}, t)}{\partial t}=D_{+} \nabla^{2} N(\mathbf{r}, t)+\ldots
$$


where $N(\mathbf{r}, t)$ is the number density of positrons, $D_{+}$is the diffusion coefficient, and '...' denotes additional terms, such as depletion through annihilation or Ps formation, drift incurred from local fields, etc. The key to solving such differential equations, however, requires that the initial conditions be known quite accurately, in this case, the spatial distribution of the positrons after energy loss and just prior to diffusion. These initial conditions are usually characterized by the implantation (or stopping) profile, $P(z)$, where $P(z) d z$ is the probability of locating a thermalized positron between $z$ and $z+d z$.

Unfortunately experimental measurements of this quantity are difficult. The first measurements were performed over a decade ago by Mills and Wilson. ${ }^{22}$ These authors studied $\mathrm{Al}$ and $\mathrm{Cu}$; they found that the dependence of the implantation depth on the incident energy could be described by a power law, and they reported the first implantation profiles, obtained by differentiating the measured transmission coefficient of positrons through thin films.

These observations were corroborated shortly thereafter by the first theoretical study of the complete implantation process by Valkealahti and Neiminen. ${ }^{23}$, 24 These authors constructed a simple Monte Carlo model of the process. Elastic effects were accounted for using the method of partial waves. The inelastic effects were considered approximately by using the empirical Gryzinski excitation function, which was originally developed to provide cross section data for the scattering of electrons from atomic cores. Although somewhat deficient in the accuracy of the scattering processes considered, and (more importantly) without significant experimental confirmation, the results of this model have been applied to the description of implantation in a large number of metals, semiconductors, and insulators.

POS-SPRITE is a more sophisticated version of this calculation. It was intended to surpass the shortcomings of the previous calculation and to provide the user with a full complement of scattering mechanisms, as well as the ability to study their individual contribution to the overall results of the calculation. The history of POS-SPRITE is discussed in greater detail in the next section.

\subsection{History of SPRITE and POS-SPRITE}

SPRITE and POS-SPRITE (Version 3.0) are the latest in a series of programs developed by Brookhaven National Laboratory and the University of mlinois.

Beginning in 1986, a project was initiated at Brookhaven National Laboratory to develop a comprehensive Monte-Carlo calculation of positron implantation. The intent was to far surpass the first theoretical work of Valkealahti and Nieminen: to develop a computer model which would include all relevant positron physics, and which would be capable of simulating positron implantation in metals, semiconductors, and insulators. This calculation was intended to be an ab initio model; that is, it contains no arbitrary parameters, aside from the ordinary constants (such as the free-electron radius) which define the material-specific paranaeters. Herewith we provide a brief review of this first calculation. The Monte Carlo segment of the calculation is known as BNLMC; a series of ancilliary programs to provide data for BNLMC were also constructed.

BNLMC is an implementation of the standard Monte Carlo model of particle transport in solids, wherein the positrons are assumed to undergo a succession of discrete scattering events. ${ }^{11}$ The physical observables (such as the stopping profiles, fraction of positrons which 
are backscattered out of the material, time required for thermalization, etc.) are obtained by averaging over a large number of single-particle trajectories through the material.

Unlike the earlier work of Valkealahti and Nieminen, all relevant elastic and inelastic scattering processes are included, using the best available experimental or (when experimental data is lacking) theoretical descriptions of these mechanisms. Elastic scattering is treated using the method of partial waves, in which a positron undergoes scattering from single atomic potentials; these potentials are computed using the relativistic DiracHartree-Fock-Slater method, and are corrected for the approximate effects of the lattice by means of spherical averaging. The inelastic processes include core-level scattering from the $\mathrm{K}$-shell using the results of Kolbenstvedt, which also accounts for Bhabha scattering; and core-level scattering from higher shells using the cross section of Gryzinski and the energy loss results of Rohrlich and Carlson. Valence-level scattering includes collective (plasmon) excitations and single-particle (particle-hole) excitations using the empirical Penn mean free path and energy loss via the standard RPA approach with the Lindhard dielectric response function. The probability of positron annihilation in flight is quite low prior to thermalization, and thus this process is excluded from the model. Effects due to the structure of the lattice (such as defects or channeling) are excluded. BNLMC can chart the progress of positrons in a solid from incident kinetic energies of several $\mathrm{keV}$, to an endpoint energy of $25 \mathrm{eV}$. At energies below this, some of the scattering mechanisms present in the model diverge, and quantum mechanical effects become dominant.

During the development of BNLMC it was realized that, due to the similarity between positron-solid interactions and electron-solid interactions, it was possible (with only a small additional effort) to perform the calculation for both incident positrons and electrons. The necessary formalism was included in BNLMC and thus the BNLMC calculation is valid for both positrons and electrons.

BNLMC was extensively tested and refined over a period of several years, but several problems prevented the widespread distribution of this program. First, the code was not optimized for execution speed, thus its use was restricted to large-scale computer systems. Second, the code was not optimized for user-friendliness. Thus understanding the operation of BNLMC (and the ancilliary programs) and acquainting new users with these programs was difficult. In 1992 it was decided that a modernization of BNLMC would encourage greater use of this program.

In the effort to modernize BNLMC, it became apparent that the calculation involved two separate tasks: a scattering calculation that was independent of the physics associated with the positron-solid interaction, and the calculation of the specific positron-solid interactions. It was further realized that maximum benefit could be gained by formally separating these tasks. This immediately led to the development of the SPRITE calculation, which provides a user-friendly and easy-to-modify front-end for this transport simulation and others; and the PSPRITE calculation, which provides positron-specific information to the SPRITE front-end. The ancilliary programs (which provide data necessary for the Monte Carlo calculation) were also re-written to improve efficiency and to ensure conformance to a single standard (including structure of the code and the details of the user interface).

\subsection{How This Report is Organized}

This report is organized in the following way. In Section 2 the general particle transport 
"engine" SPRITE is described. This includes a full description of the operation of the program, the details of the calculation, and the exact specification of the required files. In Section 3 the positron/electron transport calculation PSPRITE is described, as well as the ancilliary programs which generate data (such as elastic differential scattering cross sections, etc.) required by the calculation. The full details of all positron-solid and electronsolid scattering mechanisms are provided. The Appendices contain detailed information about the structure of SPRITE, as well as information about ways in which the source code can be modified by the user. This information is expected to be of use to users who intend to customize the original program in any way. Note that SPRITE was intended to be user-friendly and easy-to-modify. 


\section{SPRITE}

\subsection{Overview}

Obtaining information about the behavior or particles incident upon or from within a solid is a problem of considerable importance and technological application. It occupies a prominent position in the history of radiation physics. In the seventy years since Bethe's seminal work on the continuous slowing down approximation, the importance of this problem has not subsided. ${ }^{25}$ It has attracted the attention of a diverse sector of the scientific community, and work towards solving this problem has consistantly spurred the development of new experimental techniques and theoretical methods.

An important result of investigations into this problem has been the development of highly-sophisticated computer-based calculations which provide information about irradation and implantation. Numerous calculations have been published to date which are relevant to specific systems, such as electron, neutron and photon irradiation, ion and atomic implantation, etc. We briefly describe several well-known examples: EGS4, a system developed by Nelson and co-workers at Stanford to provide information about high-energy electron implantation in solids (in the energy range from $\mathrm{GeV}$ to $10 \mathrm{keV}$ ), and able to account for arbitrary source and target geometries; ${ }^{26}$ MORSE, a multipurpose neutronphoton transport code, ${ }^{27}$ and TRIM-90, a personal-computer-based calculation which uses experimental stopping powers to provide information about atoms and ions incident on solids in the slab geometry. ${ }^{28,29}$ An excellent review of many of these calculations can be found in Reference 30 .

To date, there have been a large number of calculations concerned with medium- and low-energy ( $50 \mathrm{keV}$ or less) electron implantation in solids. ${ }^{31-40}$ There has recently been a resurgence of interest in these calculations for furthering the development of electron microscopy and electron beam lithography. ${ }^{41,42}$ These calculations provide information about the implantation process, including the depth distribution of electrons which are stopped, the depth distribution of deposited energy, fraction and energy distribution of particles which are backscattered, etc. Many of these calculations employ a similar stochastic transport model to obtain the evolution of the system, and differ only in the system-specific scattering information such as cross section, angular deflection, and energy loss per collision. This similarity between otherwise distinct physical simulations provides the basis for SPRITE.

The SPRITE calculation is a self-contained and "physics-free" computer-based "engine" for performing particle transport calculations. It will accept user-input information about the scattering process (such as the scattering cross sections) and target geometry, and will return information about implantation and backscattering. This calculation differs from previous calculations in one important respect: the computer program which implements this calculation has been optimized wherever possible for user-friendliness and ease of modification, even at the cost of execution speed. Inasmuch as highly-sophisticated 
and complicated computer programs exist to perform detailed calculations for specific systems, it was felt that a real need exists for a simple and easy-to-modify program, expected to be of use in simple cases.

The following subsections will address the functionality and operation of SPRITE, in sufficient detail to permit the user to immediately begin using this program. Although SPRITE has been designed to perform the transport calculation in a manner thought to be most applicable to many circumstances, it is impossible for one program to function in all cases. Thus the SPRITE code has been designed to be easy to modify, even by a casual user with little programming experience. To assist in this regard, the details of the program construction, the names and functions of the global variables, and other relevant information (including examples) about modifying SPRITE are provided in the Appendix.

\subsection{The Monte-Carlo Procedure}

The present work is an adaptation of a standard Monte-Carlo model of particle transport in solids, wherein the particles are assumed to travel at fixed energy through the media and to undergo occasional discrete scattering events, at which times the particle energy is demoted according to the type of scattering event which has been found to occur. The behavior of a beam of particles incident on or from within an arbitrary target is modelled by averaging a large number of single particle trajectories through the material. Using the notation of Reference 31, a trajectory can be denoted by a series of states $\left(E_{n}, \mathbf{r}_{n}, \Omega_{n}\right)$, where $E_{n}, \mathbf{r}_{n}$ and $\Omega_{n}$ represent the energy, position vector and direction cosines of a particle after the $n^{\text {th }}$ scattering event. As a particle travels through the material, it can scatter elastically $\left(E_{n+1}=E_{n}\right)$ or inelastically $\left(E_{n+1}<E_{n}\right)$, and its progress is followed until it meets some criteria for termination of the trajectory. Such criteria may include backscattering from or transmission through the media $\left(r_{z}<0\right.$ or $\left.r_{z}>d\right)$, particle kinetic energy falling below the threshold energy for the calculation ( $E_{n}<E_{\text {stop }}$ ), or some other process (for example, particle annihilation in the case of particles with a finite lifetime).

The scattering states $\left(E_{n}, \mathbf{r}_{n}, \Omega_{n}\right)$ are calculated from a knowledge of the mean energy loss, scattering cross section (or mean free path), and scattering angle of a positron at energy $E$. The particle may by sca.ttered by a number of different mechanisms, as specified by the user, each of which can be characterized by a different scattering cross section $\sigma_{i}$. In general the cross sections will be energy- and material-dependent. The type of scattering event at each collision is determined from the branching ratios computed from these cross sections. The branching ratios $F_{i}(E)$ for the $N$ possible scattering types at energy $E$ form a probability distribution ${ }^{26}$

$$
F_{i}(E)=\frac{\sum_{j=1}^{i} \sigma_{j}}{\sum_{j=1}^{N} \sigma_{j}}
$$

Scattering event types are randomly sampled from this distribution in the following way: given a uniformly-distributed random number $R_{1}$, the scattering type is taken to be the scattering mechanism $i$ which satisfies $F_{i-1}<R_{1}<F_{i+1}$. Once the scattering type has been determined, the energy loss $\Delta E$ and scattering angle $\Omega_{n}$ (taking a random azimuthal angle) are then computed, using information, specific to the scattering mechanism selected 


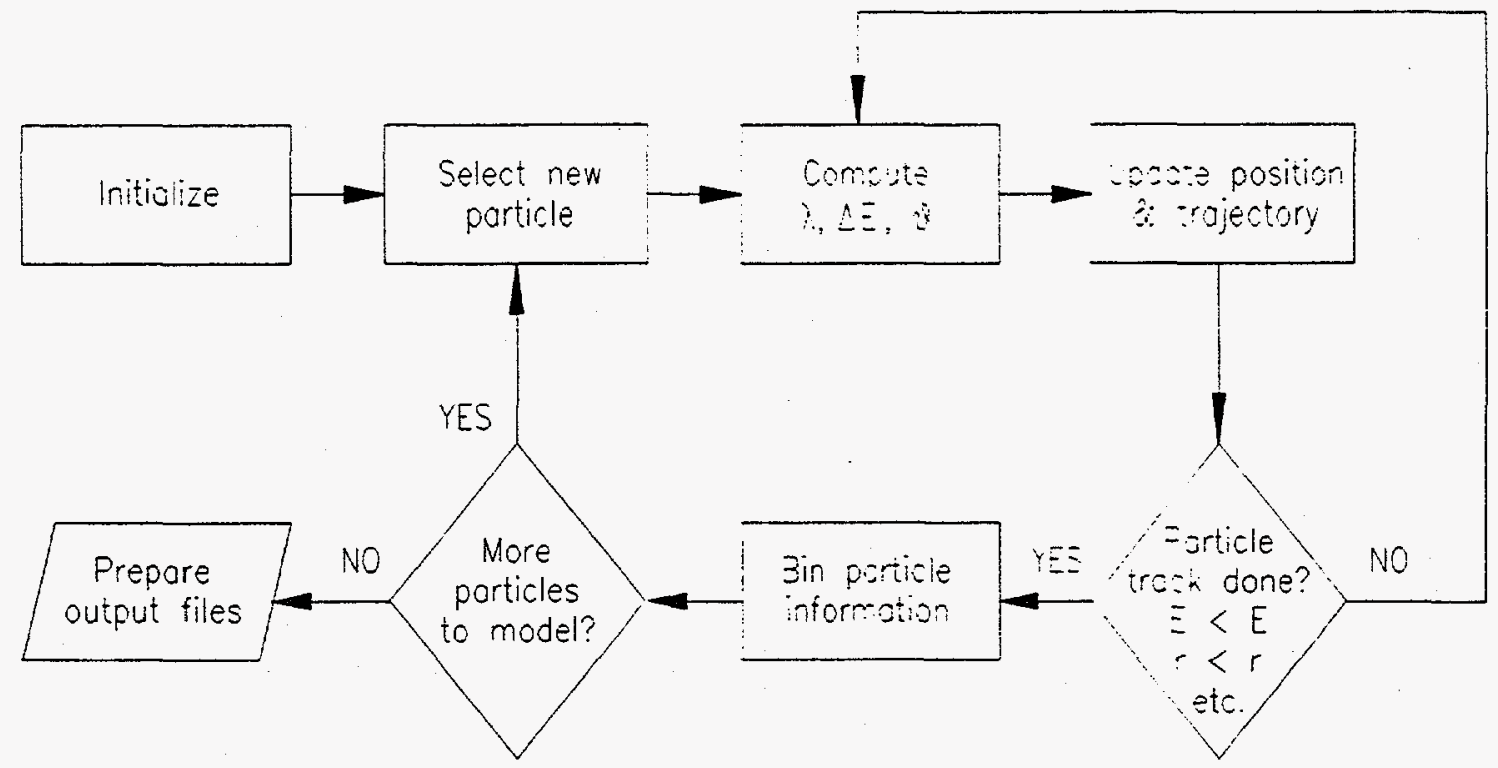

Figure 2.1 Flow-diagram of the scattering process implemented by SPRITE. The track computations for each particle are ended when user-specified criteria (such as particle energy falling below a threshold, or particle ejection from the material) are met.

and supplied by the user and discussed in greater detail below. This process is depicted in Figure 2.1.

We require a method for calculating the distance the particle travels between scattering events. Let $P(t)$ denote the probability that a particle will exist for a time $t$ before scattering, and let wdt denote the probability that a particle will undergo a collision between times $t$ and $t+d t$. A functional form for $P$ can be developed in the following way: The probability that a particle survives to a time $t+d t$ must be equal to the probability that the particle first survives to a time $t$, then continues to survive until the time $t+d t$. The latter lifetime is equal to $1-w d t$ (i.e., the probability that the particle does not scatter between $t$ and $t+d t)$. Thus

$$
P(t+d t)=P(t)(1-w d t)
$$

This leads to the first-order differential equation ${ }^{43}$

$$
\frac{1}{P} \frac{d P}{d t}=-w
$$

which, given that $P(t=0)=1$, has solution

$$
P(t)=e^{-w t}
$$

The mean time between scattering events $\tau$ varies inversely as the collision probability $\omega$, so that the probability that the particle travels a distance $s$ before scattering can be 
written $P(s)=\exp (-s / \lambda)$, where $\lambda$ is the mean free path. Thus the distance the particle has travelled between collisions is computed from

$$
s=-\lambda_{T} \log R_{2}
$$

where $R_{2}$ is a uniformly-distributed random number. The total mean free path $\lambda_{T}$ of the particle when there are $N$ possible scattering events with scattering cross sections $\sigma_{i}, \quad i=1,2, \ldots, N$ is equal to

$$
\lambda_{T}=\frac{A}{N_{A} \rho \sigma_{T}}
$$

Here $A$ is the atomic mass, $N_{A}$ is Avogadro's number, $\rho$ is the density of the material, and the total scattering cross section is $\sigma_{T}=\sigma_{1}+\cdots+\sigma_{N}{ }^{43}$

Note that in the development of Eq. (2.2.4) the collision probability was assumed not to depend on particle energy (or time). This may seem to contradict the intuitive notion that the particle loses energy as it scatters through the media. The resolution is in the discrete characterization of the scattering process, as shown in Figure 2.2. In this type of Monte Carlo calculation, the particle is assumed to travel at constant energy between fixed scattering events, at which time the energy is demoted according to the type of scattering event which has been determined to occur.

Finally, the change in particle position after a scattering erent is found from $\mathbf{r}_{n+1}=$ $\mathbf{r}_{n}+s \Omega_{n}$. The explicit coordinate transformation equations between the co-moving "particle" coordinate reference frame (in which the physical scattering angles are computed) and the stationary "laboratory" reference frame (in which the host lattice is defined) are derived fully by Williamson and Duncan; ${ }^{44}$ we provide them here for reference:

$$
\begin{aligned}
\cos \theta_{n+1} & =\cos \theta_{n} \cos \theta+\sin \theta_{n} \sin \theta \cos \phi \\
\cos \left(\phi_{n+1}-\phi_{n}\right) & =\frac{\cos \theta-\cos \theta_{n+1} \cos \theta_{n}}{\sin \theta_{n} \sin \theta_{n+1}} \\
\sin \left(\phi_{n+1}-\phi_{n}\right) & =\sin \theta \sin \phi / \sin \theta_{n+1}
\end{aligned}
$$

The convention used in the above expressions is that the scattering angles in the laboratory frame for collision number $n$ are denoted by $\theta_{n}$ and $\phi_{n}$; the scattering angles in the particle frame are $\theta$ and $\phi$. Note also that the particle-frame polar angle $\theta$ will be computed from the application of a scattering mechanism, but that the azimuthal angle $\phi$ will be randomly

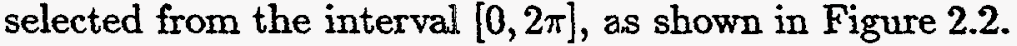

This process described above (which includes event selection, calculation of scattering information and trajectory computation) is repeated until some criteria for the termination of the particle track is met. This criteria is selectable by the user; this may include, for example, particle energy falling below a pre-established threshold, or particle ejection or backscattering from the solid. A flow diagram of this process is presented in Figure 2.1. 


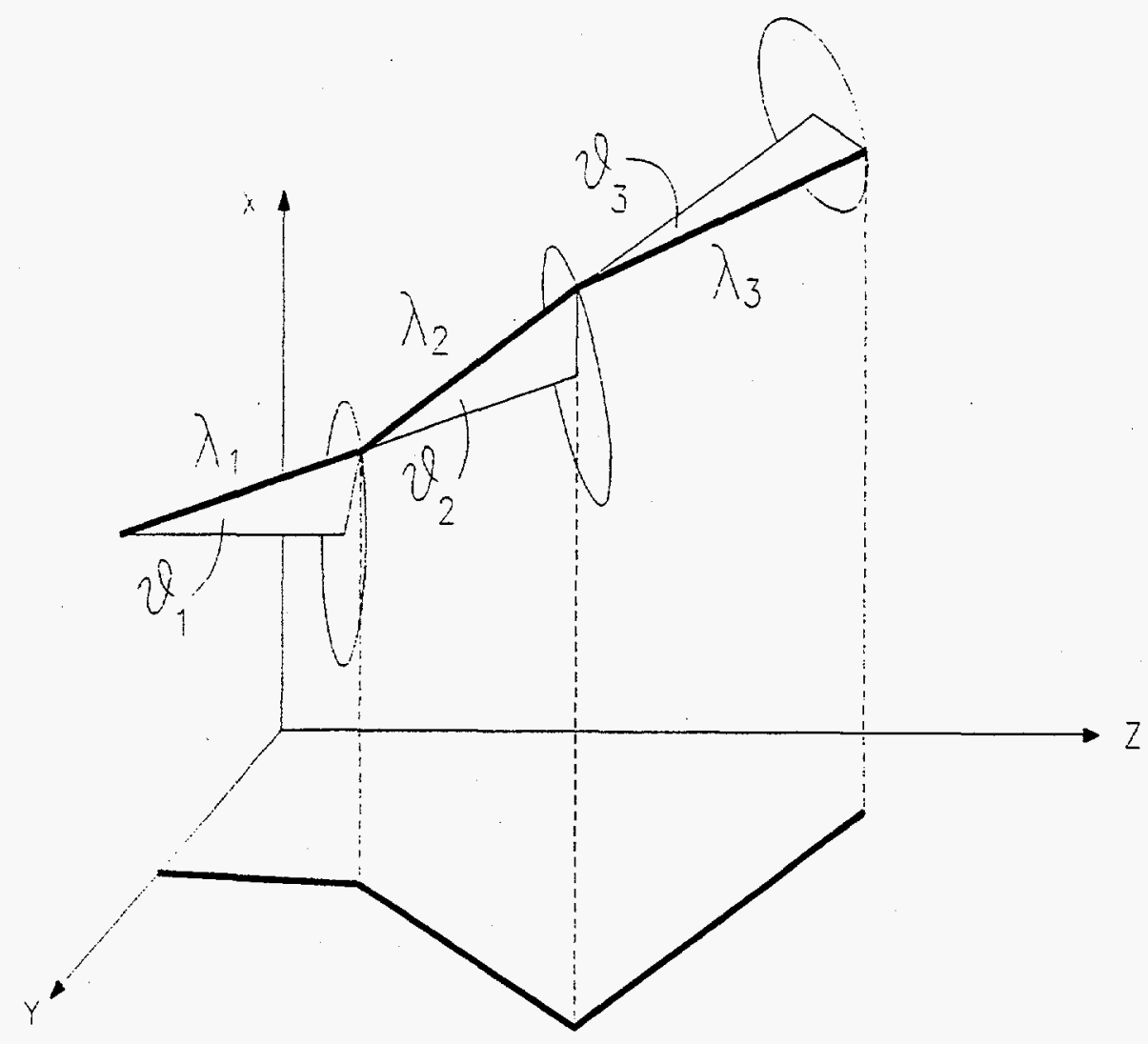

Figure 2.2 A schematic representation of a single particle "track" or scattering trajectory through the solid.

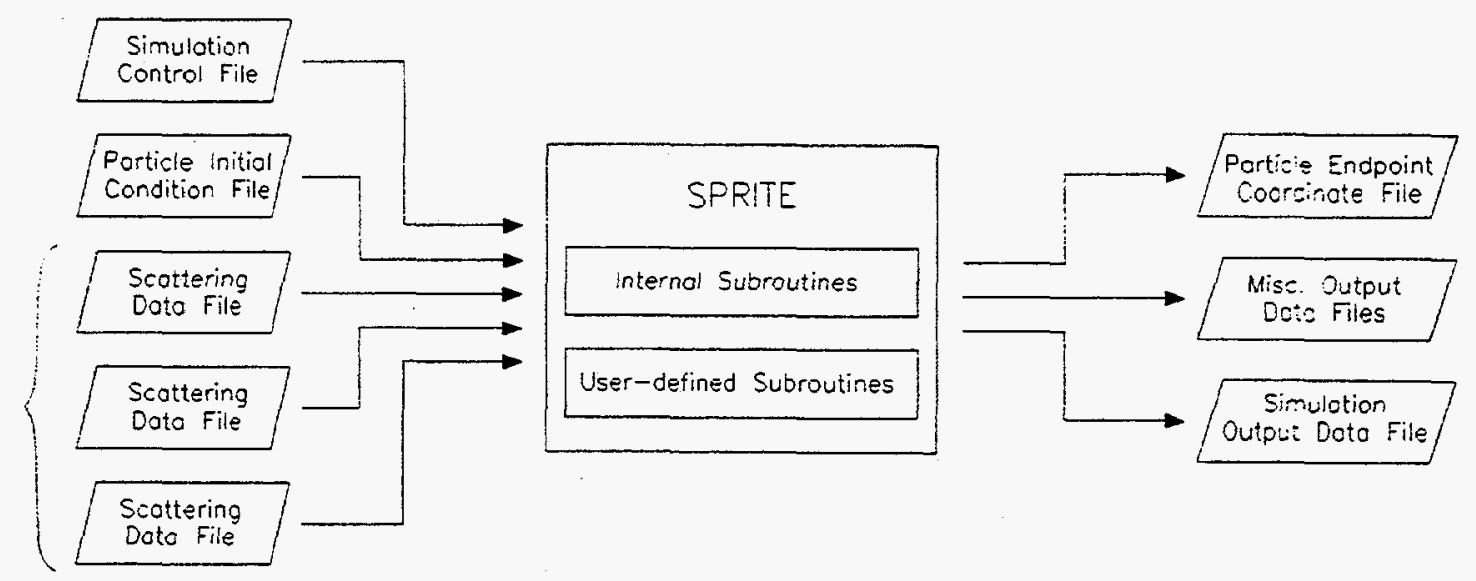

Figure 2.3 Block diagram of the required and optional files required in the SPRITE calculation. 


\subsection{User-Input Scattering Information}

Information about the particle transport calculation is supplied to SPRITE in the form of external datafiles created by the user. These include a simulation control data file (discussed below), a particle initial condition data file (discussed below), and data files which describe the physics of the particle-solid scattering (discussed in this section). Additionally, information about the scattering may be provided in the form of user-defined subroutines, which are appended to - and subsequently compiled with - SPRITE. The construction of such subroutines is discussed in the Appendix.

Two types of data files are recognized.- The first data file type, shown in Figure 2.4, handles the somewhat special case in which the the cross sections, energy loss per collision, and angular deflection per collision are known as unique functions of particle energy,

$$
\begin{aligned}
\lambda & =\lambda(E) \\
\Delta E & =\Delta E(E) \\
\Delta \theta & =\Delta \theta(E)
\end{aligned}
$$

SPRITE computes the necessary scattering information by first determining which two particle energies specified in this file (if any) bracket the current particle energy, then computes the required information by simple linear interpolation between these values. The interpolation scheme is discussed in greater detail below.

The second data file type, shown in Figure 2.5, handles the case in which the total scattering cross section and energy loss per collision are known as unique functions of particle energy, but in which the angular deflection is randomly sampled from the distribution formed by the differential scattering cross section. Recall the definition of the differential scattering cross section as the constant of proportionality between the number of incident particles $d n$ scattered into solid angle $d \Omega$ and the flux $F$,

$$
d n=F \frac{d \sigma(\theta, \phi)}{d \Omega} d \Omega
$$

From this the following physical interpretation is clear:

$$
\frac{d \sigma}{d \Omega} d \Omega=\frac{\text { number of particles scattered into } \mathrm{d} \Omega / \mathrm{sec}}{\text { number incident/sec/area on the incident plane }}
$$

Thus we can see that the differential scattering cross section, when properly normalized by the total cross section, is the probability distribution that a particle will scatter into a given solid angle.

SPRITE uses the total cross section information and energy loss per collision information in the same way as for the first data file type. However, the angular deflection per collision $\theta_{n}$ is sampled stochastically according to Eq. (2.3.1). Thus,

$$
\begin{aligned}
\lambda & =\lambda(E) \\
\Delta E & =\Delta E(E) \\
R & =\frac{2 \pi}{\sigma_{T}} \int_{0}^{\theta_{n}} \frac{d \sigma}{d \theta} \sin (\theta) d \theta
\end{aligned}
$$




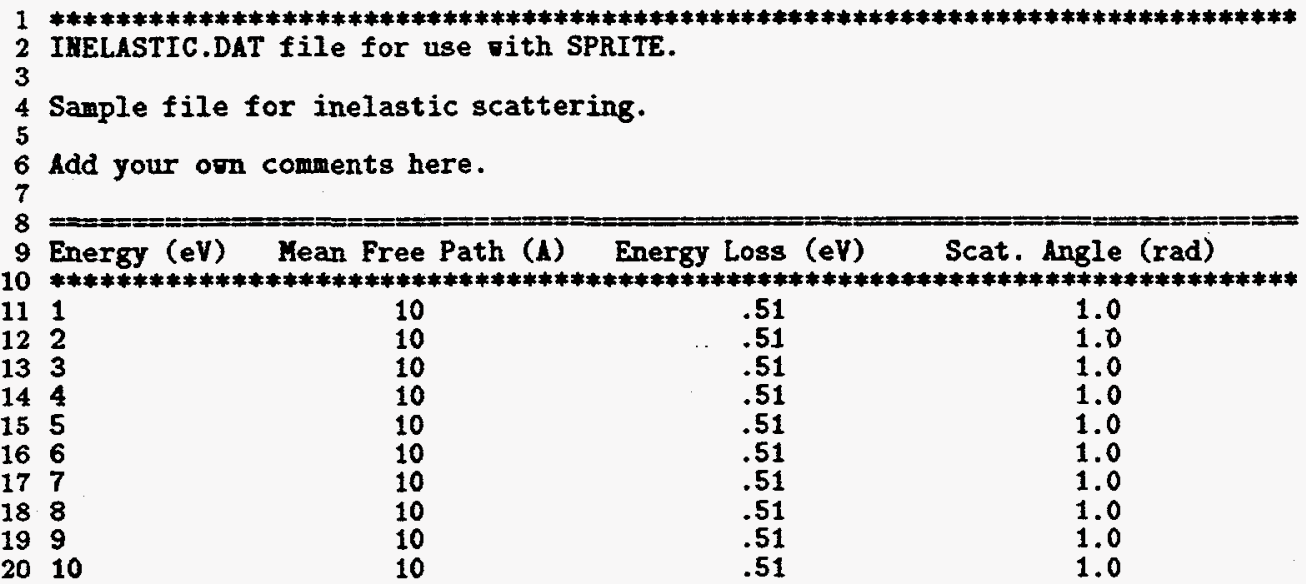

Figure 2.4 The first of two data file types which are recognized by SPRITE. The physical units corresponding to the numbers in the file may be arbitrarily specified by the user, provided all the data files are consistant.

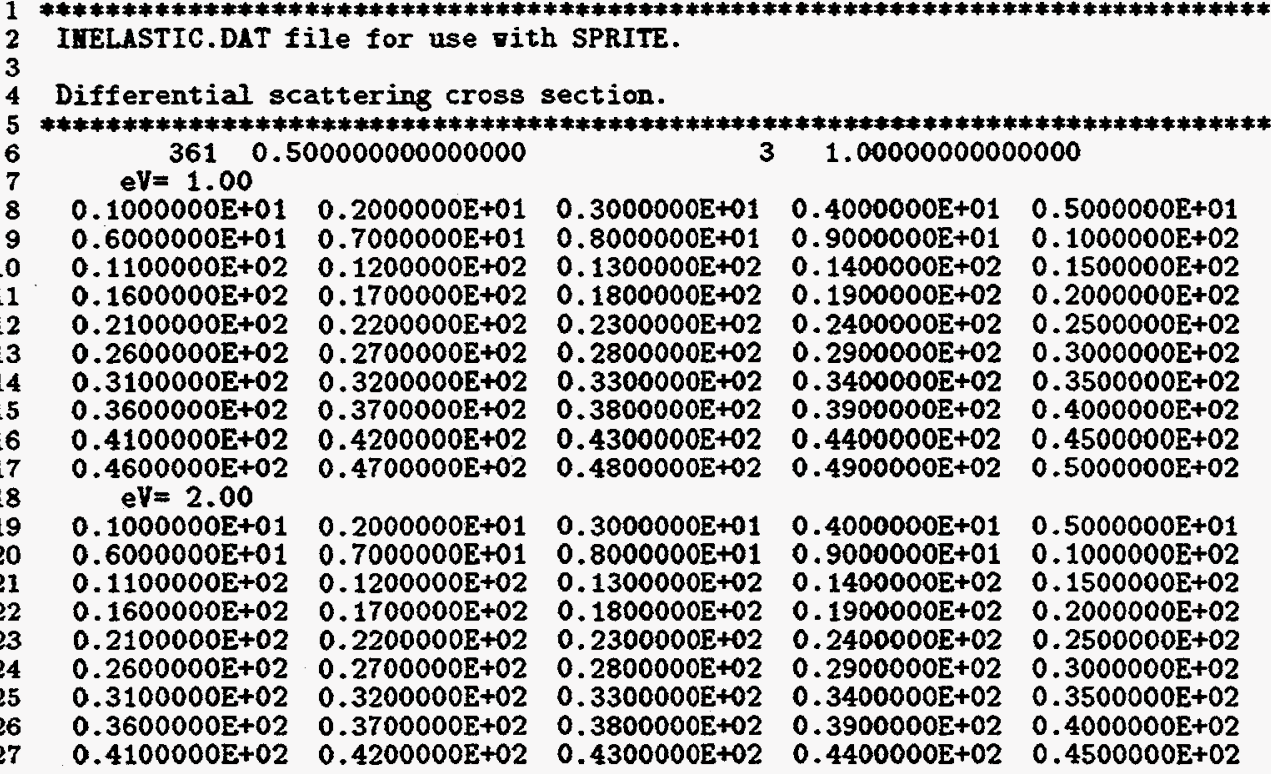

Figure 2.5 The second of two data file types which are recognized by SPRITE. This file contains the differential scattering cross section, according to which the angular deflection per collision is randomly sampled. 
where $R$ is a uniformly-distributed random number. Elastic scattering can be specified using either of these two file types, by simply specifying that the energy loss per collision is zero for each particle energy.

SPRITE implements simple linear interpolation to obtain information from the input data files, but it is made easy for the user to implement a more accurate interpolation scheme if desired. Interpolation in this manner has several advantages (including computational speed and reduced storage requirements), the most significant of which is the removal of any restrictions on the energy mesh used in the data files. For example, if the cross sections supplied by the user change dramatically in any energy range, the user can easily increase the accuracy of the calculation by supplying more data points in the energy range of interest, without regard to adherence to a strict mesh (linear/logarithmic/etc.).

A particularly simple scheme permits the user to specify the exact energy range over which a given scattering mechanism will be applied: Interpolation only (no extrapolation) is performed on the data files, thus the energy range defined in a data file is the range over which it will be applied in the sirnulation. For example, given the data files presented in Figures 2.4-2.5, the scattering mechanism represented by file 1 will be applied when the particle is in the energy range $1 \mathrm{eV}$ to $10 \mathrm{eV}$; that for file 2 will be applied in the range 1 $\mathrm{eV}$ to $2 \mathrm{eV}$.

In addition to these data files, information about scattering may be obtained from several subroutines which may be modified by the user and compiled with SPRITE. A subroutine in SPRITE (USER_INITIALIZE) is called once, at the start of the program; the user is free to include whatever code is necessary for initialization of the user-supplied routines. As the particle undergoes scattering events, information about the cross sections, energy loss per collision, and angular deflection per collision can be returned in the SPRITE subroutines USER_GETSIGS, USER_ENERGYLOSS, and USER_SCATANGLE. An example of the use of these user-definable subroutines is supplied in the program PSPRITE, the extension of SPRITE to the case of positron implantation. Information about modifying these subroutines is provided in the Appendix.

\subsection{Initial Conditions}

Important control parameters for the simulation are contained in a simulation control file. Information about the initial state of the particles is contained in a particle initial-condition file. These files have been constructed to provide as simple an interface to the transport calculation as possible. In general, the user is expected to construct and store a number of these files relevant to the systems of interest. For example, once a file is constructed which defines a solid (such as a semi-infinite aluminum sample) or the initial state of the particles (such as all particles normally incident with $10 \mathrm{keV}$ kinetic energy), this file can be used repeatedly. We discuss the information contained in these fles below.

Note that it is also possible to specify a functional form for the initial conditions, rather than by obtaining them from a file. For this, however, it is necessary to incorporate the function into the SPRITE code, and recompile the code. This procedure is discussed in the Appendices.

\subsubsection{Description of the Solid}




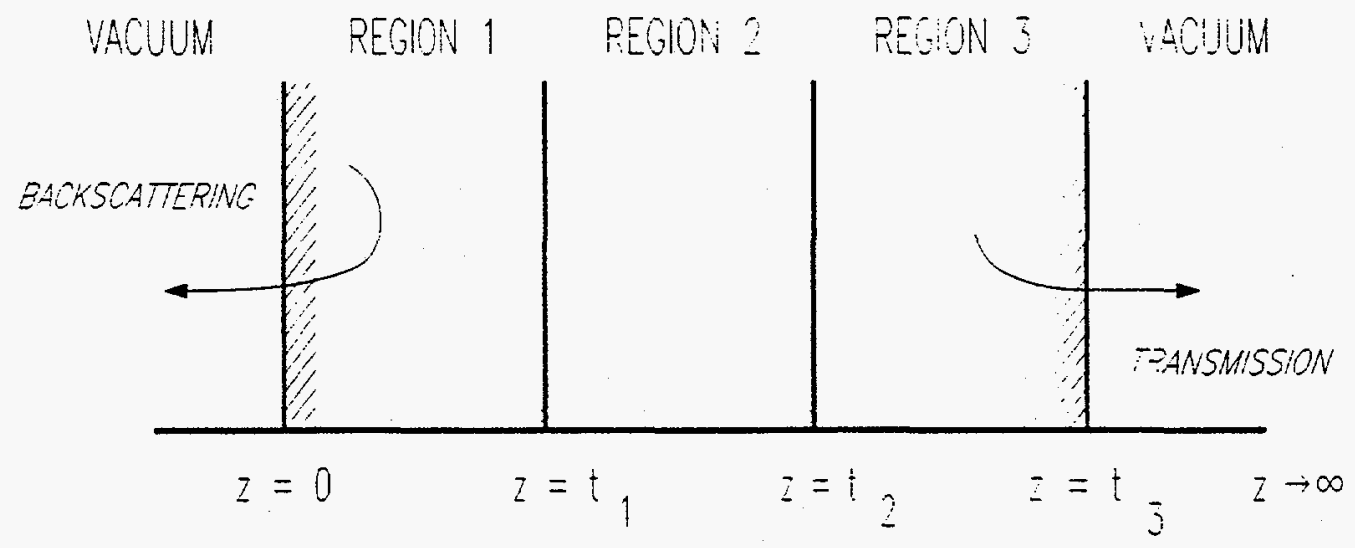

Figure 2.6 A schematic representation of the convention used to describe the multilayers which compose the solid. This example depicts a multilayer composed of three "slabs."

In this section we address the information contained in the simulation control file. This file contains information about the following topics: the physical description of the solid, including spatial extent and composition; specification of the scattering mechanisms, such as the names of the external data files which contain scattering information, or an indication that a user-incorporated subroutine is to be invoked; information about interface effects, such as values of the work function between two adjoining layers in a multilayer sample; and additional information, such as whether to invoke a user-friendly "verbose" mode, in which information about the scattering calculations is to be displayed on the terminal as it is computed. Each of these topics is discussed in greater detail below.

Description of the solid. Examples of simulation control files are presented in Figures 2.7-2.8. The first functional record of the control file specifies the number of layers in the multilayer system. The file structure thereafter depends on whether the system contains one layer or more than one layer, as shown in Figures 2.7-2.8. For each layer, additional information must be specified. The material name entry is provided for the convenience of the user; the calculation does not use this information. The thickness of the layer must be given; in the case of a semi-infinite solid, it is sufficient to supply a suitably large thickness such that no particles will be expected to reach the inner boundary. The atomic mass of the solid and the mass density are specified, so that the mean free paths can be computed according to Eq. (2.2.6). Physical information about the solid (as well as all other material-dependent information provided for the calculation) can be provided in any dimensions or units, provided the units are used consistantly throughout the calculation. This is discussed in a separate section below.

The conventions used to describe the solid are depicted in Figure 2.6. The $N$-th layer is assumed to begin at $z=a$ and to extend a depth of $z=a+t_{N}$, where $t_{N}$ is the thickness of the $N$-th layer, specified in the control file, and

$$
a=\sum_{i}^{N-1} t_{i}
$$

The term backscattering is used to denote a particle crossing a layer boundary in the 
direction of decreasing $z$-coordinate (i.e., from right to left in Figure 2.6. The term transmission is used to denote a particle crossing a layer boundary in the direction of increasing $z$-coordinate (i.e., from left to right in Figure 2.6). The SPRITE calculation records statistical information about particles which are backscattered or transmitted; this is discussed in a separate section below.

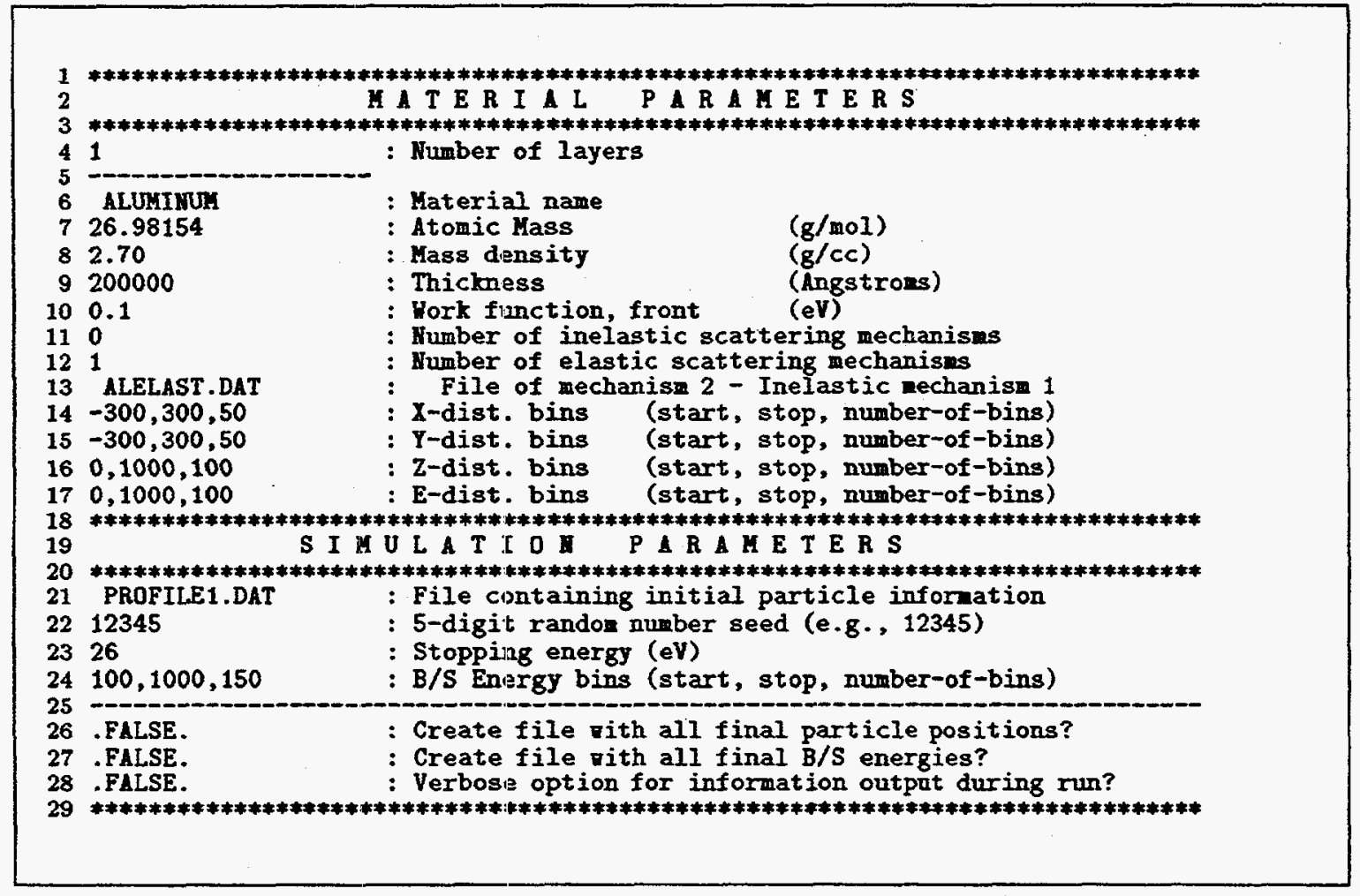

Figure 2.7 The main control file recognized by SPRITE. This file contains the control parameters for the simulation. This example defines a single solid.

Specification of scattering mechanisms. Information about what scattering mechanisms to invoke must be provided for each layer in the system. In this way, the user is free to completely define when each scattering process will be considered. It is of course possible to mandate that the same scattering mechanism be applied to each layer in a multilayer system (simply by duplicating the specified file names). Note that the SPRITE calculation will apply the scattering mechanisms only over the kinetic energy range specified in the scattering files, as discussed above.

Interface effects. If the particle is determined to have crossed an interface, either between two layers or between the first layer and vacuum, a subroutine in the SPRITE program (INTERFACE_ENCOUNTER) can alter the trajectory, if desired. Currently this subroutine will evaluate the particle energy and specularly reflect the particle if the normal 


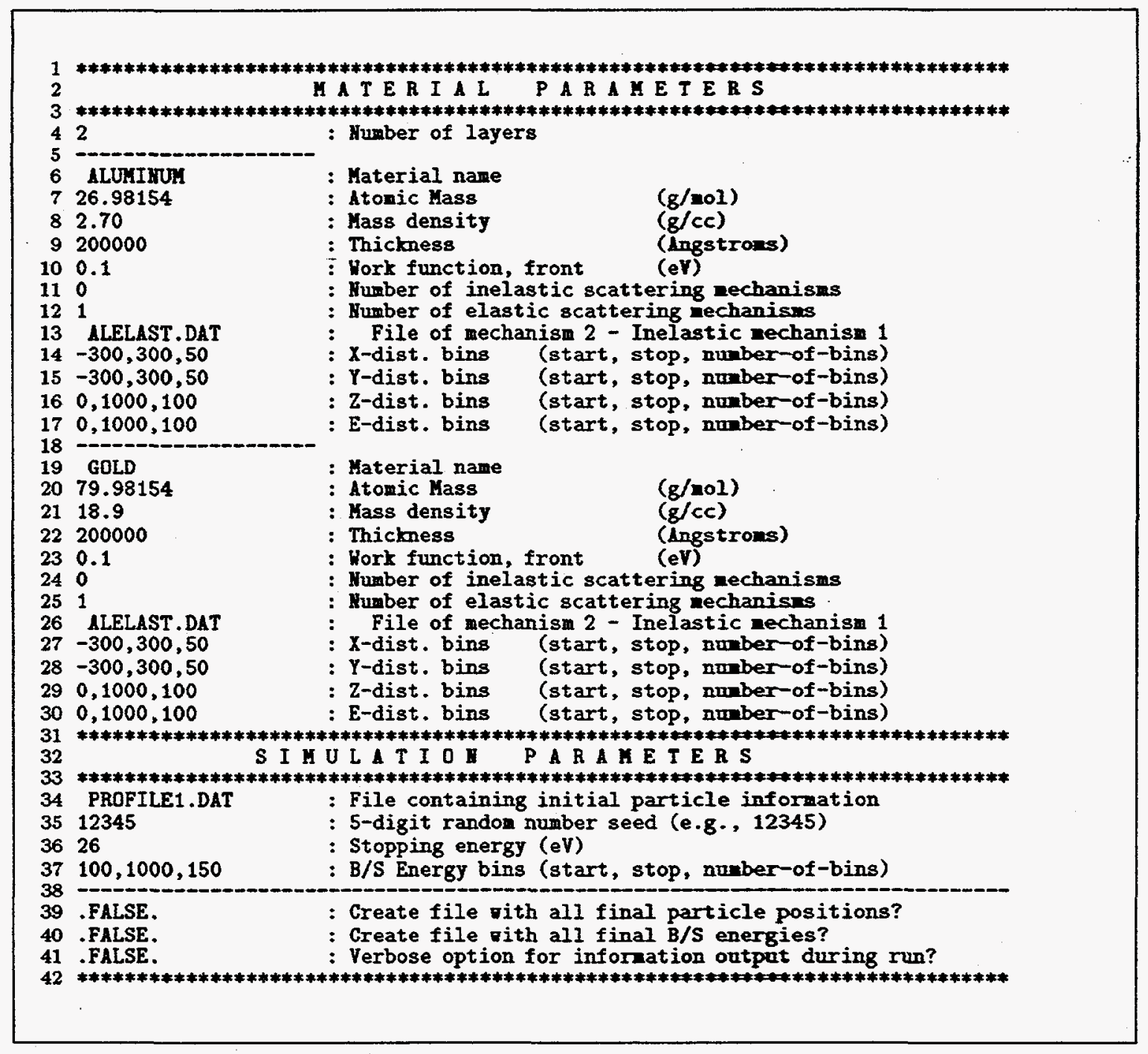

Figure 2.8 The main control file recognized by SPRITE. This file contains the control parameters for the simulation. This example defines a multilayer.

component of the kinetic energy is less than the work function difference at the interface; this subroutine will also reflect a fraction of incident particles at all energies, determined by the quantum-mechanical reflection coefficient for particles incident on a squary energy-step barrier. However, the INTERFACE_ENCOUNTER subroutine is easily modifiable by the user. Possible modifications include more complicated energy- and angle-dependent reflection, or simple transmission at all energies across the interface (either by specifying a zero work function, or by simply commenting out the call to INTERFACE_ENCOUNTER).

Output control information. Also contained in the simulation control file is information regarding how the output of the SPRITE calculation should be prepared. This includes 


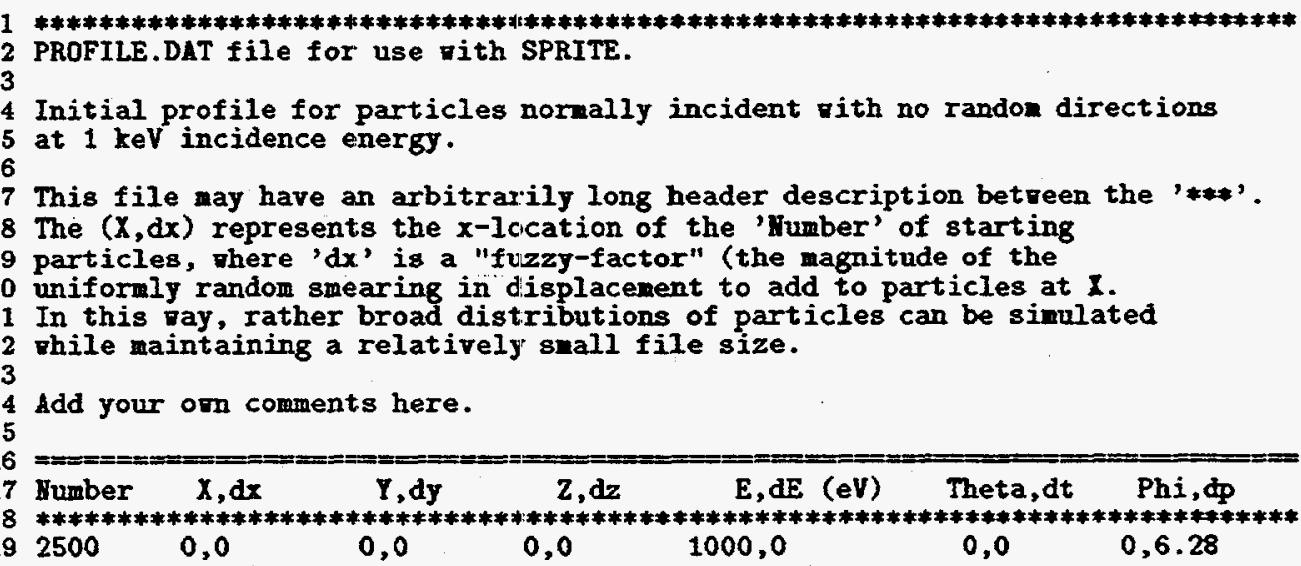

Figure 2.9 The file containing the initial conditions for the simulation. The file may contain arbitrarily many records which specify the initial distribution of particles.

information about the spatial and energy meshes upon which the particle energies (such as the energy upon backscattering or transmission) and trajectory endpoint positions will be binned. The actual distributions are recorded as output, as well as basic statistical information, such as the mean value and first few moments of the distribution. This output is discussed in greater detail below.

Miscellaneous information. Finally, several miscellaneous features of SPRITE can be specified in the simulation control file. These are discussed below.

- Verbose Option. Normally the SPRITE calculation is "silent" - that is, it does not display information on the terminal, except possibly for brief updates regarding the proximity in time of the calculation to completion. The SPRITE calculation can be performed in an optional "verbose" mode, in which information about the calculation (such as the values of the cross sections, particle position, etc.) is continually written to the terminal (standard output device) as it is computed. This feature can be quite useful for debugging purposes. An example of this verbose output is provided in Figure 2.10.

- Particle Position File. The SPRITE calculation can produce, as an optional feature, a file containing the endpoint coordinates of all the particles in the simulation. In general this file may be quite large, and thus it is expected that such a file will not be created by default.

- B/S Energy File. The SPRITE; calculation can also produce, as an optional feature, a 


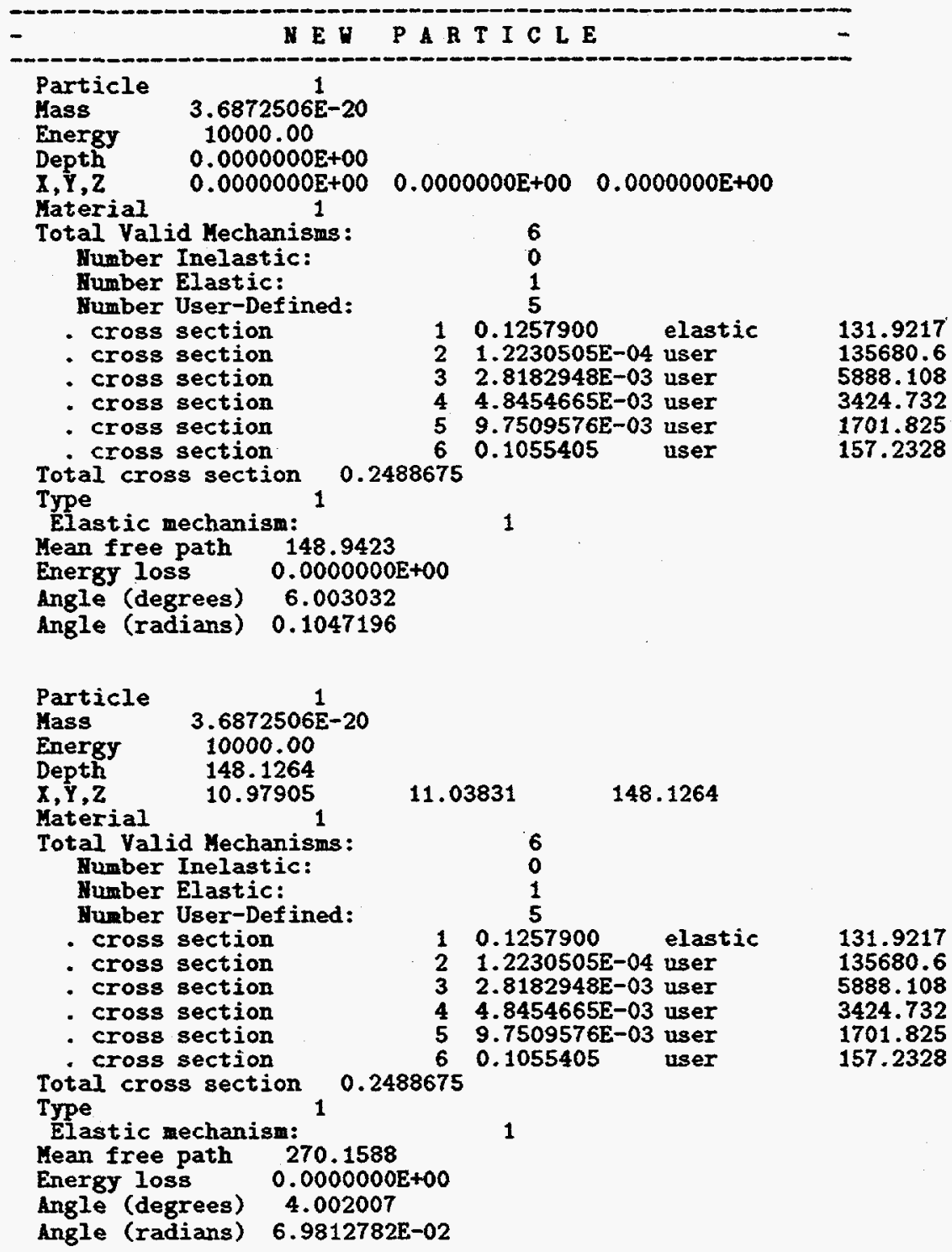

(1)

1

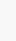


The initial conditions required by the calculation are the initial spatial and momentum distribution of the particles. These are contained in an external data file. An example of this file is provided in Figure 2.9. This file is structured for maximum convenience of use. In the case in which all particles begin with the same momentum at the same location in the material, only one record in this file is required, as shown in Figure 2.9. Multiple records can be given to specify a complete initial distribution. The "fuzziness" factors supplied in the file represent the degree of noise introduced which is randomly introduced into energy and momentum. For example, when the energy $(E, \delta e)$ is specified, the initial particle energy is computed according to

$$
\text { TRAJECTORY ENERGY }=\mathrm{E}+\mathrm{DE} *(2 * \text { RANHUM }-1)
$$

In this way, broad distributions may be accurately represented with relatively small files. Additionally, this method of specification makes it possible to begin the simulation with a completely random directional distribution, via $(\theta=0, \delta \theta=\pi)$; this could be of use, for example, in a calculation involving an initial point-source of particles.

\subsection{Physical Dimensions and Units}

The only physical units default-coded into SPRITE are radians, so that geometric calculations involving user-supplied angles may be performed directly with Fortran intrinsic functions. For maximum ease of use in a wide variety of problems, it will be assumed that the input files required by SPRITE will use a consistant and user-defined set of units. For example, assume the scattering information file shown in Figure 2.4 has particle energy, cross section, energy loss per collision, and angular deflection per collision in units of $\mathrm{eV}, \AA^{2}, \mathrm{eV}$, and radians, respectively. Then the initial particle information shown in Figure 2.10 represents depth in $\AA$ and energy in eV. Similarly, the binning information contained in SPRITE.DAT must be in $\AA$ for the stopping profile, and $\mathrm{eV}$ for the energy distributions. SPRITE converts the scattering cross sections to mean free paths by multiplication by a conversion factor, defined in Eq. (2.2.6). This factor is computed using the material density and atomic number, as specified by the user in the simulation control data file. An example of the factor used in converting cross section to mean free path is obtained as follows: Assuming the material is aluminum (Al) with a density of $2.7 \mathrm{~g} / \mathrm{cm}^{3}$ and an atomic mass of $26.98 \mathrm{~g} / \mathrm{mol}$, the conversion factor is

$$
\text { MATERIAL_MFP2SIG }=\frac{26.98 \mathrm{~g} / \mathrm{mol}}{6.022 \times 10^{23} \mathrm{~mol}^{-1} \times 2.7 \mathrm{~g} / \mathrm{cm}^{3}}
$$

\subsection{Output Information}

As discussed above and as depicted in Figure 2.3, the SPRITE program requires two files (a simulation control file and a particle initial condition file), plus possible additional. files containing user-specified information about the scattering mechanisms. The SPRITE program performs then performs the transport calculation, and appends the results of the calculation to the simulation control file. It is expected in general that a small number of particle initial condition files will be required; a set of initial conditions is usually 
determined only once for the system of interest (for example, a user may be interested in particles normally incident on the surface of a solid, etc.). However, the user is apt to have a large number of simulation control files. The calculation results are appended to the control file in order to greatly simplify data management and analysis. In this way the user can store the results of the calculation together with the simulation parameters which led to those results.

The results generated by SPRITE fall into two broad categories: statistical information about the scattering process, such as the frequency distribution of the selected scattering events; and physical information, such as the endpoint distribution of the particles, mean scattering times, etc. These are discussed in the following sections.

Statistical information. SPRITE records the frequency distribution of the scattering events which occured. These distributions are reported for each layer in the system, and for each of the three possible types of scattering mechanisms (the two file types, and a possible user-defined subroutine). SPRITE also records the total number of scattering events. The user can control the output of statistical information (e.g., number of bins, bin sizes, etc.) by indicating the desired choices directly in the simulation control file, as shown in Figure 2.7.

Physical information. SPRITE records a large amount physical information about the scattering process. This information includes the following:

- Endpoint trajectory distribution. The coordinates of the endpoint locations of the particles are binned in a histogram, the definition of which (starting and final positions, and number of bins) is specified in the simulation control file by the user. Let $Z_{1}$ and $Z_{N}$ denote the initial and final depths specified by the user for the mesh (this procedure is also performed for $X$ and $Y$, the meshes for which the user must provide separately), and let $N$ denote the number of depth bins requested. Then an $N$-bin array is output following the calculation, where the $i$-th element $Z_{i}$ contains the number of instances in which the endpoint $z$-coordinate of the particles resided in space between $Z_{1}+i \delta$ and $Z_{1}+(i+1) \delta$, where $\delta$, the mesh size, is equal to $\left(Z_{N}-Z_{1}\right) / N$. In addition to the histogram, the first several moments of the distribution are calculated and reported in the output file. It is important to note that, because the mesh over which the coordinate information is binned is specified by the user, not every particle which stops in the host lattice will necessarily be binned in these histograms; in this way, the user is free to examine specific endpoint regimes via arbitrary specification of the meshes.

- Energy distributions, backscattered and transmission. As with the depth coordinates discussed above, the energy distribution of the particles backscattered from a given layer, as well as those transmitted though a given layer, are binned as discussed above.

- Mean ensemble information. SPRITE reports basic information about the total ensemble of particles simulated. This information includes the mean time required for the particles to reach the endpoint energy of the simulation (i.e., thermalization time), and the fraction of particles backscattered from and transmitted through the layers. The thermalization time is computed according to a simple linear approximation, 


$$
\tau=\sum_{i=0}^{N} \tau_{i}
$$

where $\tau_{i}$ is the time between the $i-1$ and $i$-th scattering event, and the sum is over all $N$ scattering events. The particle energies and particle mass (specified in the simulation control file) are used to compute $\tau_{i}$ via

$$
\tau_{i}=\frac{\lambda_{i}}{\sqrt{2 E_{i} / m}}
$$

Note that it is trivial to incorporate a more sophisticated time calculation routine, should the user so desire. More information regarding this is presented in Appendix. D. 


\section{Examples of SPRITE Data}

\subsection{Overview}

SPRITE was designed to be flexible, easy-to-use and easy-to-modify. Thus the type and amount of data which can be readily extracted from this calculation is almost limitless. In this section we provide a number of examples of the type of data which can be generated, either directly or else with very minor modification to the programs.

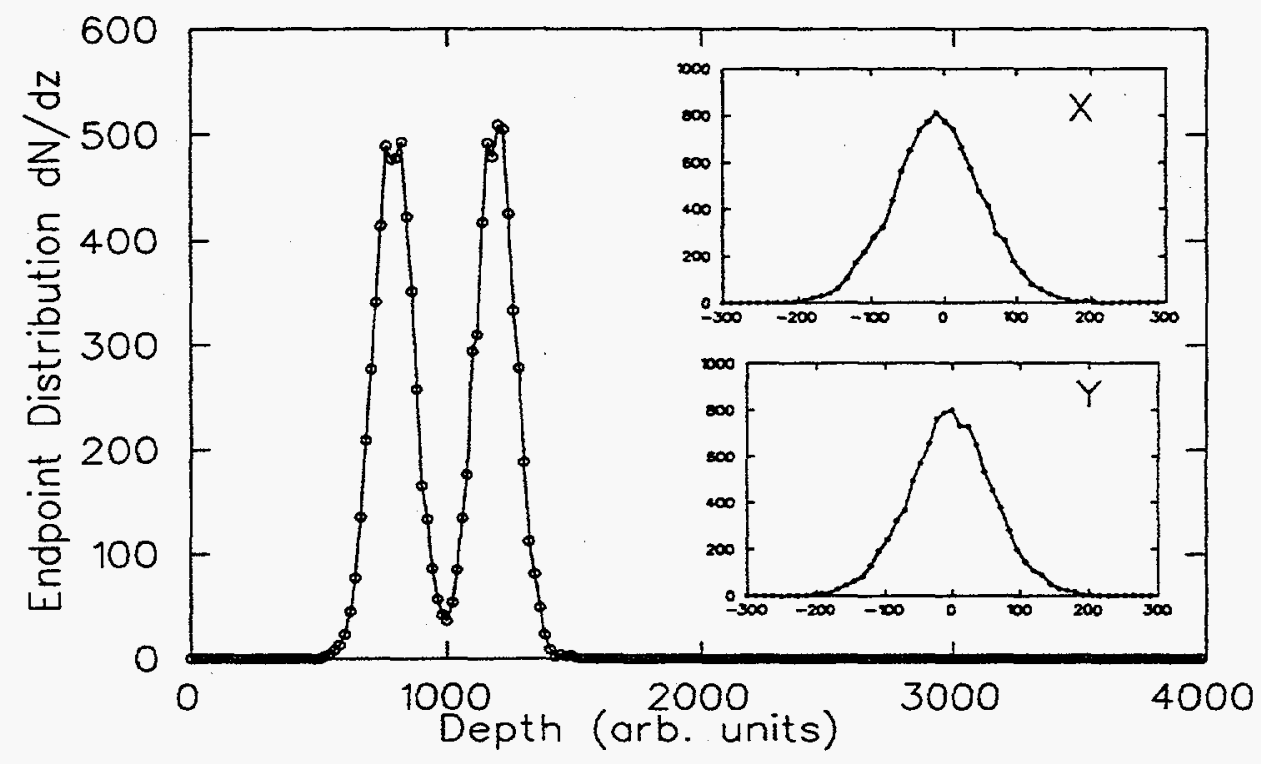

Figure 3.1 The distribution in $x, y$, and $z$ of the endpoint coordinates of particles scattering from two point sources in a homogeneous solid. 


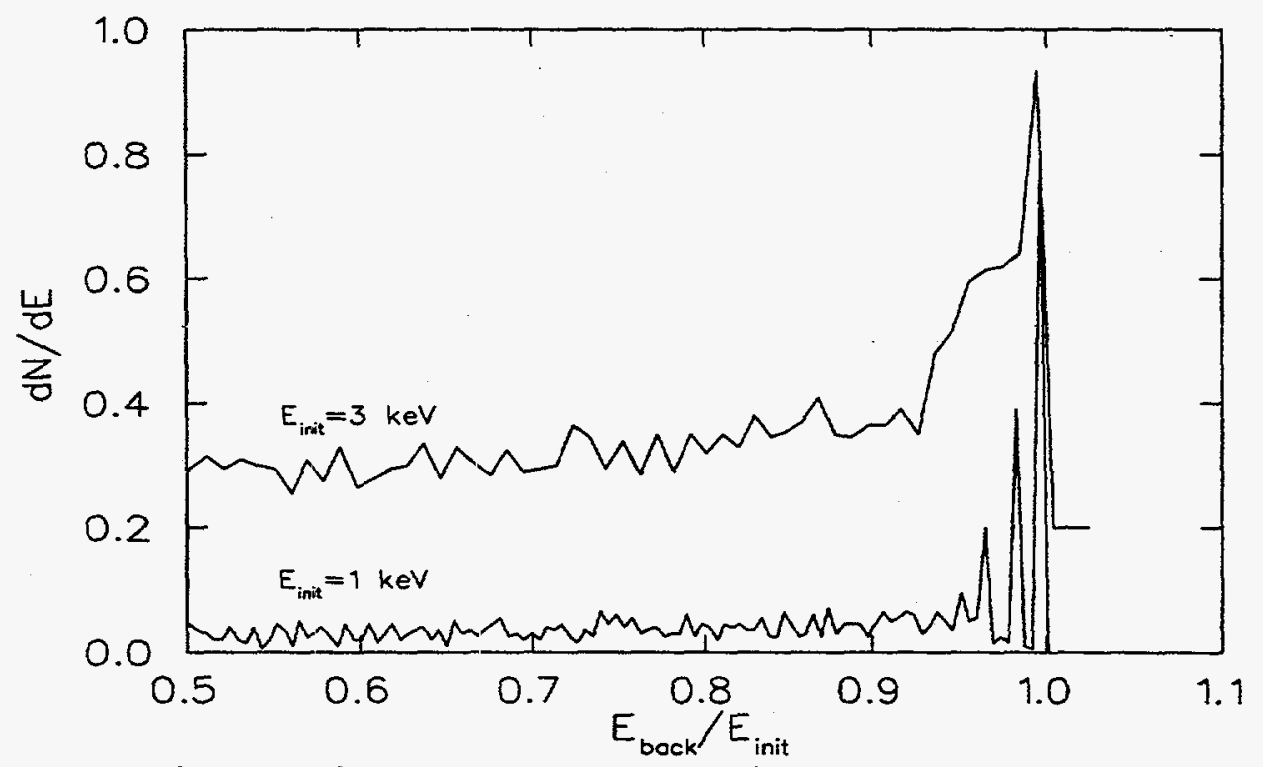

Figure 3.2 The energy distributions of positrons backscattered after normal implantation in Al. The series of peaks observable at $1 \mathrm{keV}$ incident energy are due to a discrete plasmon energy loss process incorporated into the simulation.

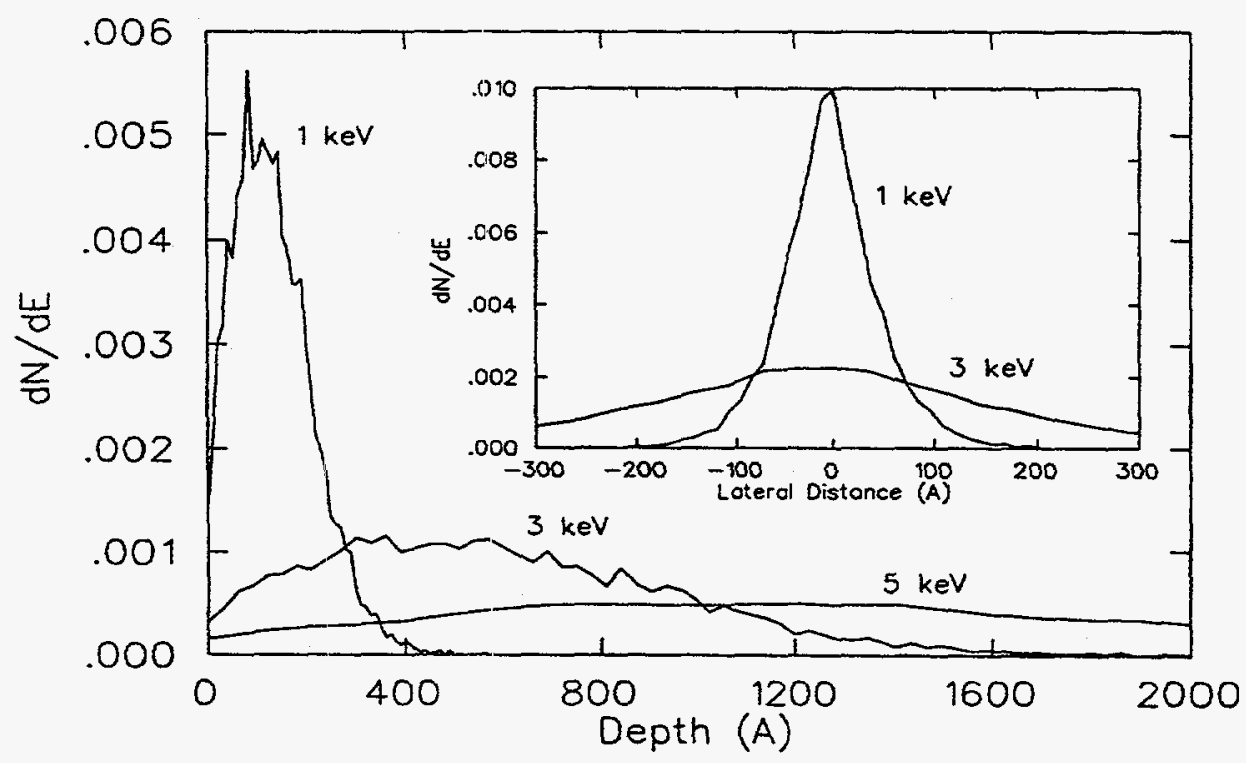

Figure 3.3 Lateral and depth stopping profiles of particles normally incident on $\mathrm{Al}$, for several incident energies. 


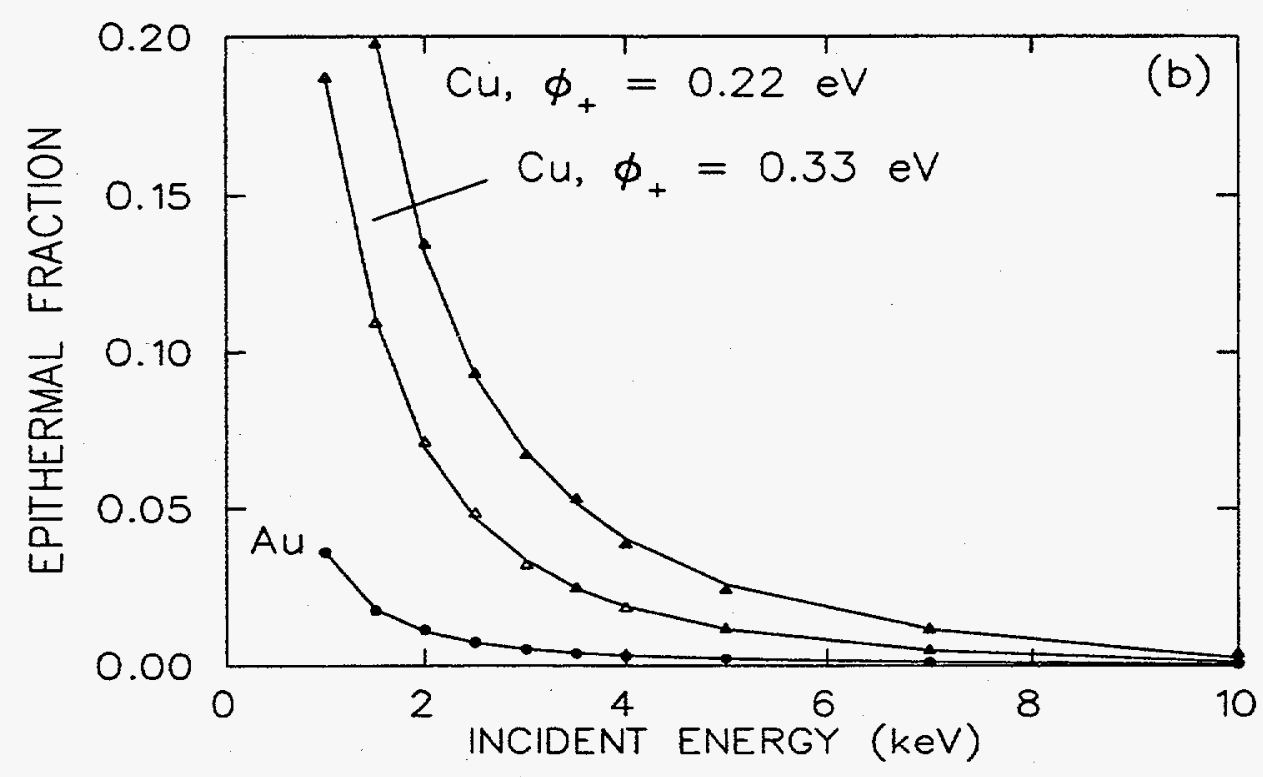

Figure 3.4 The fraction of particles which, after implantation into several materials, overcome the surface energy barrier and are re-emitted.

$$
a^{\prime} \cdot
$$

FRACTION

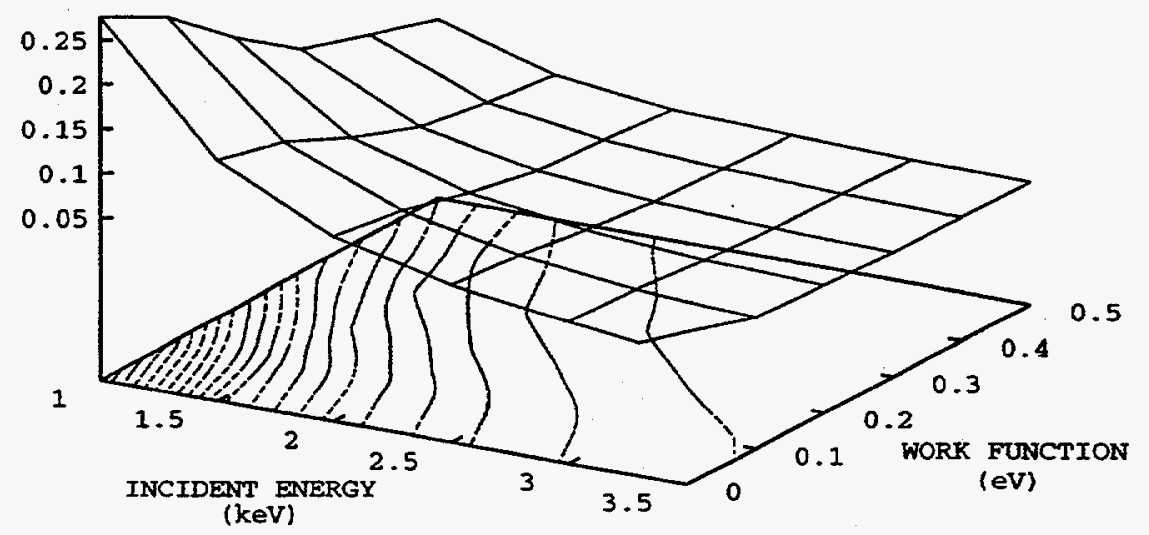

Figure 3.5 The fraction of particles re-emitted after implantation in a metal, shown as functions of both incident energy and surface work function. 


\section{POS-SPRITE}

\subsection{Overview}

The POS-SPRITE family of programs is comprised of the following programs:

- PSPRITE, a modified version of SPRITE, containing a subroutine to provide information about inelastic positron- or electron-solid scattering

- PATOMIC, an ab initio calculation of atomic potentials using a relativistic DiracHartree-Slater-Fock formalism

- PVCALC, a program to renormalize the atomic potentials to account approximately for the effects of the host lattice

- PSIGEL, a partial wave calculation of elastic positron- or electron-atom differential and total scattering cross sections

- POSRPA, a calculation using the random phase approximation of the valence-level differential scattering cross section

PSPRITE is a version of SPRITE which contains a user-defined subroutine which returns information relevant to electron/positron-solid interactions. The calculation of the single particle trajectories proceeds for PSPRITE in the same general way as for SPRITE. Information about elastic scattering is provided by means of external data files, while information about inelastic scattering is obtained though the subroutine attached to the program. Initially, the cross sections for all available elastic and inelastic mechanisms are computed, and a specific scattering mechanism is randomly selected according to the probability distribution, Eq. (2.2.1), discussed in Section 2. Once the scattering type is determined, the mean free path is calculated from Eq. (2.2.5). The energy loss and scattering angle corresponding to this scattering type are then computed, using formalism discussed in the next section.

The final choice of scattering mechanisms is the central decision in calculations such as the present one. There is no general consensus regarding the "best" choice of scattering mechanisms to use. Some mechanisms, such as the Penn description of the mean free path, are based on experimental data obtained in a limited energy regime; other scattering formalism, such as the optical scattering model developed by Penn and co-workers, does not readily distinguish between positron and electron scattering, and thus important differences may not be well accounted for in all energy regimes or circumstances. Thus the validty of applying some mechanisms to certain materials or over certain energy ranges is not completely clear. Unfortunately, experimental work involving positrons is far less complete than for electrons, and thus we are forced to rely upon a certain degree of faith.

To partially mitigate this problem, the present work incorporates several different scattering mechanisms, and it is possible for the user to explore the differences which result when the various mechanisms are applied. In this way, the user is not restricted to one result, but rather can obtain a range of results, defined by various theoretical approaches. 


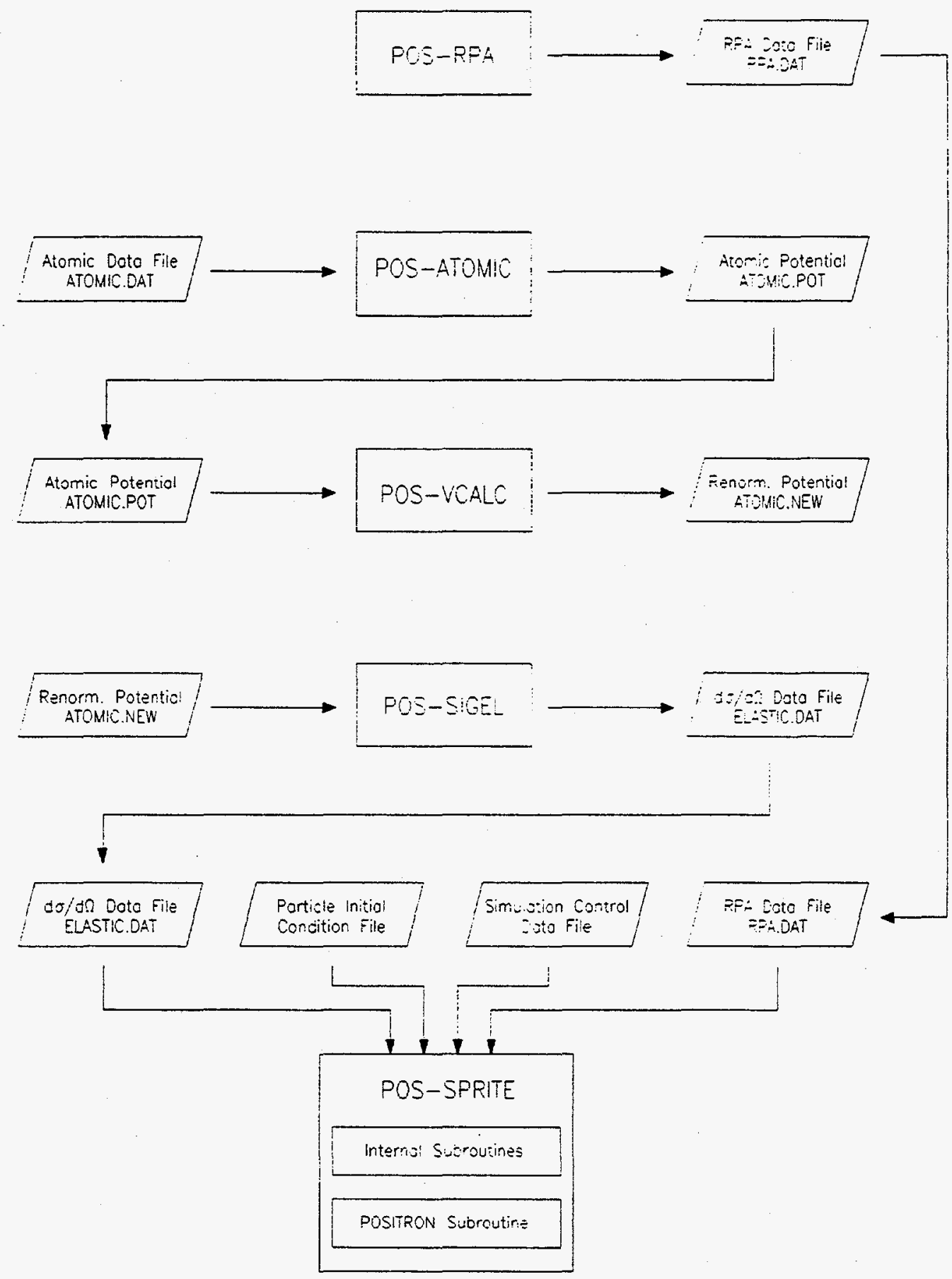

Figure 4.1 Block diagram of the required files and ancilliary programs associated with the PSPRITE calculation. 
Further, owing to the extensibilty of SPRITE, the transport engine, new mechanisms are easily incorporated into the calculation.

We do note that, aside from some freedom in choosing which scattering mechanisms can be applied, this calculation contains no adjustable or arbitrary parameters. Constants which appear in the mathematical formalism represent the best available experiment or theoretical data. Further, the user has the freedom to replace many of the constants (such as electron binding energies, plasmon frequencies, etc.), when new experimental data becomes available, or in order to test the effect these material-specific constants have on the final results of the calculation.

In the next two sections we address the elastic and inelastic scattering mechanisms currently packaged with POS-SPRITE. These mechanisms have been found to give reasonable agreement with available experimental data; full details and benchmark comparisons with data will be presented elsewhere. A reference chart of the mechanisms employed appears in Table 4.1..$^{-12}$

\begin{tabular}{llll} 
Electronic Level & Mean Free Path & Energy Loss & Scattering Angle \\
\hline Core (K-Shell) & $\begin{array}{l}\text { Kolbensvedt } \\
\text { or Gryzinski }\end{array}$ & $\begin{array}{l}\text { Rohrlich/ } \\
\text { Carlson }\end{array}$ & $\begin{array}{l}\text { binary collision } \\
\text { approximation }\end{array}$ \\
Core (non-K-shell) & Gryzinski & $\begin{array}{l}\text { Rohrlich/ } \\
\text { Carlson }\end{array}$ & $\begin{array}{l}\text { binary } \\
\text { approximation }\end{array}$ \\
Valence (plasmon) & Penn & $\begin{array}{l}\text { Quinn } \\
\text { Oliva }\end{array}$ & RPA \\
Valence (quasiparticle) & Penn & $\begin{array}{l}\text { Quinn } \\
\text { Oliva }\end{array}$ & RPA
\end{tabular}

Table 4.1 A schematic representation of a the scattering mechanisms incorporated into PSPRITE. Each mechanism is discussed in detail in the text. 


\subsection{Elastic Scattering}

Elastic scattering of an implanted positron or electron by the atomic cores of the host lattice is an important mechanism and has a significant effect on the final depth distribution of positrons in the lattice. Information about elastic scattering is provided to PSPRITE via external data files, as discussed in the previous section. The information required is the total scattering cross section (TCS) and the differential scattering cross section (DSCS), the latter being used to determine the scattering angles. The user is free to supply elastic scattering data files, constructed using experimental or theoretical methods of the user's own choosing; or else the user may employ ancilliary programs supplied with POS-SPRITE for this purpose: PATOMIC, PVCALC, and PSIGEL. The calculation scheme and these programs are discussed in some detail here; a series of tutorials, together with sample results, are packaged with the programs in the source code distribution.

Elastic scattering cross section. As an approximation to computing the complicated dynamical trajectory of a charged particle within a crystal, the incident positron is assumed to undergo elastic scattering from a single atom potential (discussed below) which has been modified to account for the effects of the lattice. Using the method of partial waves, the differential scattering cross section for scattering from a central potential is given by

$$
\frac{d \sigma_{e l}}{d \Omega}=\frac{1}{k^{2}}\left|\sum_{l=0}^{\infty}(2 l+1) e^{i \delta_{l}} \sin \delta_{l} P_{l}(\cos \theta)\right|^{2}
$$

where $P_{l}$ is the $l^{\text {th }}$ Legendre polynomial, $k$ is the wave number, and $\delta_{l}$ is the phase shift of the $l^{\text {th }}$ partial wave. To obtain the phase shifts, the radial Schödinger equation

$$
\left[-\frac{d^{2}}{d r^{2}}+2 V(r)+\frac{l(l+1)}{r^{2}}-k^{2}\right] u_{k, l}=0
$$

where $V$ is the atomic potential (discussed below), is solved numerically. Several methods of solving the radial Schrödinger equation were tested during the development of this calculation, including a Numerov method ${ }^{45}$ and Milne's method. ${ }^{46}$ The latter technique was found to give the most stable solutions and thus was incorporated into the calculation (PSIGEL), as discussed below.

By matching the solution computed in this way to the free-particle solution at the Wigner-Seitz radius, $r_{w s}$, the phase shifts can be computed from

$$
\tan \delta_{l}=\frac{k j_{l}^{\prime}\left(k r_{r s}\right)-\gamma_{l} j_{l}\left(k r_{w s}\right)}{k n_{l}^{\prime}\left(k r_{r s}\right)-\gamma_{l} n_{l}\left(k r_{w s}\right)}
$$

where $\gamma_{l}$ is the ratio of the slope to the value of the radial wavefunction, and $j_{l}$ and $n_{l}$ are the spherical Bessel and Neumann functions, respectively. We have found it practical to consider as many as one hundred partial waves, and thus some care has been taken to ensure that the method of computing these waves is an accurate one. The routines for evaluating the Bessel and Neumann functions were rigorously tested, and comparisons 
were made with published tables. ${ }^{46}$ The calculation employs double-precision arithmetic, using forward recursion methods for the regular spherical Bessel functions and backward recursion methods for the Neumann functions.

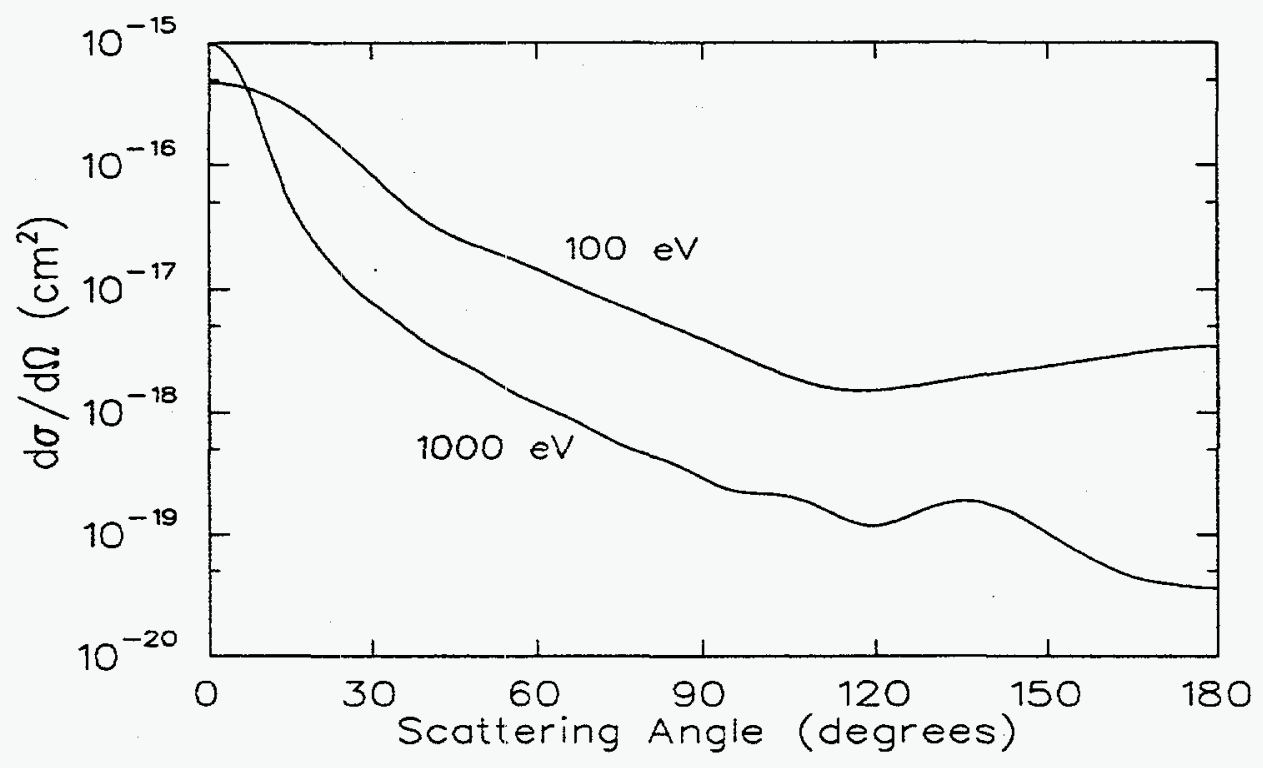

Figure 4.2 The differential scattering cross section for aluminum $(\mathrm{Z}=13)$ for incident positrons, calculated at two energies by PSIGEL.

Once the phase shifts and the differential cross sections are known, the total cross section $\sigma_{e l}$ may be calculated, either by direct numerical integration

$$
\sigma_{e l}=\int\left(\frac{d \sigma}{d \Omega}\right) d \Omega
$$

or by using the partial wave result ${ }^{47}$

$$
\sigma_{e l}=\frac{4 \pi}{k^{2}} \sum_{l=0}^{\infty}(2 l+1) \sin ^{2} \delta_{l}
$$

As shown in the inset of Figure 4.3, the results obtained using these techniques have been found to agree with each other to within several percent, the slight difference perhaps being attributable to numerical (round-off) errors.

Note that Eq. (4.2.5) can be re-written as

$$
\sigma_{e l}=\sum_{l=0}^{l_{\max }} \sigma_{e l}^{l}
$$


The calculation evaluates the sum up to $l=l_{\max }$, where the value of $l_{\max }$ depends on the energy of the particle. For the case of particles incident at low kinetic energies, greater numbers of partial waves are required to ensure accuracy. For the case of particles incident at high kinetic energies, such as greater than $10 \mathrm{keV}$, fewer partial waves are required and, by appropriate selection of $l_{\max }$, computation time may be significantly reduced. In practice, we have found that choosing $l_{\max }$ according to

$$
\frac{\sigma_{e l}^{l_{\max }}}{\sum_{l=0}^{l_{\max }} \sigma_{e l}^{l}} \leq 0.001
$$

has given good agreement with values of $\sigma_{e l}$ obtained from other calculations. ${ }^{1}$

The methods employed in the solution of the radial Schrödinger equation and computation the phase shifts have been refined and extensively tested. Results have been generated for a wide range of potentials, including simple potentials for which there exist analytical results. In all cases, agreement between the numerical and analytical forms was excellent. A detailed comparison of the TCS and DSCS for positrons with the results of a similar calculation was made and the agreement was found to be excellent. ${ }^{48,49,50,51,52} \mathrm{We}$ mention again, however, that the particular elastic scattering calculation we have chosen is easily replaced, owing to the extensibility of the underlying program (SPRITE) which performs the transport calculation.

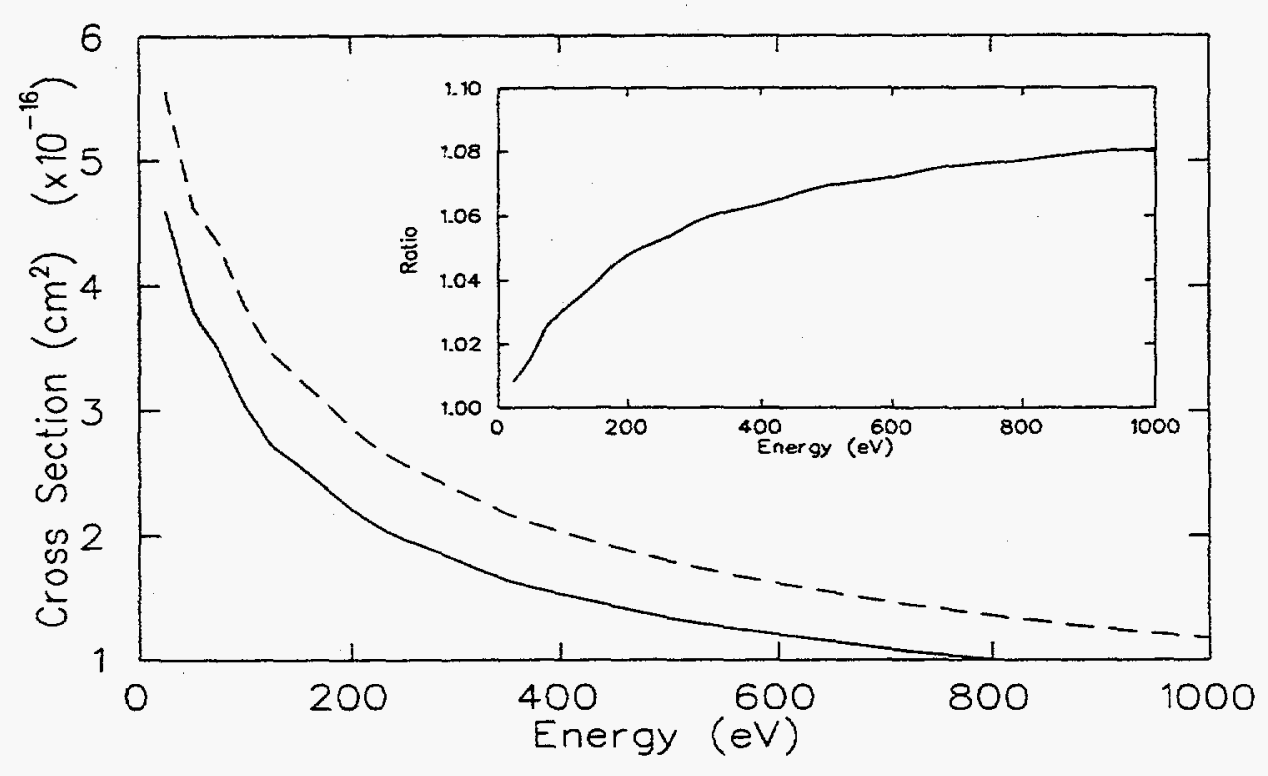

Figure 4.3 The total cross section for the elastic scattering of positrons (solid line) and electrons (dashed line) from Al, as calculated by PSIGEL. The inset is the ratio of the total cross sections calculated by Eq. (4.2.4) and Eq. (4.2.5).

Atomic potentials. The program PATOMIC can be used to generate atomic poten- 
tials, or the user can provide atomic potentials obtained from other sources. In program PATOMIC, the atomic potentials required for the calculation of the DSCS are computed using a modified version of the Dirac-Hartree-Slater-Fock method developed by Liberman, Cromer, and Waber. ${ }^{53,54}$ In this method, the relativistic Dirac equation ${ }^{47}$

$$
\left[\alpha \cdot p+\beta m c^{2}+V(r)\right] \psi_{i}=E_{i} \psi_{i}
$$

is solved self-consistantly for a single multi-electron atom. Here $\alpha$ denotes the $4 \times 4$ Pauli matrices, $p$ is the nomentum operator and $\psi_{i}$ is the Lorentz spinor form of the wavefunction. The potential $V$ includes terms which account for Coulomb interaction of the incident particle (either a positron or an electron) with the atomic nucleus and electron charge distribution. In the case of incident electrons, $V$ contains an additional "exchange" term (using the usual Slater $\rho^{1 / 3}$ form), to account for exchange-correlation effects. Because both $\psi_{i}$ and $V$ are not known initially, Eq. (4.2.8) must be solved self-consistantly. A trial $V$ is used to obtain the orbital wavefunctions (expressed as two-component spinors); these are then used to compute the charge density and, from this, an improved estimate of $V$ is calculated. This process is repeated until convergence is obtained. We have found this program to be quite stable for most cases of interest.

In order to account approximately for the effects of the lattice, the single atom potentials generated by PATOMIC (or obtainied from other sources) can be corrected with a second program, PVCALC. This program renormalizes the potential in one of a variety of ways; for example, charge neutrality can be maintained within the Wigner-Seitz sphere by demanding that the gradient of the potential vanish at $r_{w s}$. The recommended normalization option is to subtract the value of the potential at $r_{w s}$, so that $V\left(r=r_{w s}\right)=0$.

Finally, the program PSIGEL accepts the corrected atomic potentials, performs the partial wave calculation (including the calculation of the spherical Bessel and Neumann functions, as discussed above) and generates the DSCSs and TCSs. By way of example, the atomic potential (computed using PATOMIC) and the renormalized atomic potential (computed using PVCALC) for aluminum $(Z=13)$ are shown in Figures 4.4-4.5. The total cross section, and a differential cross section for a fixed energy, corresponding to this potential and computed using PVCALC and PSIGEL are presented in Figures 4.2-4.3. The use of these programs is discussed in greater detail in files supplied with the source code distribution. 


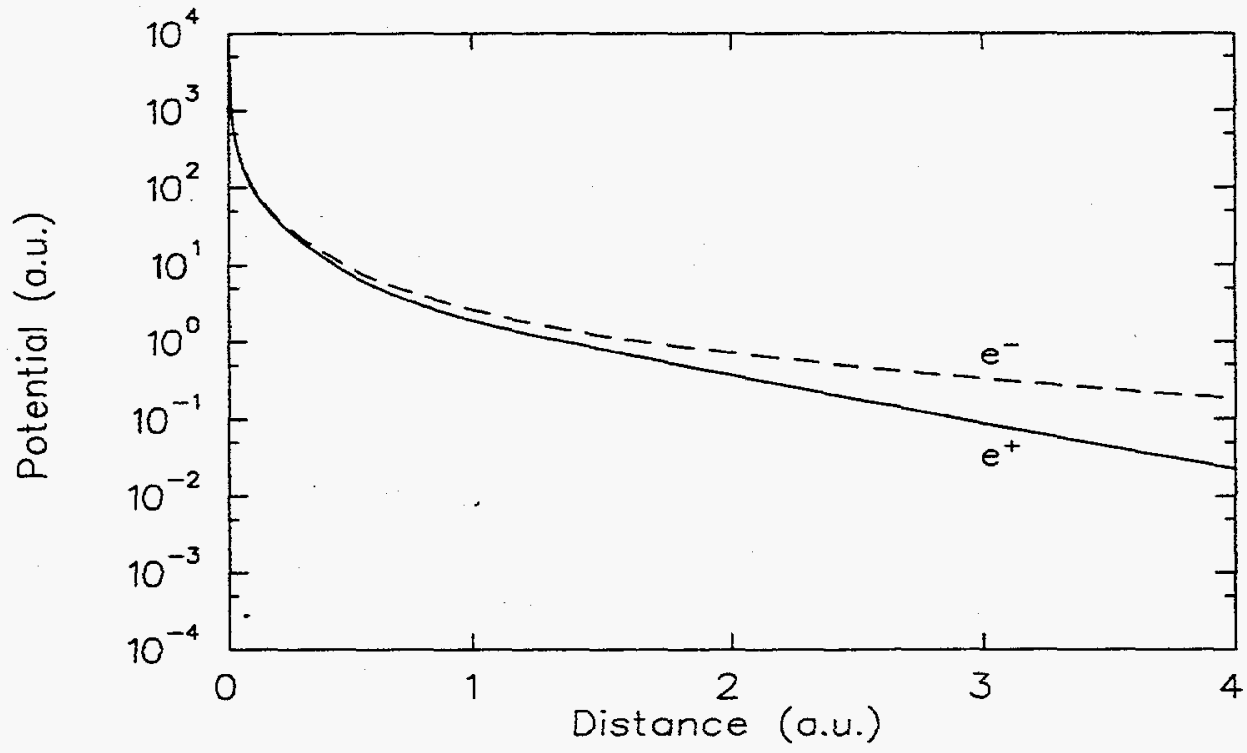

Figure 4.4 The atomic potential for aluminum $(Z=13)$, as calculated by PATOMIC, for positrons (solid line) and electrons (dashed line).

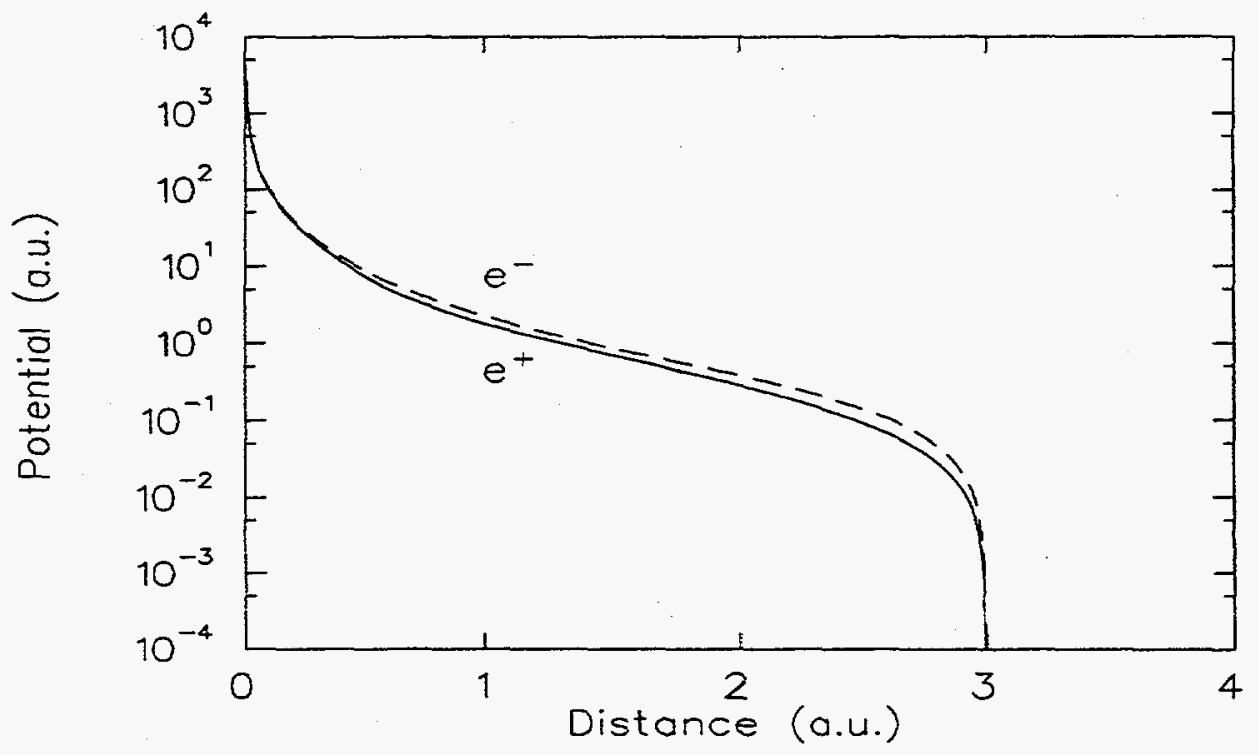

Figure 4.5 The renormalized atomic potential for positrons (solid line) and electrons (dashed line), as calculated by PVCALC. The Wigner-Seitz radius for $\mathrm{Al}$ is $2.995 a_{0}$. 


\subsection{Inelastic Scattering}

\subsubsection{Overview}

Information about inelastic scattering is provided via a user-defined subroutine attached to SPRITE. This subroutine accepts as input the positron or electron energy and the type of solid in which the particle resides, together with information about the user's preferences for scattering mechanisms, and returns information about the scattering cross section (or equivalently, by way of Eq. (2.2.6), mean free path), energy loss per collision, and scattering angle per collision. The mechanisms employed to obtain these three quantities are discussed in detail below. In general, two types of inelastic scattering mechanisms are considered: core-level scattering, such as positron- or electron-scattering with the core electrons of the atoms in the host lattice; and valence-level scattering, such as positron- or electron-interaction with the delocalized electrons of the lattice, plasmons, etc.

\subsubsection{Calculation of Cross Sections}

\subsubsection{K-shell core-level scattering}

The results of Kolbenstvedt are used in the calculation of inelastic scattering due to K-shell ionization. ${ }^{55}$ This mechanism is an important effect when the positron energy is greater than the ionization energy of the $\mathbb{K}$-shell. This total inelastic cross section for relativistic scattering includes contributions from processes at large and small impact parameters. By considering ionization via a virtual photoeffect, the cross section for large impact parameter $b$ is

$$
\sigma(b>a)=\int_{I}^{k_{0}} \sigma_{k} N_{k} d k
$$

where $\sigma_{k}$ is the photoelectric cross section for a photon of energy $k, I$ is the $\mathrm{K}$-shell ionization energy, $N_{k}$ is the field of an electron of velocity $\beta$ passing the atomic nucleus,

$$
d N_{k} \approx(2 \alpha / \pi)\left(d k / \beta^{2} k\right) \log \left(k_{0} / k\right)
$$

and $a$ is the effective $\mathrm{K}$-shell radius,

$$
a=\frac{1}{\alpha Z}
$$

Here $k_{0}$ is the cutoff energy, with $b_{0}$ as the smallest impact parameter, taken to be the $\mathrm{K}$-shell radius:

$$
k_{0}=\left(1.123 \epsilon \beta / b_{0}\right) \exp \left(-\frac{1}{2} \beta^{2}\right)
$$

To obtain the cross section for small impact parameters, $\sigma(b<a)$, the calculation includes both the Moller cross section for incident electrons, as well as the Bhabha cross 
section for incident positrons. In the case of incident electrons, the Moller cross section is calculated from the following:

$$
\sigma(b<a)=2 \int_{w=I /(\epsilon-1)}^{1 / 2} d \sigma_{M}
$$

where the differential scattering element $d \sigma_{M}$ is defined in terms of the ratio $w$ between the energy transfer and the kinetic energy of the incident electron ${ }^{56,57}$

$$
d \sigma_{M}=\frac{2 \pi r_{0}^{2}}{\beta^{2}(\epsilon-1)}\left\{\left(1 / w^{2}\right)+(1-w)^{-1}+(\epsilon-1 / \epsilon)^{2}-\frac{2 \epsilon-1 / \epsilon^{2}}{w(1-w)}\right\} d w
$$

It is worthwhile to note that the factor of 2 in Eq. (4.3.6) derives from the occupancy of the $\mathrm{K}$-shell, and the upper limit in the integral derives from assuming the scattered electron to be the one with the greatest velocity after the collision. In the case of incident positrons, the cross section is computed in a similar manner, but using the Bhabha differential scattering cross section instead, 58,59

$$
\begin{aligned}
d \sigma_{B}= & \frac{\chi}{T}\left\{\frac{1}{e^{2}}-\frac{\gamma^{2}-1}{\gamma^{2} \epsilon}+\frac{1}{2}\left(\frac{\gamma-1}{\gamma}\right)^{2}-\left(\frac{\gamma-1}{\gamma+1}\right) \times\right. \\
& \times\left[\frac{\gamma+2}{\gamma \epsilon}-2 \frac{\gamma^{2}-1}{\gamma^{2}}+\epsilon\left(\frac{\gamma-1}{\gamma}\right)^{2}\right]+ \\
& \left.+\left(\frac{\gamma-1}{\gamma+1}\right)^{2}\left[\frac{1}{2}+\frac{1}{\gamma}+\frac{3}{2 \gamma^{2}}-\left(\frac{\gamma-1}{\gamma}\right)^{2} \epsilon(1-\epsilon)\right]\right\} d \epsilon
\end{aligned}
$$

The total cross section for both large and small impact parameters is thus

$$
\sigma_{k}=\sigma(b>a)+\sigma(b<a)
$$

Values of the electron ionization energy must be specified in the input file by the user; earlier results have used ionization energies which have been taken from the compilation of Williams. ${ }^{60}$

The cross section for positron scattering from the $\mathrm{K}$-shell of aluminum is shown in Figure 4.6.

The current elastic scattering calculation supplied with POS-SPRITE has a maximum calculable energy of $10 \mathrm{keV}$, although nothing in POS-SPRITE precludes the validity of the calculation at higher energies. It is at these high energies in which $\mathrm{K}$-shell scattering is important; thus this formalism will primarily be of use in circumstances in which the user supplies data for elastic scattering at energies higher than these. (Note that at higher energies, however, additional complications such as particle channeling, may arise; the ability to treat these are not currently included in POS-SPRITE.)

Note that it is possible for the user to specify that scattering from the $\mathrm{K}$-shell be described by the formalism due to Gryzinski, rather than than due to Kolbenstvedt. This 


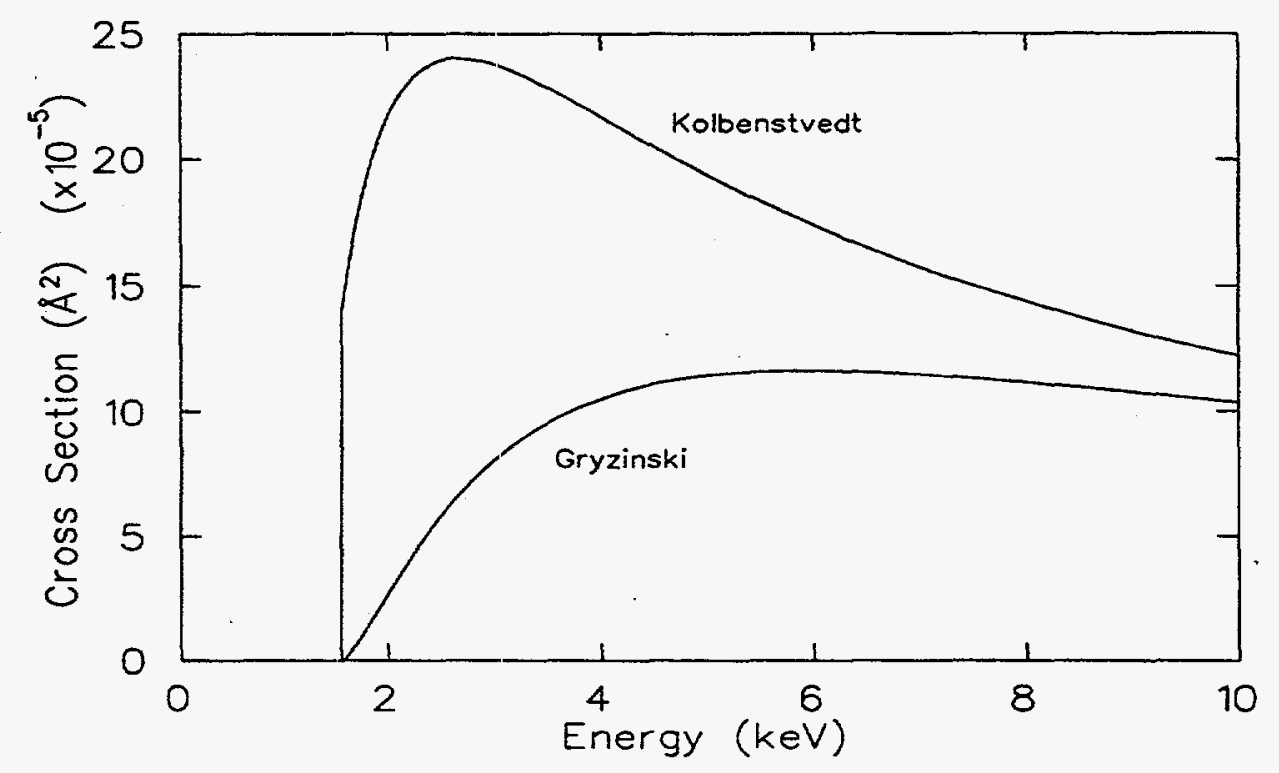

Figure 4.6 The cross sections for positron scattering from the K-shell in aluminum, as calculated by the separate results of Kolbenstvedt and Gryzinski.

option is indicated in Table 4.1. The scattering formalism due to Gryzinski is described in the next section.

\subsubsection{Non-K-shell core-level scattering}

For inelastic scattering from core levels other than the $\mathrm{K}$-shell, and (optionally) to describe $\mathrm{K}$-shell scattering, the cross section Gryzinski excitation function is used. ${ }^{61,62,63}$ This is a description of atomic ionization using a semi-classical two-body description of the process. The total cross section is

$$
\sigma_{i n}=\frac{\pi e^{4} N_{s}}{E E_{B}}\left(\frac{E-E_{B}}{E+E_{B}}\right)^{3 / 2}\left\{1+\frac{2}{3}\left(1-\frac{E_{B}}{2 E}\right) \log \left[2.7+\left(\frac{E}{E_{B}}-1\right)^{1 / 2}\right]\right\}
$$

where $E$ is the kinetic energy of the incident particle, $E_{B}$ is the core-level binding energy, and $N_{s}$ is the number of electrons in a given shell which contribute to the elastic scattering. A separate mean free path for each atomic shell is determined via Eq. (4.3.9), and these are treated as different "mechanisms" in the context of the event selection process, Eq. (2.2.1).

The cross section for positron scattering from aluminum as calculated using Eq. (4.3.9) is shown in Figure 4.6 for $\mathrm{K}$-shell, and in Figure 4.7 for the non-K-shell core levels.

\subsubsection{Valence-level inelastic scattering}




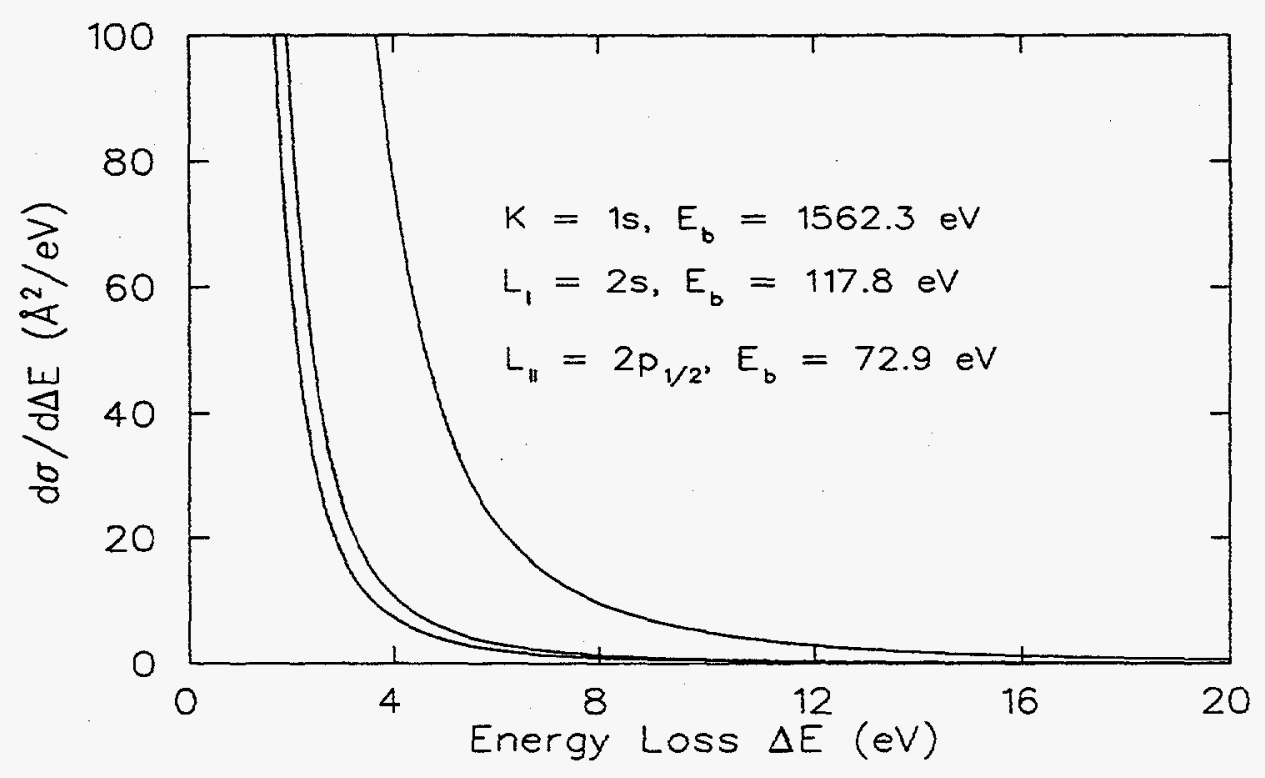

Figure 4.7 The cross section of a positron or electron in aluminum, for each of the non-K-shell core electronic levels, as calculated by the results of Gryzinski.

The scattering of incident positrons and electrons by the valence-level electrons of the host lattice makes an important contribution to the endpoint distribution of the scattered particles. In general, valence-level scattering occurs by two processes, the excitation of collective "plasmon" modes, and the excitation of single-particle "quasiparticle-quasihole" modes. Detailed theoretical formulae for the mean free paths due to these processes exist; these depend on the assumption of a specific form of the dielectric constant $\epsilon(\mathbf{q}, \omega)$ to characterize the electron gas.

The most well-known calculation of the valence mean free path is due to Quinn. ${ }^{64}$ The formalism incorporated into our model is an extension of this due to Penn, which surpasses the original calculation by incorporating exchange and correlation effects. ${ }^{65}$ The mean free path calculated in this way can be parameterized by the following functional form:

$$
\lambda_{\text {Penn }}=\frac{E}{a_{\nu} \log E+b_{\nu}}
$$

where $E$ is the kinetic energy of the incident electron, and $a_{\nu}$ and $b_{\nu}$ are material-dependent parameters which characterize the scattering. Values of the parameters have been reported by Penn [Penn, 1976]. It can be shown that the form of Eq. (4.3.10) is valid for all materials, including transition metals, nobel metals, and rare earths. A problem exists, however, in that the constants $a_{\nu}$ and $b_{\nu}$ can be obtained only when the dielectric function of the material is known, thus making the application of Eq. (4.3.10) to transition, noble, and rare earth metals difficult; these are systems in which the detailed electronic structure is complex and the dielectric function is not easily derived from first-principle calculations. Nevertheless, Eq. (4.3.10) has been compared to more recent calculations in noble metals, 
and found to give agreement to better than $20 \% .{ }^{65}$

We point out that several recent theoretical advances have led to a new description of valence-level scattering, in which information about the optical dielectric function $\epsilon(0, \omega)$ can be used in the calculation of the mean free path. ${ }^{66-70}$ In general this quantity has been measured for a large number of systems, thus this model can be applied to systems other than simple metals. ${ }^{71}$ Unfortunately, experimental confirmation of the effectiveness of this new approach has not been as easily forthcoming. A future extension of POS-SPRITE may be to incorporate this model; at the present time, based on a lack of substantive experimental confirmation, we do not feel that the omission of this description of scattering is a detriment to our calculation.

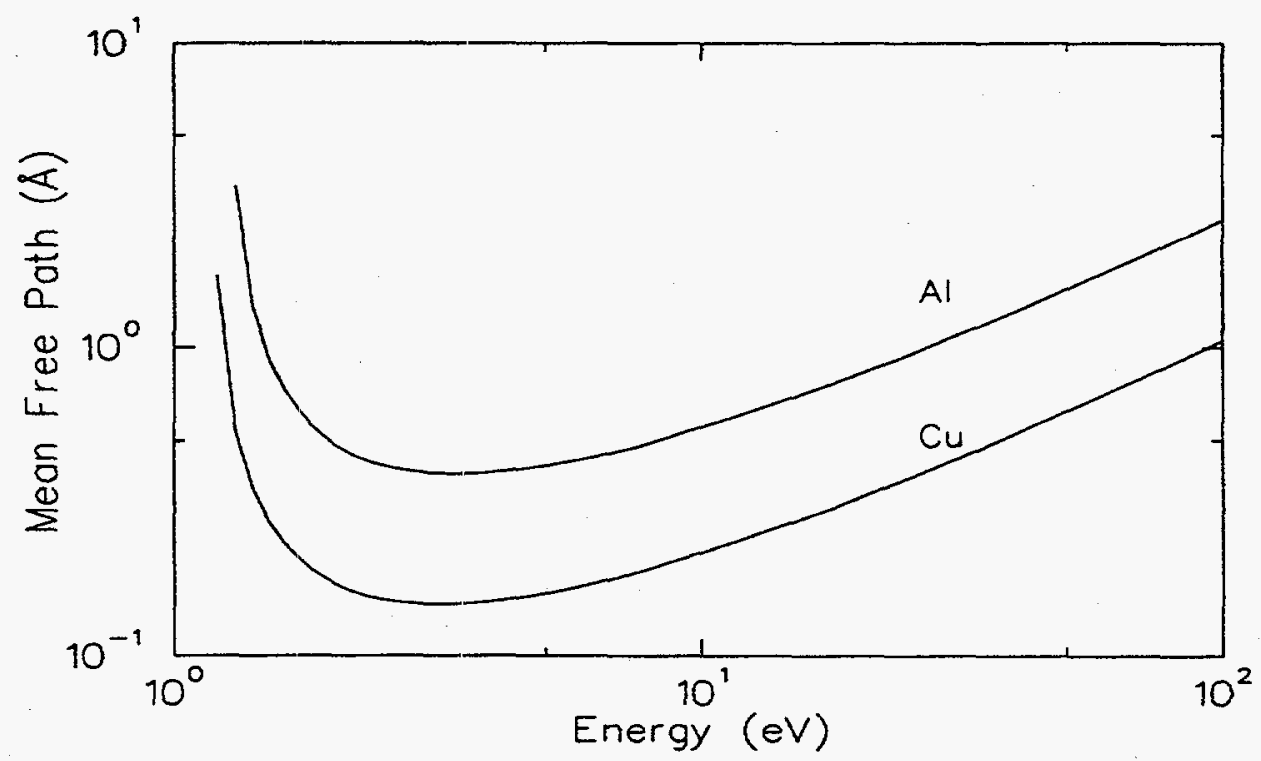

Figure 4.8 The mean free path of a positron and electron in aluminum and copper for valence level scattering, calculated using the results of Penn.

\subsubsection{Calculation of Energy Loss}

If a specific scattering mechanism has been selected by PSPRITE to be one of those discussed in the previous section (i.e., not elastic), the corresponding energy loss is then computed, using results discussed in this section.

\subsubsection{Core-level Energy Loss}

To compute the energy loss due to core-level excitation, the fractional energy transfer for the collision is obtained from 


$$
R=\frac{1}{\sigma_{\mathrm{tot}}} \int_{\epsilon}^{\epsilon \max } \frac{d \sigma^{ \pm}}{d \epsilon^{\prime}} d \epsilon^{\prime}
$$

There are several theoretical approaches to the determination of the differential scattering cross section. Rather than arbitrarily selecting one such description, the calculation includes several; thus the user can explore how the final results are affected by the choice of scattering mechanism, with a view to understanding the relative importance of a given inelastic energy loss mechanism to the overall scattering process.

An approximation to the differential scattering cross section due to Moller scattering (for electrons) and Bhabha scattering (for positrons), as been developed by Rohrlich and Carlson: ${ }^{59}$

$$
\frac{d \sigma^{ \pm}}{d \epsilon}=\frac{2 \pi r_{0}{ }^{2}}{\beta^{2}(\gamma-1)} \frac{1}{\epsilon^{2}} \exp \left(-c^{ \pm} \epsilon\right)
$$

where $\beta$ is the particle velocity relative to the speed of light, $\gamma-1$ is the linetic energy of the particle relative to its rest mass, $r_{0}$ is the classical electron radius and $\epsilon$ is the fractional transfer of energy in the collision. The functions $c^{ \pm}$for positrons $(t)$ and electrons $(-)$ are defined by

$$
c^{+}=\beta^{2}\left[2-(\gamma-1)^{-2}\right] c^{-}=(2 \gamma-1) \gamma^{2}
$$

It is also possible to use the differential cross section due to Gryzinski, ${ }^{61,62,63}$

$$
\begin{aligned}
& \frac{d \sigma^{ \pm}}{d \epsilon}=\frac{\pi e^{4}}{\Delta E^{3}} \frac{E_{b}}{E}\left(\frac{E}{E+E_{b}}\right)^{3 / 2}\left(1-\frac{\Delta E}{E}\right)^{E_{b} /\left(E_{b}+\Delta E\right)} \times \\
& \times \quad\left\{\frac{\Delta E}{E_{b}}\left(1-\frac{E_{b}}{E}\right)+\frac{4}{3} \log \left[2.7+\left(\frac{E-\Delta E}{E_{B}}\right)^{1 / 2}\right]\right\}
\end{aligned}
$$

\subsubsection{Valence-level Energy Loss}

The treatment of valence-level inelastic scattering is slightly more complicated than the core-level case, owing to the two possible modes in which this scattering can occur, namely, the single particle mode and the collective (plasmon) mode. Once PSPRITE has determined that a valence-level inelastic event has occured, the specific mode must be selected prior to the computation of energy loss or scattering angle. This selection is accomplished as follows. First, the mean free path $\lambda_{s p}$ due to single-particle electron scattering is computed using the Penn result discussed above (Eq. (4.3.10)), and the mean free path due to plasmon scattering is computed according to the result of Quinn:64

$$
\lambda_{\text {plas }}=\frac{2 a_{0} E / E_{\text {plas }}}{\log \frac{\sqrt{\left(1+E_{\text {plas }}\right)}-1}{\sqrt{E}-\sqrt{\left(E-E_{\text {plas }}\right)}}}
$$




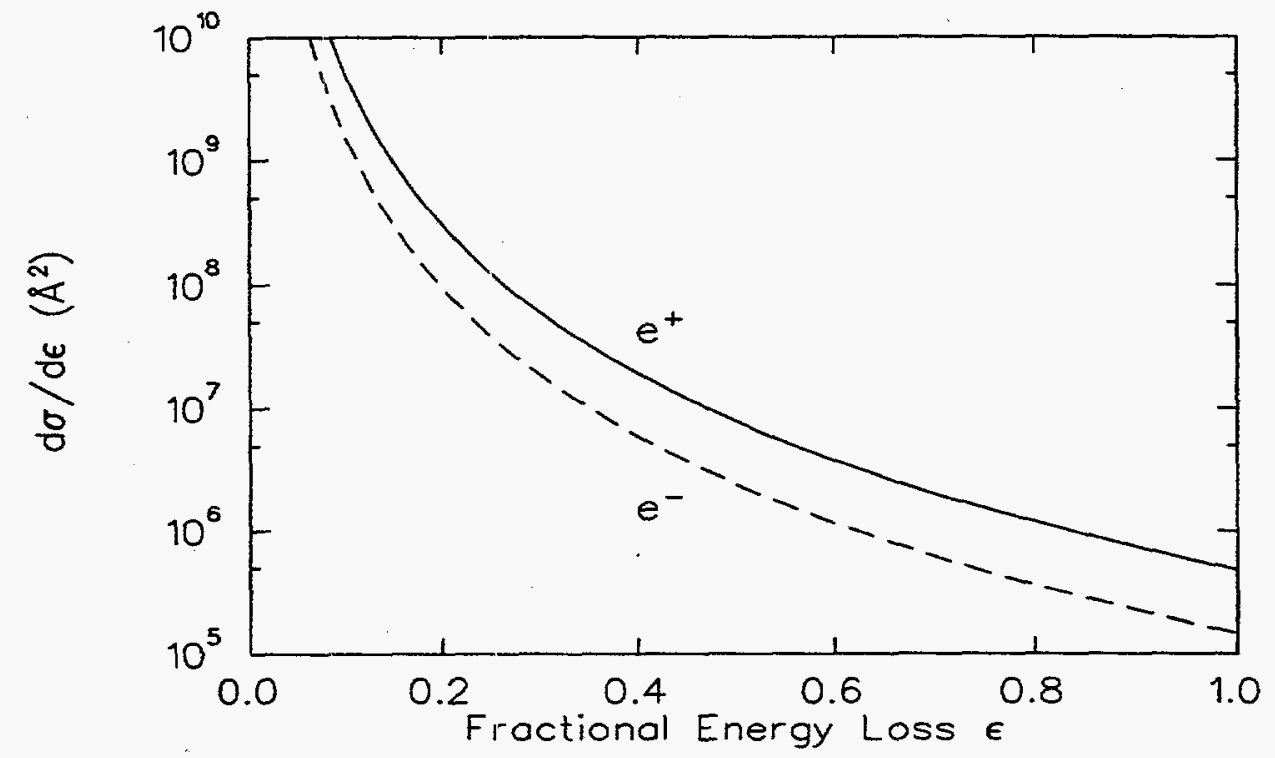

Figure 4.9 The energy-dependent differential scattering cross section, used in the computation of core-level energy loss, calculated using the results of Rohrlich and Carlson for positrons (solid line) and electrons (dashed lines).

where $E_{\text {plas }}$ is the plasmon energy and $a_{0}$ is the Bohr radius. This result was originally developed in the context of electron-plasmon scattering, but recently Oliva has shown that the identical form obtains in the case of positron-plasmon scattering. ${ }^{72,73,74}$

Next, the mode is selected in a manner analogous to that discussed in the context of Eq. (2.2.1). Given a uniformly distributed random number $R$, and the ratio of mean free paths $f=\lambda_{\text {tot }} / \lambda_{\text {plas }}$, the event type is chosen via

$$
\text { event type }= \begin{cases}\text { plasmon, } & 0 \leq R<f \\ \text { single-particle, } & f \leq R<1\end{cases}
$$

Plasmon excitation. If the scattering event is determined to proceed in the collective plasmon mode, then the energy loss is taken to be the plasmon energy of the solid,

$$
\Delta E=\hbar \omega_{p}
$$

Tables of the plasmon energy for a large number of elements are incorporated directly into the PSPRITE code for maximum convenience in use; these values have been taken from the compilation by Isaacsen. ${ }^{75}$ It is possible and simple for the user to alter these values if so desired.

Note that there is a probability that two plasmons will be simultaneously excited in the scattering process, but the probability for this to occur is small $\left(\sim 4 \%\right.$ for $\left.r_{s}=2\right)$ and thus this process is excluded from the calculation. ${ }^{76}$ There is also a probability that surface 
plasmons will be excited $\left(\omega_{s p}=\omega_{p} / \sqrt{2}\right)$, but due to the supression of bulk plasmons near the surface this process is similarly excluded.

Single particle excitation. If the scattering event is determined to proceed in the singleparticle excitation mode, the energy loss is computed in a manner analogous to Eq. (4.3.11) but using a description of the valence electrons in the random phase approximation (RPA). The differential scattering cross section is written ${ }^{69}$

$$
\frac{d \sigma(\omega)}{d \omega}=\frac{2 e^{2}}{\pi \hbar^{2} v^{2}} \int_{k^{-}}^{k^{+}} \frac{d k}{k} \Im\left[-\frac{1}{\epsilon(k, \omega)}\right]
$$

where $\omega$ is the energy of the particle, $\hbar \omega$ is the energy transfer to the material, $v$ is the velocity of the particle, and the allowable limits of momentum transfer $k^{ \pm}$are given by

$$
k^{ \pm}=\sqrt{\frac{2 m E}{\hbar^{2}}}(\sqrt{E} \pm \sqrt{E-\hbar \omega})
$$

In this calculation, the dielectric response function (which contains information about the correlation effects of a many-body system) $\epsilon(k, \omega)$ is required. A large number of such functions have been determined theoretically, the most well-known of which is that due to Lindhard;77 this is also the simplest function which correctly describes the collective oscillations (plasmons) of a free-electron gas. Several authors have shown, however, that the contributions to energy-loss models (such as ours) which result from different choices of dielectric function differ negligibly from the Lindhard model, at least in the case of metals and for electron energies above the Fermi level. ${ }^{69}$ Therefore we adopt the Lindhard function, with real and imaginary parts given by

$$
\begin{gathered}
\epsilon_{r}(k, \omega)=1+\frac{2 m e^{2} p_{0}}{\pi k^{2}}\left\{1-\frac{p_{0}}{2 k}\left[1-\left(\frac{\omega m}{p_{0} k}-\frac{k}{2 p_{0}}\right)^{2}\right] \log \left|\frac{1+\frac{\omega m}{p_{0} k}-\frac{k}{2 p_{0}}}{1-\frac{\omega m}{p_{0} k}+\frac{k}{2 p_{0}}}\right|\right. \\
+\frac{p_{0}}{2 k}\left[1-\left(\frac{\omega m}{p_{0} k}+\frac{k}{2 p_{0}}\right)^{2}\right] \log \mid \frac{1+\frac{\omega m}{p_{0} k}+\frac{k}{2 p_{0}}}{\left.1-\frac{\omega m}{p_{0} k}-\frac{k}{2 p_{0}} \mid\right\}} \\
\epsilon_{i}(k, \omega)=\left\{\begin{array}{c}
\frac{m e^{2} p_{0}^{2}}{k^{3}}\left[1-\left(\frac{\omega m}{p_{0} k}-\frac{k}{2 p_{0}}\right)^{2}\right], \\
\frac{k}{p_{0}}+\frac{1}{2}\left(\frac{k}{p_{0}}\right)^{2} \geq \frac{\omega m}{p_{0}^{2}} \geq\left|\frac{k}{p_{0}}-\frac{1}{2}\left(\frac{k}{p_{0}}\right)^{2}\right| \\
\frac{k}{k^{3}}, \quad 0 \leq \frac{\omega m}{p_{0}^{2}} \leq \frac{k}{p_{0}}-\frac{1}{2}\left(\frac{k}{p_{0}}\right)^{2}
\end{array}\right.
\end{gathered}
$$

Here $\omega_{0}$ is the plasmon frequency, and $p_{0}$ is the Fermi momentum, $\omega=2 p k x-k^{2}$. 
The energy-dependent probability of interaction, calculated using Eq. (4.3.20) and Eq. (4.3.21) - Eq. (4.3.22) in Eq. (4.3.18), appears in Figure 4.10. The probability densities for several values of $r_{s}$ are shown, where $r_{s}$ is the free-electron radius and is defined by the density $n$ of the free-electron gas,

$$
\frac{4}{3} r_{s}^{3}=\frac{1}{n a_{0}}
$$

where $a_{0}=0.529 / \mathrm{AA}$. The poles result from the collective plasmon excitations of the electron gas, discussed in greater detail below. We note that the energy corresponding to the plasmon excitations is proportionall to $r_{s}^{-3 / 2}$, as expected from elementary considerations (see, for example, Reference 78),

$$
E_{p}=[47.1 \mathrm{eV}] r_{s}^{-3 / 2}
$$

Note that the value of $r_{s}$ can be related to the number of electrons $N$ in the valence level by

$$
r_{s}=1.389\left[\frac{A(\mathrm{~g})}{N \rho(\mathrm{g} / \mathrm{cc})}\right]^{1 / 3}
$$

where $A$ is the atomic weight in $\mathrm{g}$ and $\rho$ is the density in $\mathrm{g} / \mathrm{cc}$; this provides a useful method of obtaining values of calculating the effective valency of transition and nobel metals.

To achieve maximum operational speed, information relevant to the valence-level energy loss calculation is generated by a separate program, POSRPA, prior to performing the PSPRITE calculation. This program prepares a file containing the energy-dependent differential cross sections; PSPRITE reads this file just prior to beginning the transport calculation.

\subsubsection{Calculation of Scattering Angle}

Having completed our elaboration of the calculation of mean free path and energy loss due to inelastic scattering, we conclude by addressing the computation of scattering angle.

\subsubsection{Core-level Scattering Angle}

We must account for the inelastic scattering of high energy electrons or positrons with the atomic cores of the host lattice. For a particle suffering this type of inelastic collision, such that its energy $E$ is demoted to energy $E-\Delta E$, the resulting scattering angle $\theta$ has been computed by Gryzinski, using the so-called binary collision approximation. ${ }^{61,62,63}$ The result is

$$
\sin (\theta)=\left(\frac{\Delta E}{E}\right)^{1 / 2}
$$

This result is strictly true in the limit that the energy transfer is a small fraction of the particle energy. During the development of PSPRITE, higher-order terms in the derivation 


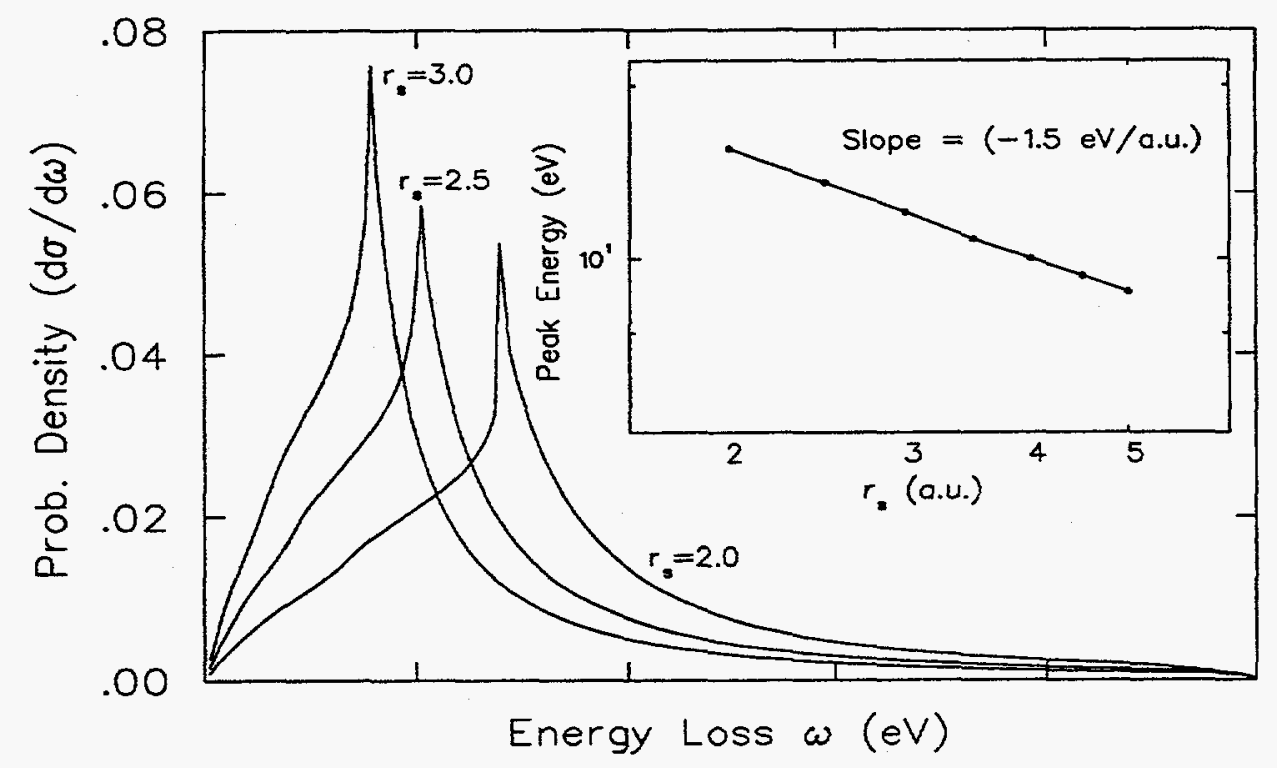

Figure 4.10 The differential cross section for valence-level positron scattering, calculated using a dielectric function obtained in the random phase approximation (RPA). The inset shows the $r_{s}^{-3 / 2}$ dependence of the plasmon excitation energy.

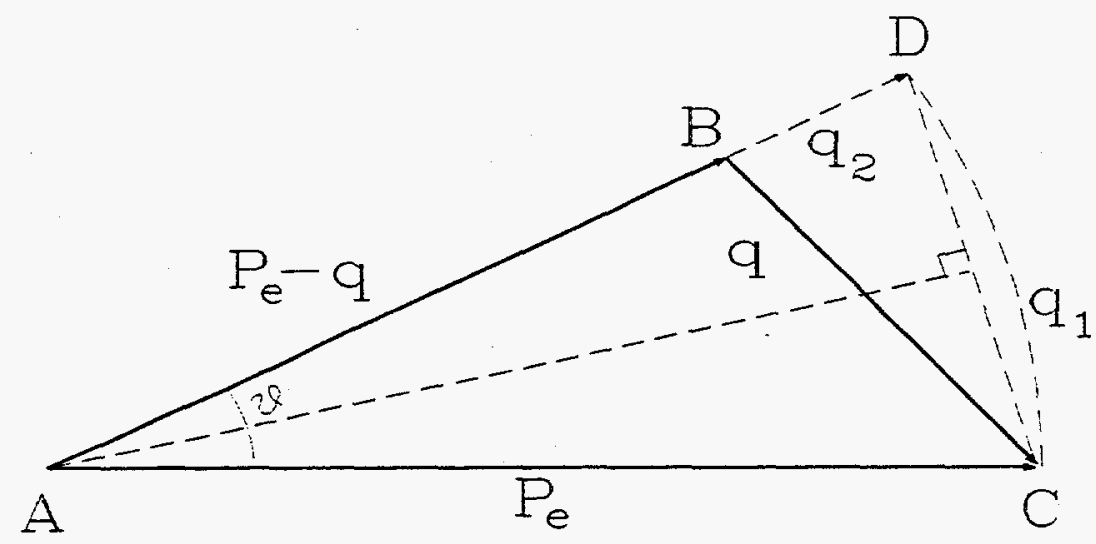

Figure 4.11 A vectorial representation of scattering, in which a particle with momentum $P$ transfers momentum $q$ and energy $\omega$ to the scattering system.

of Eq. (4.3.24) were retained, so that a more accurate expression could be derived, but the inclusion of these did not result in a significant effect upon the major results of the calculation (such as the stopping profile and backscattered fraction). 


\subsubsection{Valence-level Scattering Angle}

As in the case of energy loss at the valence level, we must deal separately with singleparticle "quasiparticle-quasihole" excitations and collective "plasmon" excitations. The scattering angle is computed by randomly sampling according to the differential scattering cross section, in a manner analogous to that discussed in reference to Eq. (2.3.4),

$$
R=\frac{2 \pi}{\sigma_{\text {tot }}} \int_{0}^{\epsilon_{\max }} \frac{d \sigma^{ \pm}}{d \theta} d(\cos \theta)
$$

To apply this, we need the differential scattering cross section for the two types of scattering. The differential scattering cross section for both modes can be easily calculated according to the many-body theory of interactions with the electron gas. The derivation is elegant and it clearly demonstrates the interrelationship of the single-particle and collective modes. We provide a brief outline of the derivation here, and we refer the interested reader to several sources where additional information can be obtained. ${ }^{77,79,80}$

The starting point for the derivation of the differential scattering cross section for electron and positron scattering with the electron gas is Fermi's Golden Rule,

$$
P(\mathbf{q}, \omega)=2 \pi\left(\frac{4 \pi e^{2}}{q^{2}}\right) S(\mathbf{q}, \omega)
$$

where $P(q, \omega)$ is the transition probability that the electron or positron will transfer momentum $\mathbf{q}$ and energy $\omega$ to the electron gas, and $S(\mathbf{q}, \omega)$ is the dynamic form factor, which describes all properties of the electron gas which are relevant to the scattering. We desire information about solid scattering angle $\Omega$, rather than momentum transfer $q$, so that the information contained in Figure 4.11, namely,

$$
q_{1}=P_{e}\left[\theta^{2}+\left(\omega / m V_{e}^{2}\right)^{2}\right]^{1 / 2}
$$

can be used to write Eq. (4.3.26) as

$$
P(\Omega, \omega)=\frac{4 e^{2}}{a_{0} P_{e}^{3}} \frac{1}{\left[\theta^{2}+\left(\omega / m V_{e}^{2}\right)^{2}\right]^{2}} S\left(q_{1}, \omega\right)
$$

To proceed further, we need to evaluate the dynamic form factor. The dynamic form factor can be written in terms of the dieletric response function, which also characterizes the electron gas,

$$
\begin{aligned}
S(\mathbf{q}, \omega) & =\frac{q^{2}}{4 \pi^{2} e^{2}} \times \operatorname{Im}\left(\frac{1}{\epsilon(\mathbf{q}, \omega)}\right) \\
& =\frac{q^{2}}{4 \pi^{2} e^{2}} \times \operatorname{Im}\left(\frac{1}{\epsilon_{1}+i \epsilon_{2}}\right) \\
& =\frac{q^{2}}{4 \pi^{2} e^{2}} \times \frac{\epsilon_{2}}{\epsilon_{1}^{2}+\epsilon_{2}^{2}}
\end{aligned}
$$

In our calculation, we employ the Lindhard or random phase approximation (RPA) dielectric function. In the RPA, the imaginary part of the dielectric constant $\epsilon_{2}$ has the property 
that $\epsilon_{2} \rightarrow 0$ as $\omega \rightarrow 0$, so that $S$ has maxima for $\epsilon_{1}=0$. These maxima correspond to collective "plasmon" oscillations of the electron gas; the frequency of these oscillations in the small $q$ limit is given by

$$
\omega=\omega_{p}\left(1+\frac{3}{10} \frac{q^{2} v_{F}^{2}}{\omega_{p}^{2}}+\cdots\right)
$$

In the "plasmon" regime, the $q^{2}$ term in Eq. (4.3.30) provides a small correction to the plasmon frequency. As $q$ becomes larger (or correspondingly, the scattering angle), the plasmon energy has a larger $q$-dependence and the plasmons themselves become damped. In the approximate neighborhood of a critical momentum $q_{c}$,

$$
q_{c}=\frac{\omega_{p}}{v_{F}}
$$

or critical angle,

$$
\theta_{c}=\frac{1}{4} \frac{\omega_{p}}{v_{F} P_{e}}
$$

the probability of collective oscillations falls off and single-particle excitations become dominant. The excitation spectrum of the electron gas is depicted in Figure 4.12.

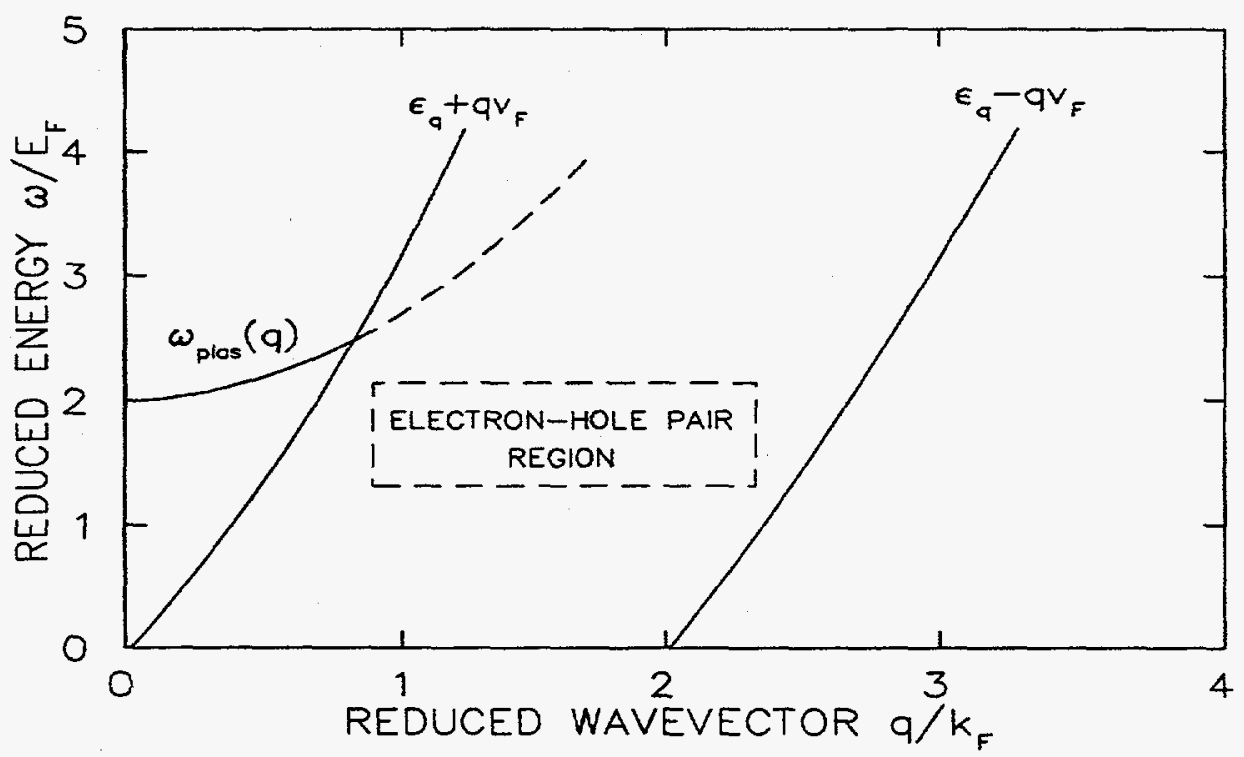

Figure 4.12 The excitation spectrum of the free electron gas, in which the single-particle and plasmon modes can be seen.

We now evaluate $S(q, \omega)$ explicitly. In the limit of small momentum transfers $(\mathbf{q} \rightarrow 0)$, it can be shown that $S(q, \omega)$ satisfies the f-sum rule, 


$$
\int_{0}^{\infty} \omega d \omega S(q, \omega)=\frac{N q^{2}}{2 m}
$$

where $\delta\left(\omega-\omega_{1}\right)$ is the energy-conserving delta function. It is easy to see from Eq. (4.3.33) that

$$
S\left(q_{1}, \omega\right)=\frac{N q_{1}^{2}}{2 m \omega_{1}} \delta\left(\omega-\omega_{1}\right)
$$

Using Eq. (4.3.34) in Eq. (4.3.28), we derive the following:

$$
P(\Omega, \omega)=\frac{\omega_{p}^{2}}{2 \pi a_{0} P_{e}}\left[\frac{1}{\theta^{2}+\left(\omega_{1} / m V_{e}^{2}\right)^{2}}\right] \frac{1}{\omega_{1}} \delta\left(\omega-\omega_{1}\right)
$$

In the single-particle excitation region, $\theta>\theta_{c}, q \approx P_{e} \theta$ and Eq. (4.3.35) becomes

$$
P(\Omega, \omega)=\frac{4 e^{2}}{a_{0} P_{e}} \frac{1}{\theta^{4}} S\left(P_{e} \theta, \omega\right)
$$

Finally, we can obtain what we have intended to derive, the differential scattering cross sections. The total transition probability $P(\Omega)$ is obtained from $P(\Omega, \omega)$ via a coordinate transformation

$$
P(\Omega)=\int_{0}^{\omega} P(\Omega, \omega) d \omega
$$

Using Eq. (4.3.35) and Eq. (4.3.36) in Eq. (4.3.37), and adopting the more usual notation for $P(\omega)$, we find, for both the plasmon and single-particle regimes,

$$
\frac{d \sigma}{d \Omega}= \begin{cases}\frac{\omega_{p}}{2 x a_{0} P_{e} \overline{\theta^{2}}+\left(\omega_{p} / m V e^{2}\right)^{2}} & \theta \leq \theta_{i} \\ S\left(P_{e} \theta\right) & \theta \geq \theta_{i}\end{cases}
$$

It is convenient to consider the angle $\theta_{i}$ at which the differential scattering cross sections for the two processes intersect, which is found to be

$$
\theta_{i}=\left(2 \theta_{E}\right)^{1 / 2}
$$

where $\theta_{E}$ is related to the Fermi energy $E_{0}$ via

$$
\theta_{E}=\omega_{p} / E_{0}
$$

Using these, Eq. (4.3.38) can be written as

$$
\frac{d \sigma}{d \Omega}= \begin{cases}\frac{1}{2 \pi n a_{0}} \frac{\theta_{E}}{\theta^{2}+\theta_{E}^{2}} & \theta \leq \theta_{i} \\ \frac{\theta_{E}^{2}}{\pi n a_{0} \theta^{4}} & \theta \geq \theta_{i}\end{cases}
$$

Plasmon excitation. The integration of Eq. (4.3.41) in Eq. (2.3.4) is straightforward and one obtains, after some rearrangement, ${ }^{33}$ 


$$
\theta_{p l a s}=\theta_{E}\left\{\exp \left[\left(\log \left(\frac{2+\theta_{E}}{\theta_{E}}+1\right) R\right]-1\right\}^{1 / 2}\right.
$$

Single particle excitation. In the case of a single-particle excitations, the scattering angle is found to $\mathrm{be}^{33}$

$$
\theta_{s p}=\left\{\frac{2 \theta_{E}}{1-R}\left[\log \left(\frac{2+\theta_{E}}{\theta_{E}}\right)+1\right]^{-1}+1\right\}^{1 / 2}
$$

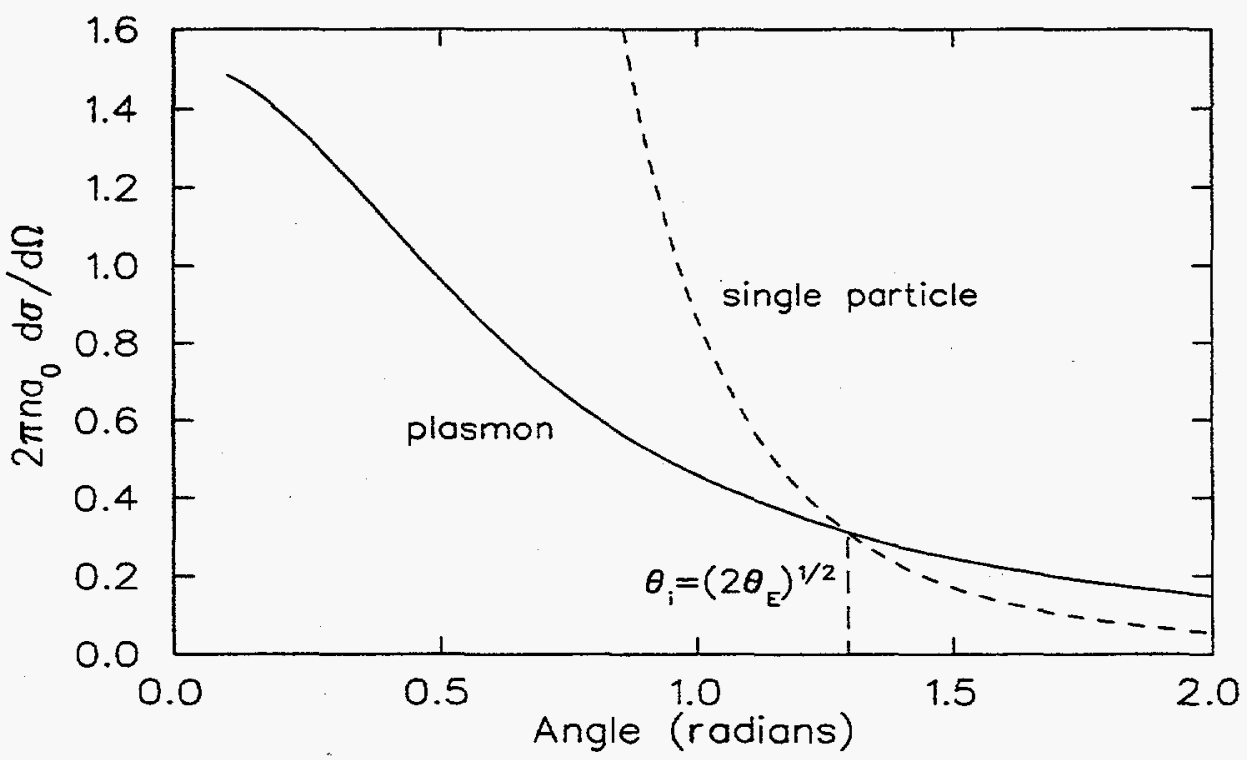

Figure 4.13 The differential cross section for positron-plasmon scattering at two different energies, showing that it is strongly peaked in the forward direction. The inset shows the intersection of the differential cross sections for the plasmon and single-particle excitation processes. 


\subsection{POS-SPRITE Simulation Control File}

The information necessary for PSPRITE is appended to the end of a SPRITE-type simulation control file. An example of this file for the case of an aluminum sample is provided in Figure 4.14. The additional entries required by PSPRITE are well-commented and thus should be self-explanatory. For maximum clarity, however, we discuss the purpose of each required record herewith:

Line 34 This line denotes whether the calculation is to be performed for positrons or electrons.

Line 35 Denotes the number of layers in the system. A complete description of the solid for each layer must follow.

Line 37 The name of the material. The calculation does not use this information; it is provided for the convenience of the user.

Line 13 Atomic number of the material. This is used as an index to obtain information about the element which has been preprogrammed into the SPRITE code.

Line 39 The name of the file which contains the RPA-calculated electron gas scattering factors.

Line 41 Logical flag which specifies whether the valence-level mean free path is to be computed using the calculation by Penn (.TRUE.) or Gryzinski (.FALSE.).

Line 42 Logical flag which specifies whether the core-level energy loss is to be computed using the calculation by Rohrlich and Carlson (.TRUE.) or by Gryzinski (.FALSE.).

Line 43 Logical flag which specifies whether the core-level scattering cross section is to be computed according to the calculation by Kolbenstvedt (.TRUE.) or by Gryzinski (.FALSE.).

Lines 44-45 The ratio of the threshold energy to the binding energy for the core and valence levels.

Lines 46-56 For each core level (and also for the valence level, at the end of the list), the occupation number of the level and the binding energy of the electrons in that level. The name which describes the level (e.g., 1-s, 2-s, etc.) is provided for the convenience of the user and is not used in the calculation. 


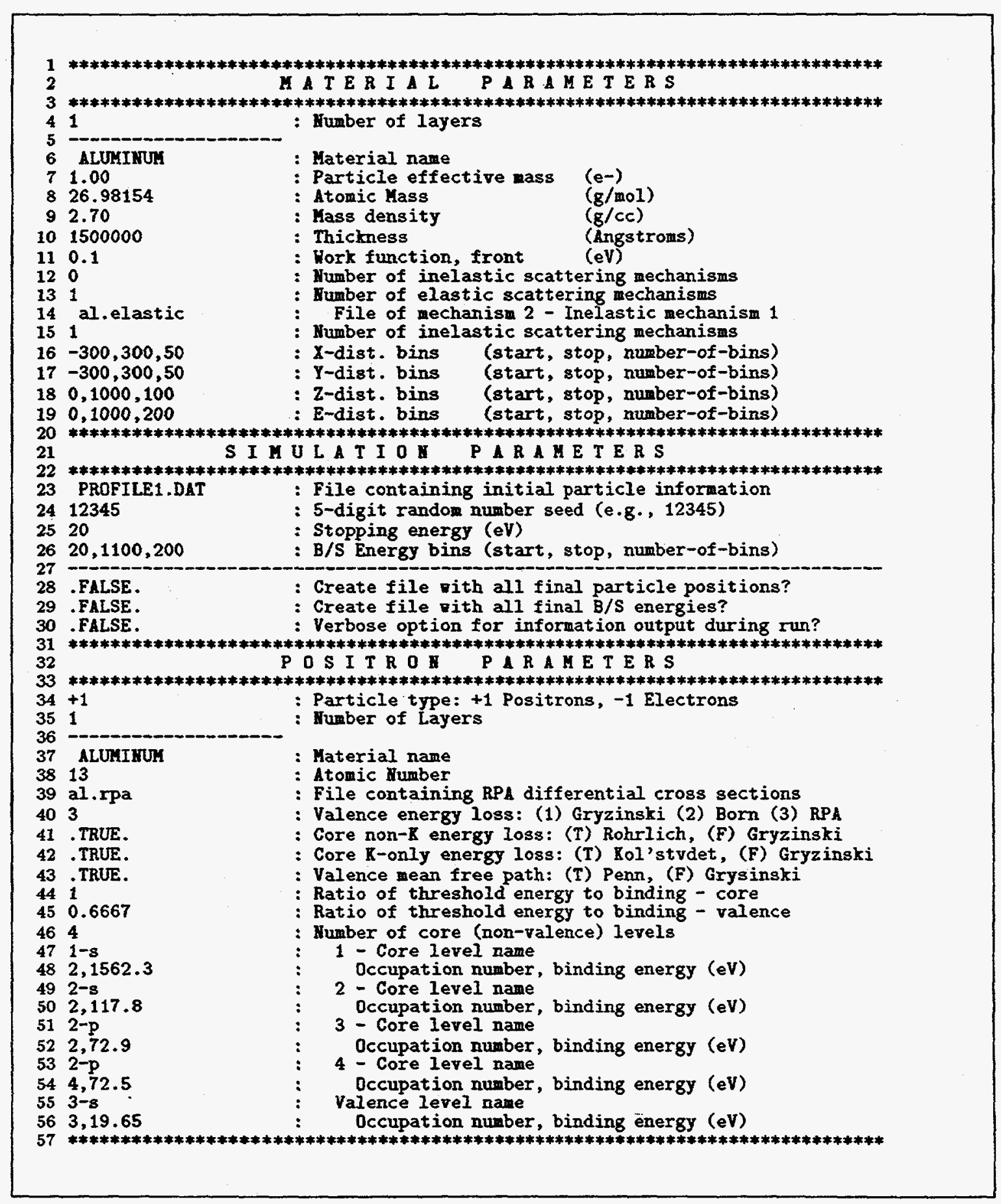

Figure 4.14 An example of the simulation control file required by PSPRITE. This is an ordinary SPRITE control file, with information relevant to positron-solid scattering appended to the end. This file is set up to model a single layer of aluminum. 


\section{Appendix A. The Structure of SPRITE}

This Appendix contains information about the detailed Fortran implementation of the SPRITE program. This is expected to be of assistance to users intending to modify the program. As discussed earlier, SPRITE is designed to be user-friendly and easy-to-modify, even at the cost of execution speed. This Appendix should provide all the information required for the user to tailor the internal operation of SPRITE to meet non-standard requirements.

A keen understanding of several pieces of information about SPRITE is essential to the user who intends to modify SPRIICE. This includes information about the overall program structure (i.e., the main program and the key subroutines - i.e., the building blocks which compose SPRITE - as well as the important global variables. These two topics are discussed below.

\section{Appendix A.1 The Program Structure}

The SPRITE program is currently packaged in two files: a main file (SPRITE3.FOR), which contains the program code and subroutines; and an "include" file (INC3.FOR), which contains the global variable declarations and common block definitions. During the initial design of SPRITE, it was felt that incorporating the global variables into a single include file would facilitate user access to the important global variables and hence simplify program modification, although one obvious drawback to this is that all global variables are then accessible to all subroutines, regardless of whether a given subroutine requires a given set of variables or not. Given that the important global variables follow a rigorous naming convention, this potential drawback is under no circumstances expected to be problematic.

It is assumed at this point that the user has a general understanding of the basic transport calculation as elaborated in Section 2, a block diagram of which appears in Figure 2.1. The structure of the SPRITE program closely parallels this, and we refer the reader to Figure A.1, which contains the main control module of SPRITE. Each of the control steps depicted in Figure A.1 is bound to a single subroutine, as can be seen in Figure D.2. Due to the almost one-to-one correspondance between a functional task outlined in the block diagram, and a subroutine which performs that task, a detailed description of the function of each subroutine is not warranted. A complete hierarchical listing of all routines included in SPRITE is provided in Figure D.1.

\section{Appendix A.2 Global Variables}

The important global variables in SPRITE are all defined and contained in the include file, INC3.FOR. Further, these variables all follow a rigorous naming convention; this reduces the chances for mishap, such as accidental redefinition of a existing variable, or the improper use of an existing variable (such as assigning a floating point value to an integer variable). 


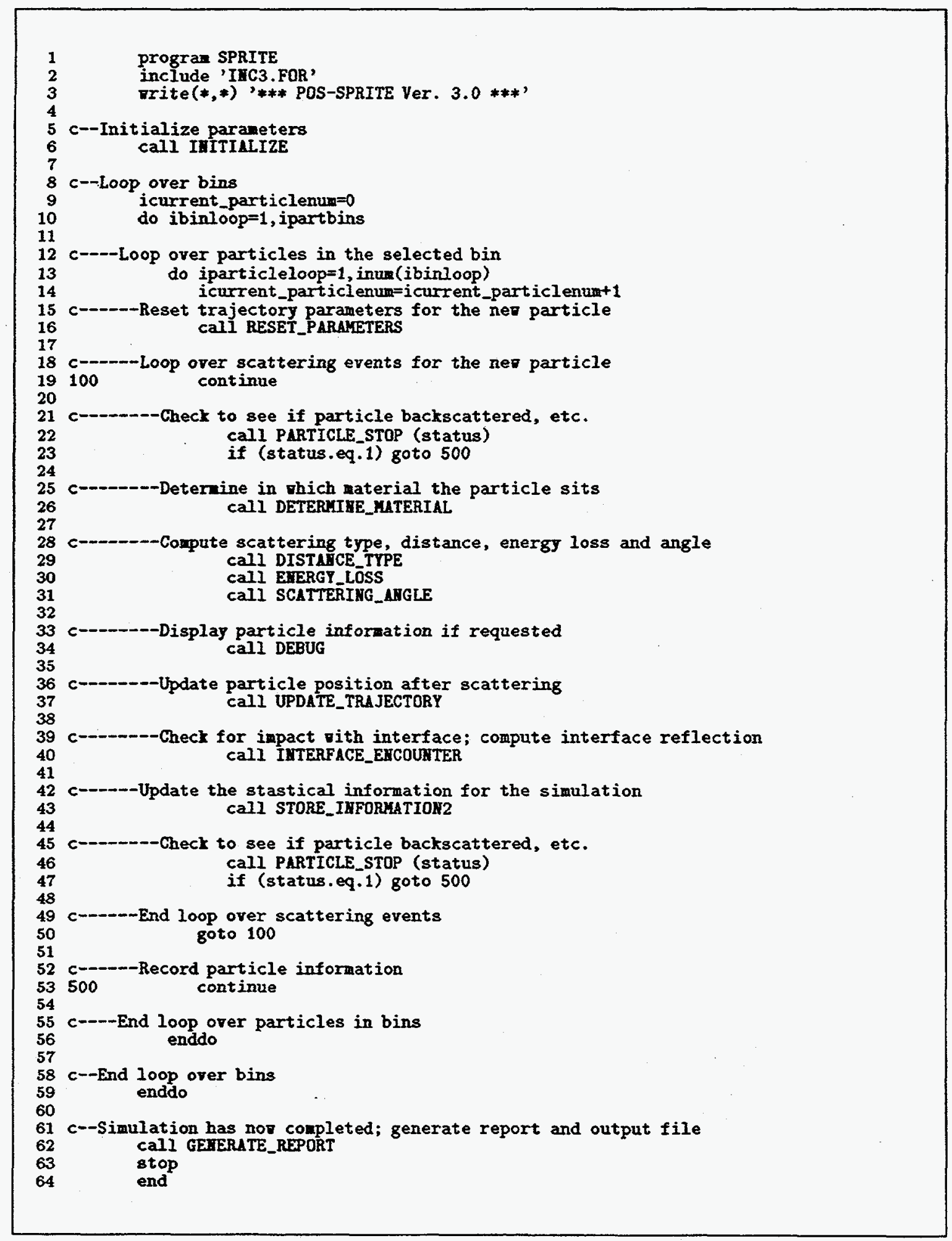

Figure A.1 The main program module of SPRITE, demonstrating the simplicity of the program. 
The first obvious aspect of the laaming convention is that the names of integer variables and logical variables begin with the letters ' $I$ ' and ' $L$ ', respectively; and that the names of character variables which store file names begin with the letter ' $F$ '. The second obvious aspect is that the important global variables possess prefixes (such as TRA JECTORY_ or DEBUG_ or BIN_ ) which indicate the context in which they are commonly used. A complete list of the important global variables, along with a brief description of the physical quantities they represent, is provided in Table D.2.

It is strongly recommended that the user who wishes to modify or extend SPRITE refrain from the casual incorporation of new global variables into the INC3.FOR include file. The construction of a new include file by the user - and the restriction of this file to the exact program modules in which new variables are required - is expected to be easier, and such a procedure will help make clear where the user-modifications are located (i.e., where the original SPRITE stops and where the new code starts). 


\section{Appendix B. Incorporating a User-Defined Subroutine}

\section{Appendix B.1 Overview}

It is anticipated that a common modification desired by a user may be the incorporation of a user-defined subroutine to provide a run-time computation of scattering information, in a manner discussed in Section 2. Although information about the scattering mechanisms can be readily obtained from two types of external data files, the incorporation of a user-defined subroutine gives the user an unlimited degree of flexibility in providing a description of how a particle is to be scattered by the solid. Examples include a time-dependent scattering mechanism, or scattering which depends on the precise particle position within the host lattice. The incorporation of such a subroutine is simple. This is discussed in great detail herewith.

It is recommended that the user decompose the task of a user-defined scattering mechanism into four basic subtasks: 1) initialization prior to program execution, 2) calculation of mean free path, 3) calculation of energy loss, and 4) calculation of scattering angle. We address each of these in turn.

User-initialization. SPRITE facilitates the execution of a user-defined subroutine immediately prior to performing the transport calculation. This provides the user with the ability to perform important tasks before the calculation commences. Such tasks might include, for example, the construction or reading of external data files, or possibly the acquisition of data from the user via the keyboard (standard input device).

As shown in Figure A.1, one of the first subroutines invoked by SPRITE is the subroutine INITIALIZE, which in turn may invoke an initialization routine supplied by the user. An example of such an initialization routine, called USER_INITIALIZE, is actually coded into INITIALIZE, but this routine is currently commented out so that it does not compile. The new subroutine USER_INITIALIZE, if constructed by the user, may contain common blocks or other variable declarations (or better still, an INCLUDE statement), for any new variables which will be part of the user-extended code.

Calculation of mean free path. The mean free path of the particle is first required in subroutine DISTANCE_TYPE. The function of this subroutine is to control the evaluation of the cross sections for each of the mechanisms, and then to use these to select the type of scattering event (according to the results discussed in Section 2). This subroutine invokes subroutine USER_GETSIGS, and it is this subroutine which the user is expected to modify. Although additional subroutines may be created by the user and appended to SPRITE, it is expected that the modification of USER_GETSIGS will involve two steps: first, usercalculated mean free paths will be entered into the array of scattering cross sections (CS) in USER_GETSIGS; second, the variable IUSERSIGS, which indicates the number of userdefined cross sections, will be updated accordingly. For example, if the user has only one cross section to add, then only CS (1) needs to be assigned, followed by the statement IUSERSIGS $=1$. Note that, because of the INCLUDE statement, all of the SPRITE global variables are available in USER_GETSIGS. Thus the user can access these, if desired. 
Calculation of energy loss and scattering angle. The subroutines to compute the userdefined energy loss and scattering angles (USER_ENERGYLOSS and USER_SCATANGLE) function differently than USER_GETSIGS. The subroutine USER_GETSIGS will be invoked prior to each scattering event; this subroutine nust return information about all possible types of userdefined scattering mechanisms. The subroutines USER_ENERGYLOSS and USER_SCATANGLE are invoked only when the SPRITE subroutine DISTANCE_TYPE has determined that a userdefined mechanism has occured. Thus these subroutines need to return information about one scattering mechanism, namely, that which has been determined to occur.

First, these routines need to determine which of the user-defined mechanisms has been found to occur, if there are more than one. This is most easily shown by example: Assume that the user has implemented two new scattering mechanisms, so that USER_GETSIGS has updated the first and second elements of array CS, and has made the assignment IUSERSIGS $=2$. Information about which user-defined scattering mechanism has been found to occur is accessed through the global variables ICURRENT_SCATTYPE, IELSIGS, and INSIGS, and is equal to

$$
\text { ITYPE = ICURRENT_SCATTYPE - IELSIGS - INSIGS }
$$

In this example, ITYPE will either have the value 1 or 2 , depending on which of the userdefined mechanisms DISTANCE_TYFE has calculated should occur.

Second, depending on the chosen type of scattering mechanism, the energy lost per collision needs to be assigned to variable USER_DE in USER_ENERGYLOSS; similarly the cosine of the scattering angle must be assigned to USER_AP in USER_SCATANGLE. Several examples are provided in the next section.

\section{Appendix B.2 Example: Single Mechanism}

To ensure that the foregoing discussion is clear, we provide a simple example of a userdefined scattering mechanism. Let us assume that the user wishes to incorporate a scattering potential which is restricted to a spherical region of radius $R$ at location $\left(x_{0}, y_{0}, z_{0}\right)$ in the solid. Assume that the following can happen to any particle which enters this region: 1) it can be scattered by a mechanism with cross section $\sigma_{0} ; 2$ ) if scattered, it will suffer an energy loss of $\Delta E=\sqrt{E}$, where $E$ is the particle kinetic energy; 3) the particle will be then scattered into a random angle between 0 and $\pi$.

In this example, it is not necessary to make modifications to the subroutine USER_INITIALIZE, since no initialization prior to run-time is necessary. The subroutine USER_GETSIGS will test that the particle is in the scattering region and, if so, assign the cross section:

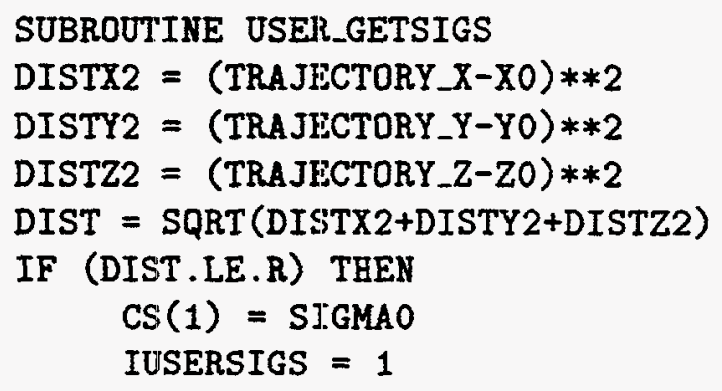


ELSE

IUSERSIGS $=0$
ENDIF
RETURA
END

The subroutines USER_ENERGYLOSS and USER_SCATANGLE are invoked if DISTANCE_TYPE decides that the user-defined scattering mechanism is found to occur. Thus, these userdefined routines do not need to perform a test that the particle is in the scattering region. Further, since there is only one user-defined scattering mechanism, information about which user-defined mechanism has occured (cf. Eq. (B.1.1)) is not needed. These routines might look as follows:

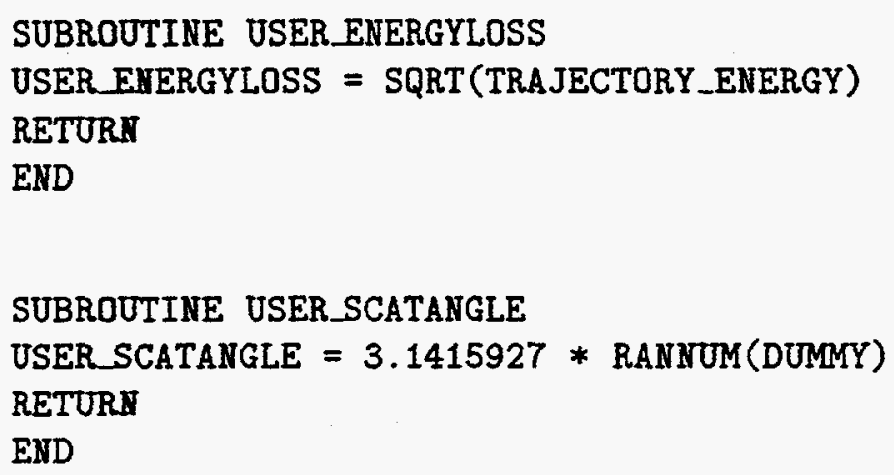

\section{Appendix B.2 Example: Multiple Mechanisms}

To complete our discussion of the addition of user-defined scattering mechanisms, we provide a more complicated example which involves multiple scattering mechanisms. Assume that the user has $N$ mechanisms which can occur, with cross sections given by

$$
\sigma_{i}=A_{i} r^{2}
$$

and energy loss per collision given by

$$
\Delta E=E_{i} r^{2}
$$

where $r$ is the distance from the particle to a point $\left(x_{0}, y_{0}, z_{0}\right)$ within the solid spherical region, and $N, A_{i}$ and $E_{i}$ are constants to be obtained via keyboard entry from the user. For simplicity we consider the scattering angle to be uniformly distributed on $[0, \pi]$. In this example there is need for pre-execution initialization, so USER_INITIALIZE must be modified. An example of such a modifcation is as follows:

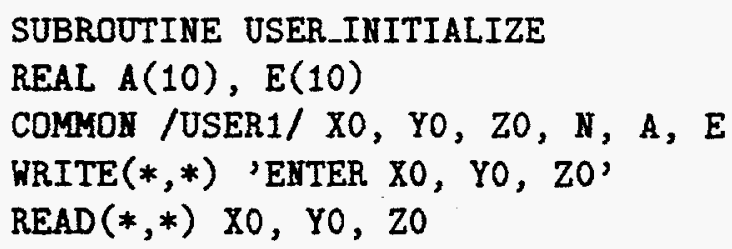




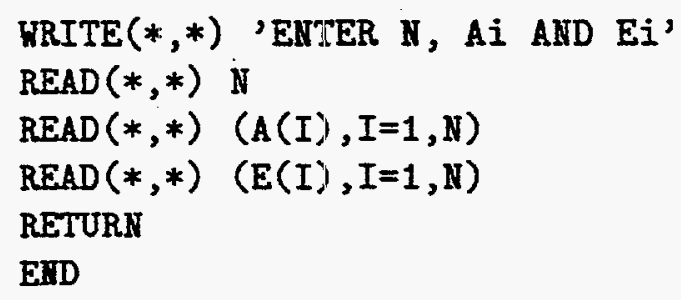

Here the common block USER1 is used to share information between the user-defined subroutines.

In this example, USER_GETSIGS has several cross sections to calculate,

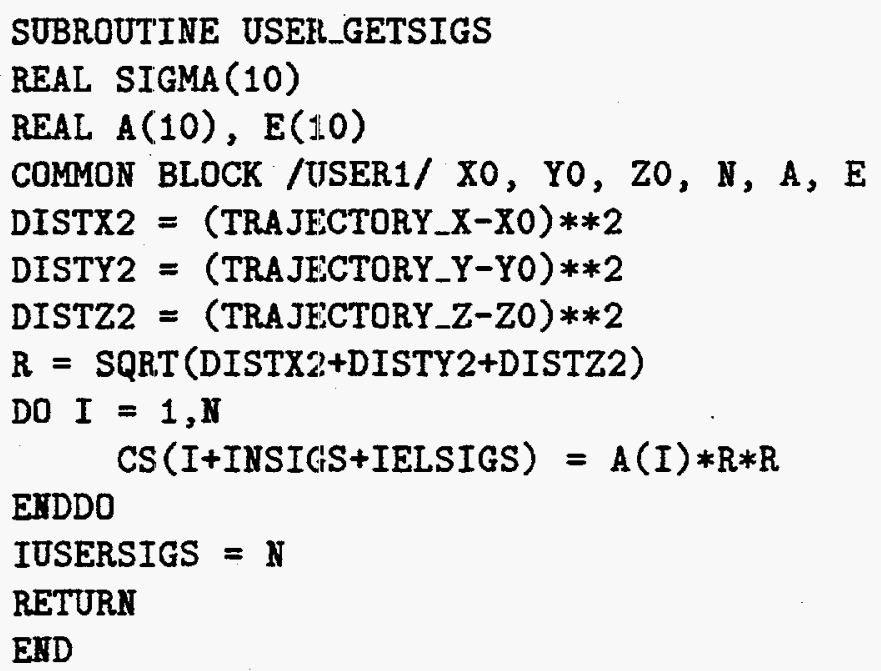

The key point of this subroutine is that the user-defined cross sections are stored after the external-datafile cross sections in array CS, via the array index I+INSIGS+IELSIGS.

In a similar manner, the type of user-defined scattering mechanism which has been determined to occur (in SPRITE subroutine DISTANCE_TYPE) is selected via

$$
\text { IUSERTYPE = ICURRENT_SCATTYPE - INSIGS - IELSIGS }
$$

as is shown in the following sample subroutine,

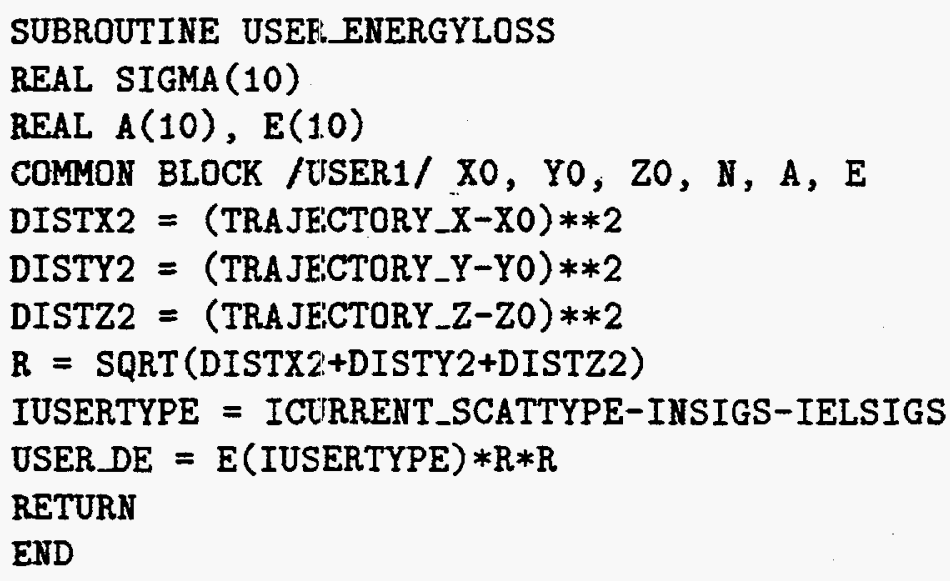


As specified above, the angular deflection per collision is to be randomly sampled on $[0, \pi]$,

SUBROUTIIE USER_SCATANGLE

USER_AP $=3.14159268 *$ RANNUM (DUMMY)

RETURN

END 


\section{Appendix C. User-Modifications to SPRITE}

It is possible for the user to make modifications to SPRITE, in addition to the inclusion. of a user-defined scattering mechanism (such as discussed in Appendix A). It is of course impossible to give other than general advice on how best to modify SPRITE to accomplish any given new task. It is possible, however, to provide several examples of ways in which SPRITE can be modified. It is hoped that these examples will illustrate the general process associated with making new modifications to SPRITE, as well as illuminate several important aspects of SPRITE which must be recognized and dealt with.

The following examples of modifications to SPRITE will be presented: 1) modifying the criteria for when a particle track is to be terminated (i.e., user-specification of when the simulation is to be stopped); 2) treatment of scattering between layers and other interfacial effects; and 3) addition of a spatially-localized scattering mechanism.

\section{Appendix C.1 New criteria for terminating the simulation}

The SPRITE program considers a scattering particle to be "stopped" when one of several conditions is met: the particle is transmitted through the last layer, the particle backscatters from the first layer, or particle energy falls below the stopping energy for the simulation. It is conceivable that the user may wish to incorporate additional criteria for terminating the simulation. Information about this type of modification is provided in this section.

The test that a particle has met "stopping" criteria is apparent as a decision-box in the program flow diagram in Figure 2.1. The subroutine which corresponds to this test is called PARTICLE_STOP, as can be seen in the main program code in Figure A.1. For convenience, this subroutine is presented in Figure C.1.

As can be seen in Figure C.1, the values of several of the global variables TRA JECTORY_D, TRA JECTORY ENERGY are checked. Depending on the outcome of the test, a flag STATUS is set so that the main calling program knows the results of the test (i.e., whether the particle is still "active" or has been stopped).

It is hoped that, at this point, the process required for modifying the stopping criteria is clear. Let us assume that the user wishes to add an additional criteria: stopping the simulation when the lifetime of the particle in the media exceeds a preset amount. This example might be relevant to the case of positrons in a solid, for example, which have a mean lifetime of approximately $100 \mathrm{ps}$ against annihilation with an electron in the host lattice. In this case, the user needs to add an additional ELSEIF clause to the stopping test in subroutine PARTICLE_STOP (Figure C.1); for example:

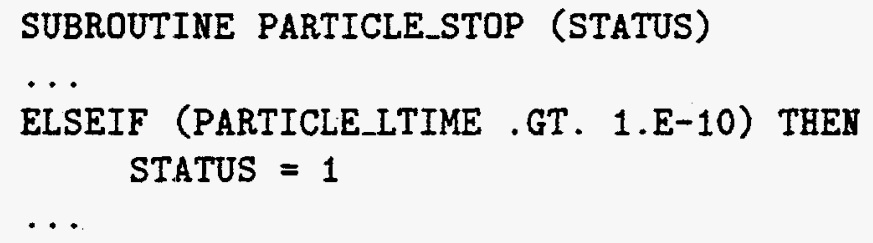




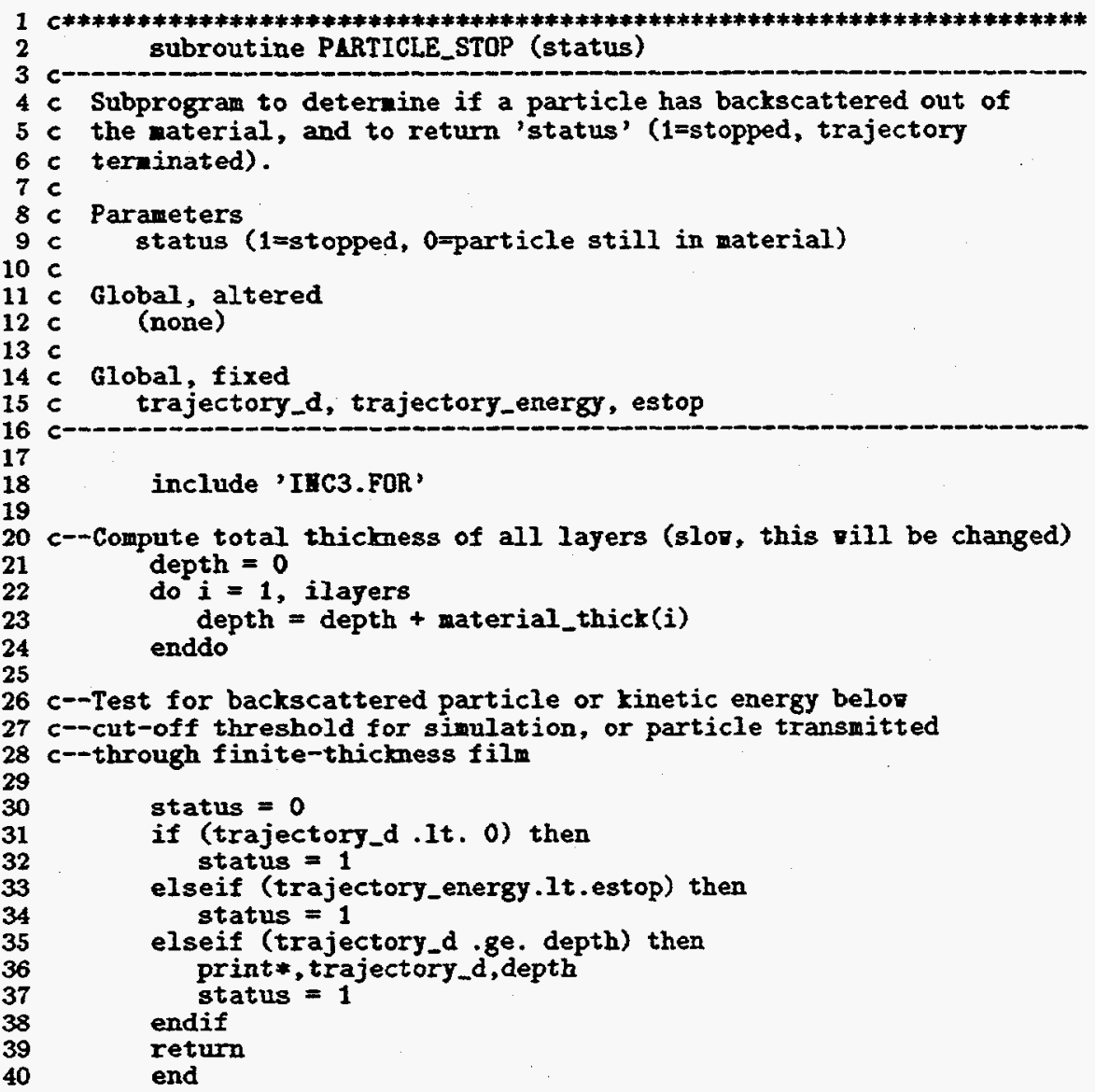

Figure C.1 The subroutine PARTICLE_STOP which determines when a scattering particle has met criteria for the termination of the scattering process.

Note that, due to the INCLUDE statement at the beginning of PARTICLE_STOP, all of the global variables are accessible in this subroutine, including the global variable PARTCLE_LTIME, which contains the current time spent by the current particle in the solid.

We give an additional example. Assume that a spherical void exists in the material (for example, in layer 2 at spatial coordinates $(110 \mathrm{~nm}, 120 \mathrm{~nm}, 130 \mathrm{~nm}$ ), with a radius of $10 \mathrm{~nm}$ ) and that a particle which randomly encounters this void will have some probability (say, $P$ ) of becoming permanently entrapped. In this case, the test for particle-track termination is slightly more complicated, but nevertheless follows the same scheme as above:

SUBROUTINE PARTICLE_STOP (STATUS) 


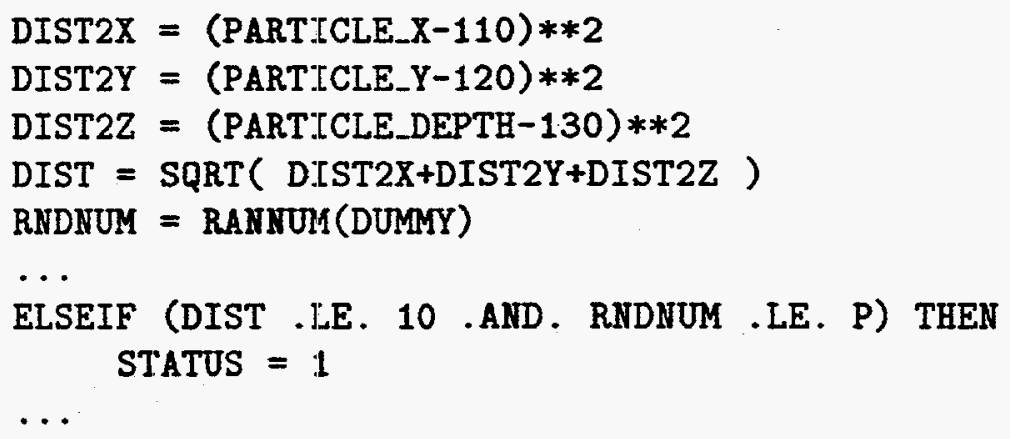

In this example, the distance from the particle to the void is calculated, and a stochastic test is included; thus, the simulation will be terminated only for a fraction $P$ of particles within $10 \mathrm{~nm}$ of the void. Note that the information that this void is located in layer 2 was not needed; particle coordinates are referenced with respect to the beginning of the first layer in a multilayer system, as shown in Figure 2.6; thus only the absolute coordinates of the void are necessary.

\section{Appendix C.2 Altering the Output Information}

To provide further insight into possible ways in which SPRITE can be modified, let us extend the foregoing example and assume that the user wishes to have a record of the number of annihilated particles displayed in the output file. Such a modification will involve three steps: 1) constructing; the stopping criteria; 2) establishing a new variable to store the number of annihilated particles; and 3) writing the value of this new variable to the output file. We have accomplished the first task in the example above. The introduction of a new global variable to the program can be accomplished in many ways; we recommend a procedure here: First, define a global variable in subroutine USER_INITIALIZE and assign this global variable to a common block (thus making it accessible to other subroutines in SPRITE). Recall that USER_INITIALIZE is executed prior to the start of the simulation, thus making this a logical point for the introduction of a new global variable. For example,

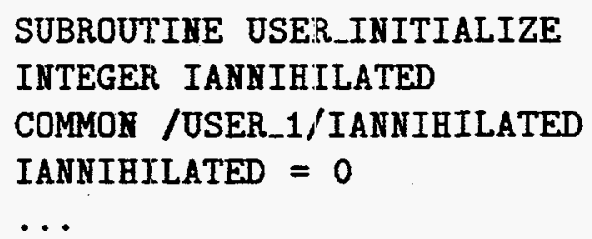

Next, secure access to this variable (IANYIHILATED) in subroutine PARTICLE_STOP, and increment the value of this variable when the annihilation criteria is met; for example:

SUBROUTINE PARTICLE_STOP

…

INTEGER IANHIHILATED

...

INCLUDE 'INC3.FOR'

-. 
COMMON /USER_1/IANHIHILATED

- .

ELSEIF (PARTICLELTIME .GT. 1.E-10) THEN

STATUS $=1$

IANNIHILATED = IANNIHILATED + 1

Finally, the subroutine which prepares the output file (GENERATE_REPORT) must be modified to write the value of variable IANNIHILATED to the output file.

\section{Appendix C.3 Interfacial Effects}

SPRITE performs the transport calculation for a collection of particles in (most generally) a lattice composed of several layers. In addition to scattering within a layer, due to the composition of the layer, it is natural to expect that there can be scattering between layers, due to the change in layer composition. Examples of such scattering include quantum mechanical barrier reflection, or particle trapping (localization) into a potential well at the interface.

The subroutine in SPRITE to handle the case when a particle has crossed an interface is called INTERFACE_ENCOUNTER, and the location of this subroutine can be found in the flow diagram in Figure D.2. Note that there is a possible source of confusion here in the relative positions of the two subroutines, UPDATE_TRAJECTORY and INTERFACE ENCOUNTER. The computation of the new particle position is sufficiently difficult that it is permitted the proceed first. Then, INTERFACE_ENCOUNTER can easily determine whether the particle has in fact crossed an interface, by comparing the new layer with the old. Then, if necessary, the particle position can again be updated, this time in subroutine INTERFACE_ENCDUNTER in response to physical interaction with the interface.

By default, SPRITE accepts one parameter which characterizes the interface (obtained from the simulation control data file by subroutine GETDATA). This parameter is then used by subroutine INTERFACE_ENCOUNTER. This value is assumed to be the magnitude of the work function at the interface. The probability $P$ of quantum mechanical barrier reflection from an energy step barrier is computed, and a fraction of incident particles $P$ are specularly reflected from the interface.

It is simple to modify this scheme. For example, assume that the user wishes to implement an interfacial trapping mechanism, whereby particles can be trapped into a potential well. For convenience, assume that the user has constructed an external function PROB_TRAP (ENERGY) which returns the probability that a particle of energy ENERGY will trap into this well. Then a possible modification to INTERFACE_ENCOUNTER might appear as in Figure C.2. Lines 6-7 are to determine that a particle has crossed a boundary. If a particle has crossed a boundary (line 8), and if is decided that the given particle will trap there (lines 9-11), then the depth coordinate of the interface is calculated (lines 13-15), and the global variable which stores the depth is update (line 16). Note that a logical flag is then set, presumably so that information that the particle is trapped can later be used to terminate the particle track in subroutine PARTICLE_STOP, as discussed in great deal above. 


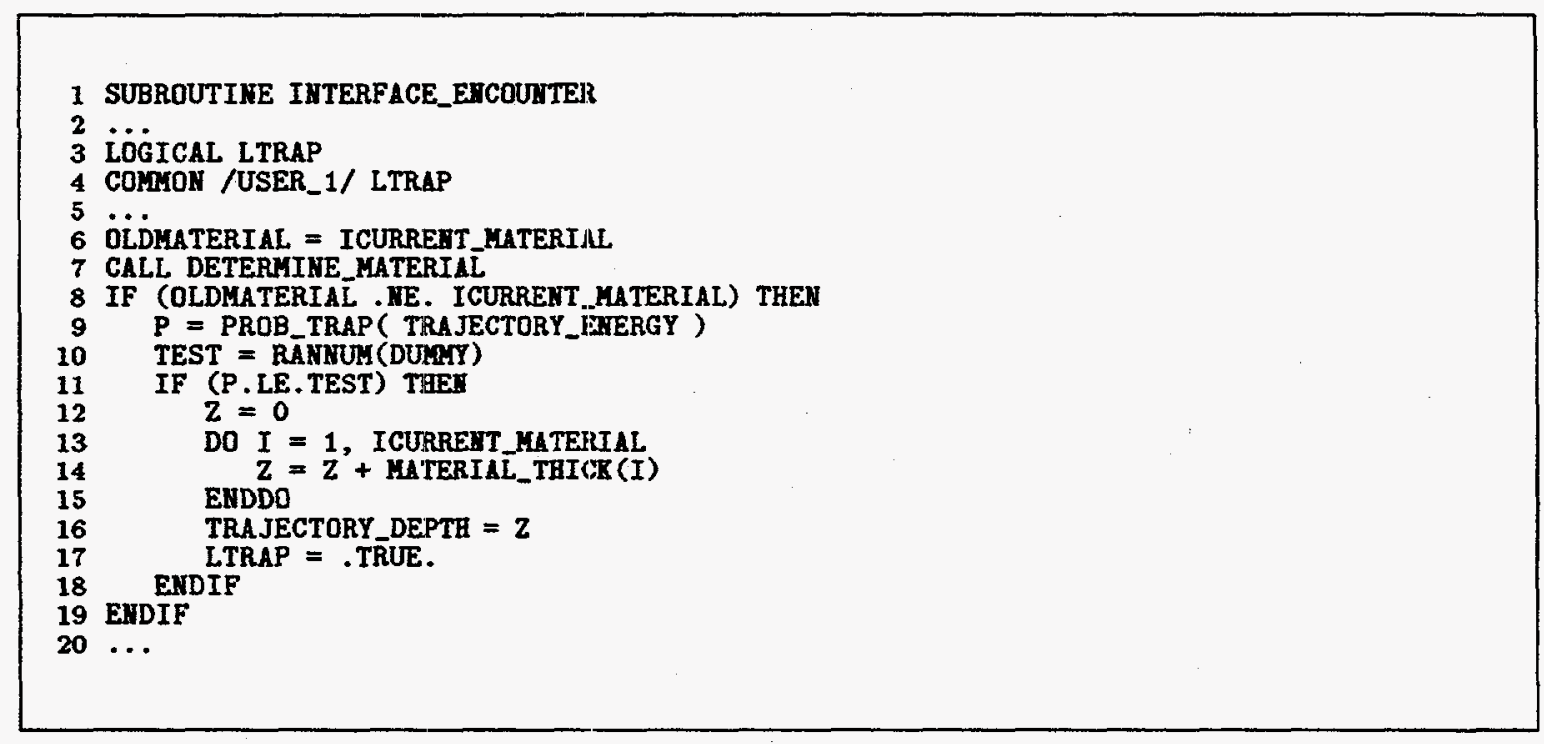

Figure C.2 A possible variation of IRTERFACE ENCOUNTER, modified by the user to confine a particle to an interface. Note that the global variable LTRAP and the function PROB_TRAP are user-defined and not part of SPRITE. 


\section{Appendix D. Useful Information}

This Appendix contains tables of information regarding the structure of SPRITE, expected to be of use to those intending to modify the program.

\begin{tabular}{|c|c|c|}
\hline $\begin{array}{ll}1 & c \\
2 & c \\
3 & c \\
4 & c \\
5 & c \\
6 & c \\
7 & c \\
8 & c \\
9 & c \\
10 & c \\
11 & c \\
12 & c \\
13 & c \\
14 & c \\
15 & c \\
16 & c \\
17 & c \\
18 & c \\
19 & c \\
20 & c \\
21 & c \\
22 & c \\
23 & c \\
24 & c \\
25 & c \\
26 & c \\
27 & c \\
28 & c \\
29 & c \\
30 & c \\
31 & c \\
32 & c \\
33 & c \\
34 & c \\
35 & c \\
36 & c \\
37 & c \\
38 & c \\
39 & c \\
40 & c\end{array}$ & 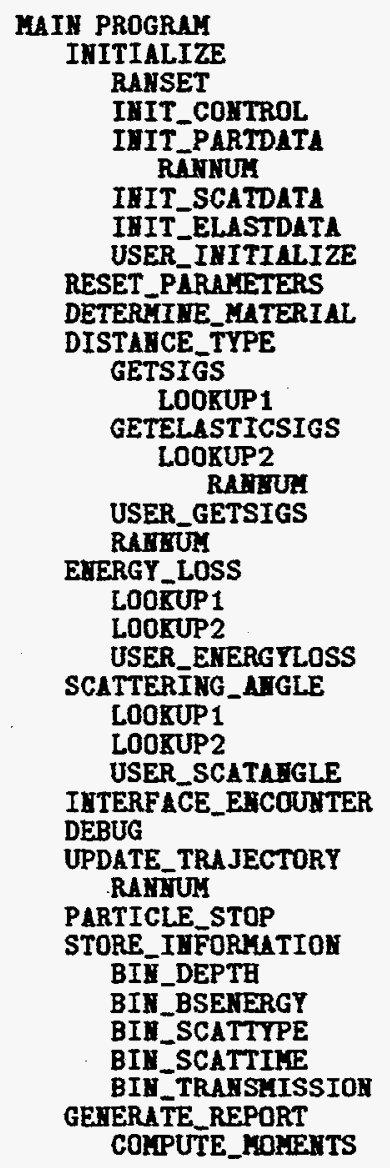 & 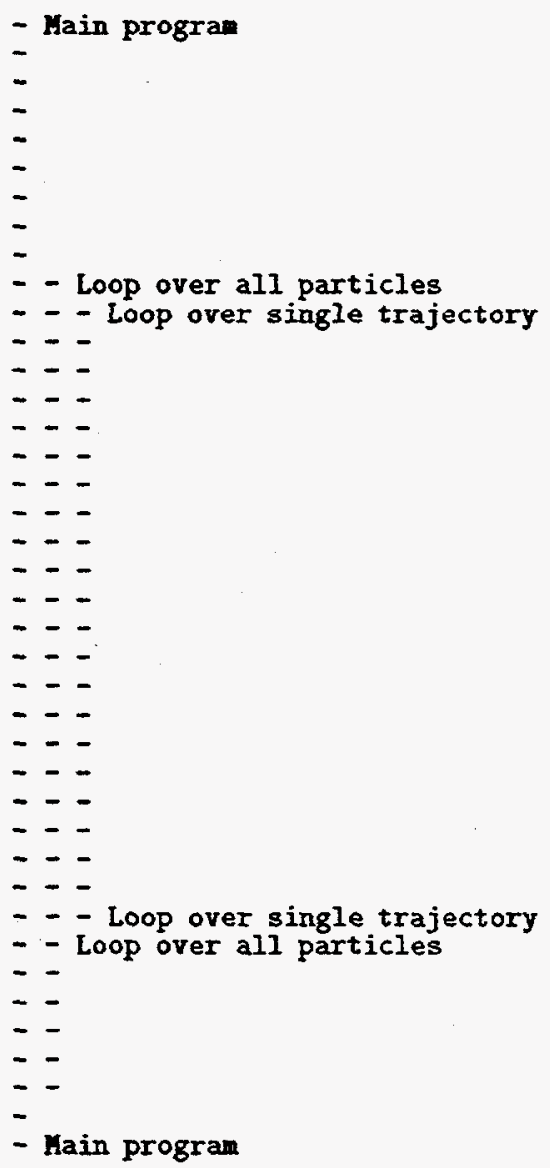 \\
\hline
\end{tabular}

Figure D.1 A complete hierarchical list of all routines in SPRITE. 
Main routines which control program flow

\section{DISTANCE_TYPE \\ ENERGY LOSS \\ SCATTERING_ANGLE UPDATE_LOCATION}

Select scattering type and mean free path Determine energy lost during a collision Determine scattering angle for a collision Update the scattering state $[\mathrm{r}(\mathrm{i}), \mathrm{E}(\mathrm{i}), \mathrm{D}(\mathrm{i})]$

Routines for program initialization
INITIALIZE
INIT_CONTROL
INIT_PARTDATA
INIT_TYPE1
INIT_TYPE2
USER_INITIALIZE

\begin{abstract}
Set parameters and global variables
Simulation control information

Initial particle distribution

Inelastic scattering information

Elastic-format scattering information

Obtain user-defined parameters
\end{abstract}

Routines for energy loss and angular deflection
ENERGYLOSS
Obtain energy loss information
USER_ENERGYLOSS
SCATTERING_ANGLE
User-defined energy loss information
USER_SCATANGLE
Obtain scattering angle information
User-defined scattering angle information

Routines for specific cross section calculations
GET_TYPE1
Inelastic cross sections
GET_TYPE2
Elastic-format cross sections
USER_GETSIGS
User-defined cross sections
LOOKUP1
Interpolate inelastic tables
LOOKUP2
Interpolate elastic (angular) tables

Routines to record simulation information

$\begin{array}{ll}\text { STORE_INFORMATION } & \text { Bin the stopping and energy distribution } \\ \text { BIN_DEPTH } & \text { Bin the stopping depth } \\ \text { BIN_BSENERGY } & \text { Bin the backscattered particle energy } \\ \text { BIN_SCATTYPE } & \text { Statistical record of scattering events } \\ \text { BIN_TRANSMISSION } & \text { Energy information upon transmission } \\ \text { GENERATE_REPORT } & \text { Prepare the output file }\end{array}$

Miscellaneous utility routines

RANNUM
RANSET
GGUBFS
COMPUTE_MOMENTS
PARTICLE_STOP

Return niformly distributed random number Set random number generation seed Linear congruential random number generator Statistical analysis of stopping profiles Checks conditions for ending the simulation

Table D.1 Grouping of subroutines in SPRITE by function. 


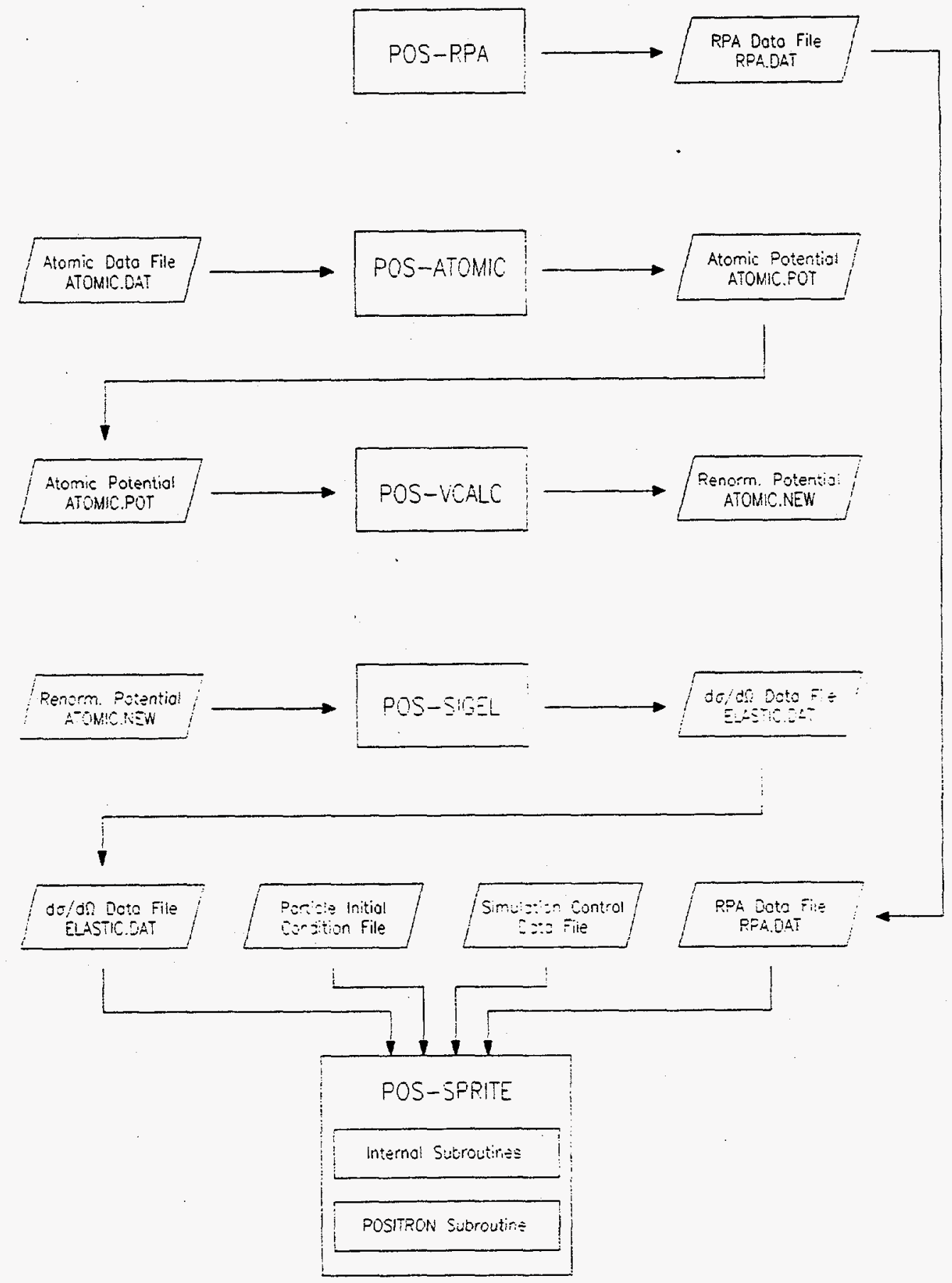

Figure D.2 Block diagram of the required files and ancilliary programs associated with the POS-SPRITE calculation. 
$\begin{array}{lll}\text { Variable } & \text { Description } & \text { Subroutine }\end{array}$

(Variables which describe the particle trajectory)

$\begin{array}{lll}\text { TRAJECTORY_ENERGY } & \text { particle energy } & \text { COMPUTE_ENERGY } \\ \text { TRAJECTORY_X } & \text { x-coordinate } & \text { UPDATE_LOCATION } \\ \text { TRAJECTORY_Y } & \text {-coordinate } & \text { UPDATE_LOCATION } \\ \text { TRAJECTORY_Z } & \text { z-coordinate } & \text { UPDATE_LOCATION } \\ \text { TRAJECTORY_LTOT } & \text { mean free path } & \text { DISTANCE_TYPE } \\ \text { TRAJECTORY_ELOSS } & \text { energy loss } & \text { COMPUTE_ENERGY }\end{array}$

(Description of the materials and layers)

\author{
ILAYERS \\ IMECH \\ IELMECH \\ MAT_TITLE \\ MAT_PARTICLEMASS \\ MAT_ATMASS \\ MAT_DENSITY \\ MAT_THICK \\ MAT_WORKFUN \\ MAT_MFP2SIG
}

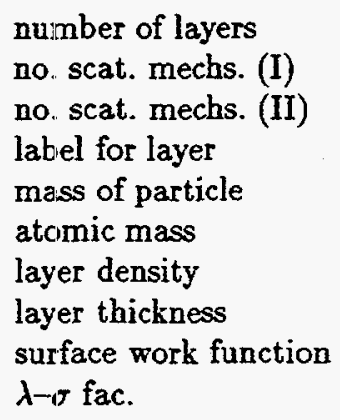

(The variables which store interaction information)

$\begin{array}{lll}\text { TYPE1_E } & \text { energy grid } & \text { INITIALIZE } \\ \text { TYPE1_MFP } & \text { mean free path } & \text { INITIALIZE } \\ \text { TYPE1_DE } & \text { energy loss } & \text { INITIALIZE } \\ \text { TYPE1_DT } & \text { scattering angle } & \text { INITIALIZE } \\ \text { TYPE2_EV } & \text { energy grid } & \text { INITIALIZE } \\ \text { TYPE2_DSIG } & \text { diff. cross section } & \text { INITIALIZE } \\ \text { TYPE2_DE } & \text { energy loss } & \text { INITIALIZE } \\ \text { TYPE2_DT } & \text { scattering angle } & \text { INITIALIZE }\end{array}$

Table D.2 The important global variables contained in SPRITE, their function, and the subroutine in which their value is first established. 
$\begin{array}{lll}\text { Variable Description } & \text { Subroutine }\end{array}$

(The histograms into which endpoint coordinates are binned)

XBIN
YBIN
ZBIN
EBIN
XBINSTART
XBINSTOP
XBINNUM

XBIN

YBIN

ZBIN

EBIN

XBINSTOP

XBINNUM

\author{
$\mathrm{x}$-position histogram \\ $y$-position histogram \\ z-position histogram \\ backscattered histogram \\ hist. starting coord. \\ hist. ending coord. \\ no. histogram bins
}

\author{
STORE_INFORMATION \\ STORE INFORMATION \\ STORE_INFORMATION \\ STORE_INFORMATION \\ INITIALIZE \\ INITIALIZE \\ INITIALIZE
}

(Variables which store statistical information about the particle trajectories)

HIST_SCATTIME
HIST_TOTALTIME
HIST_DEPTHCOUNTER
HIST_SCATBIN
HIST_SCATCOUNTER
HIST_BACKCOUNTER

HIST SCATTIME

HIST_TOTALTIME HIST_SCATBIN HIST BACKCOLNTER time since last event total lifetime during scattering no. particles which stop record of no. scattering types no. scattering events no. particles which backscatter
STORE_INFORMATION STORE.INFORMATION STORE_INFORMATION STORE_INFORMATION STORE_INFORMATION STORE_INFORMATION

Table D.3 The important statistical variables contained in SPRITE, their function, and the subroutine in which their value is first established. 


\section{Appendix E. Sample Output Information}

This Appendix contains sample SPRITE input files and the corresponding output file.

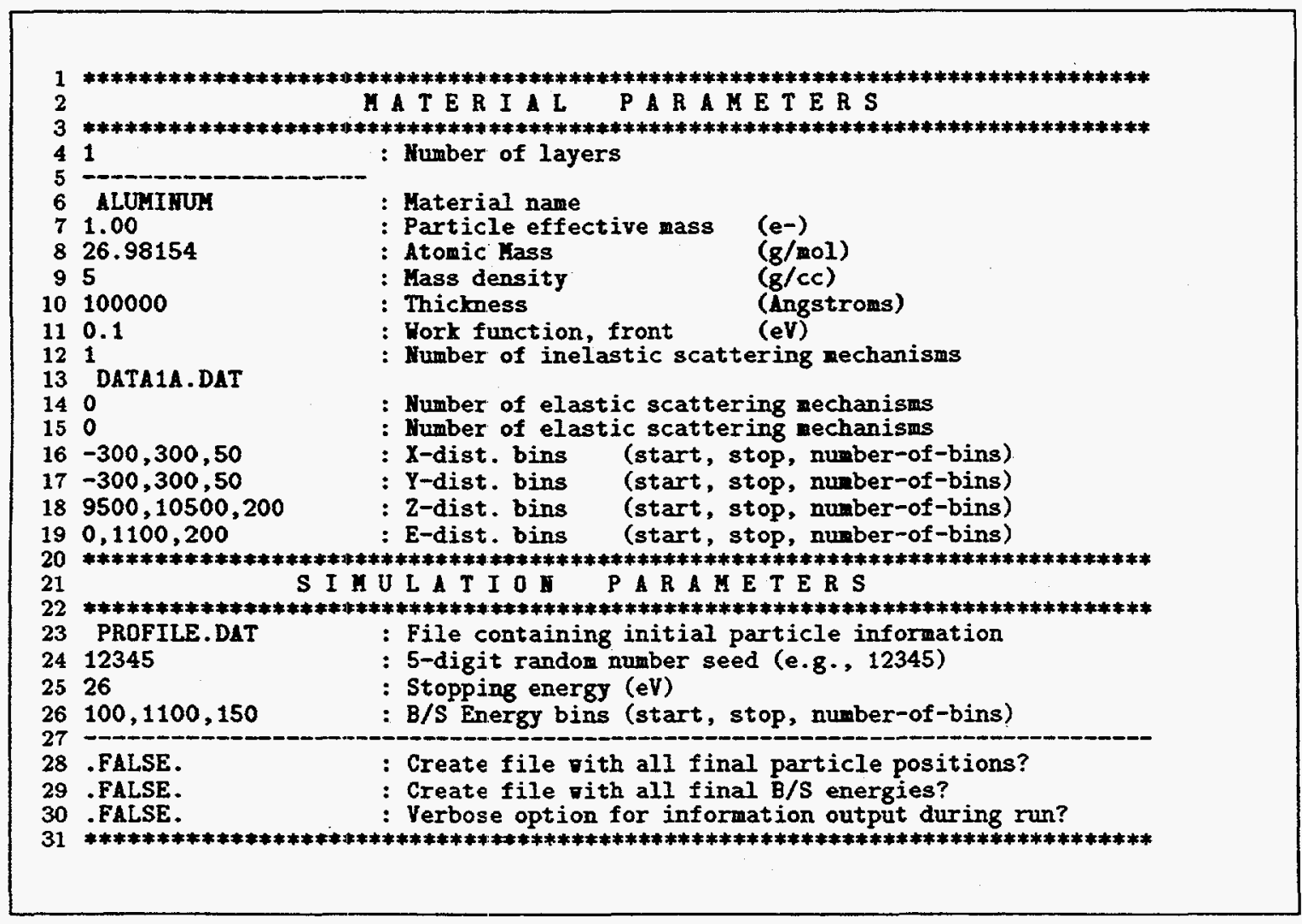

Figure E.1 A sample simulation control data file, which specifies that the particles will scatter within a single layer of $\mathrm{Al}$. 


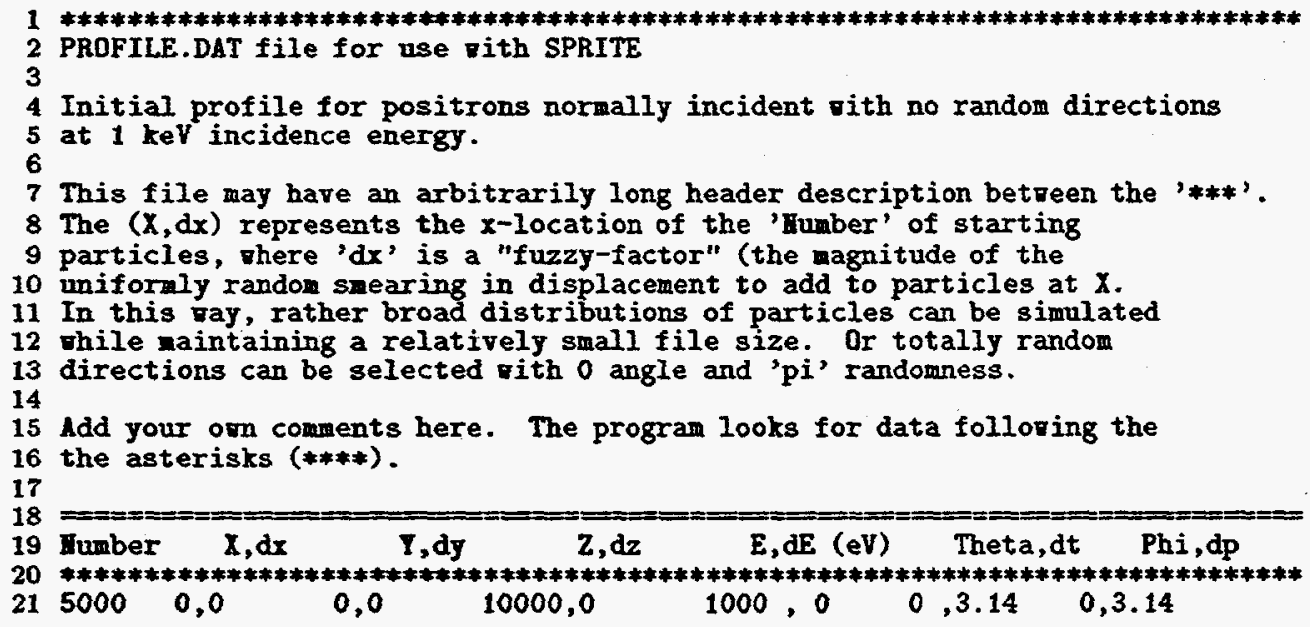

Figure E.2 A sample particle initial condition data file, which specifies that the 5000 particles, beginning deep within the solid and with random initial directions. 


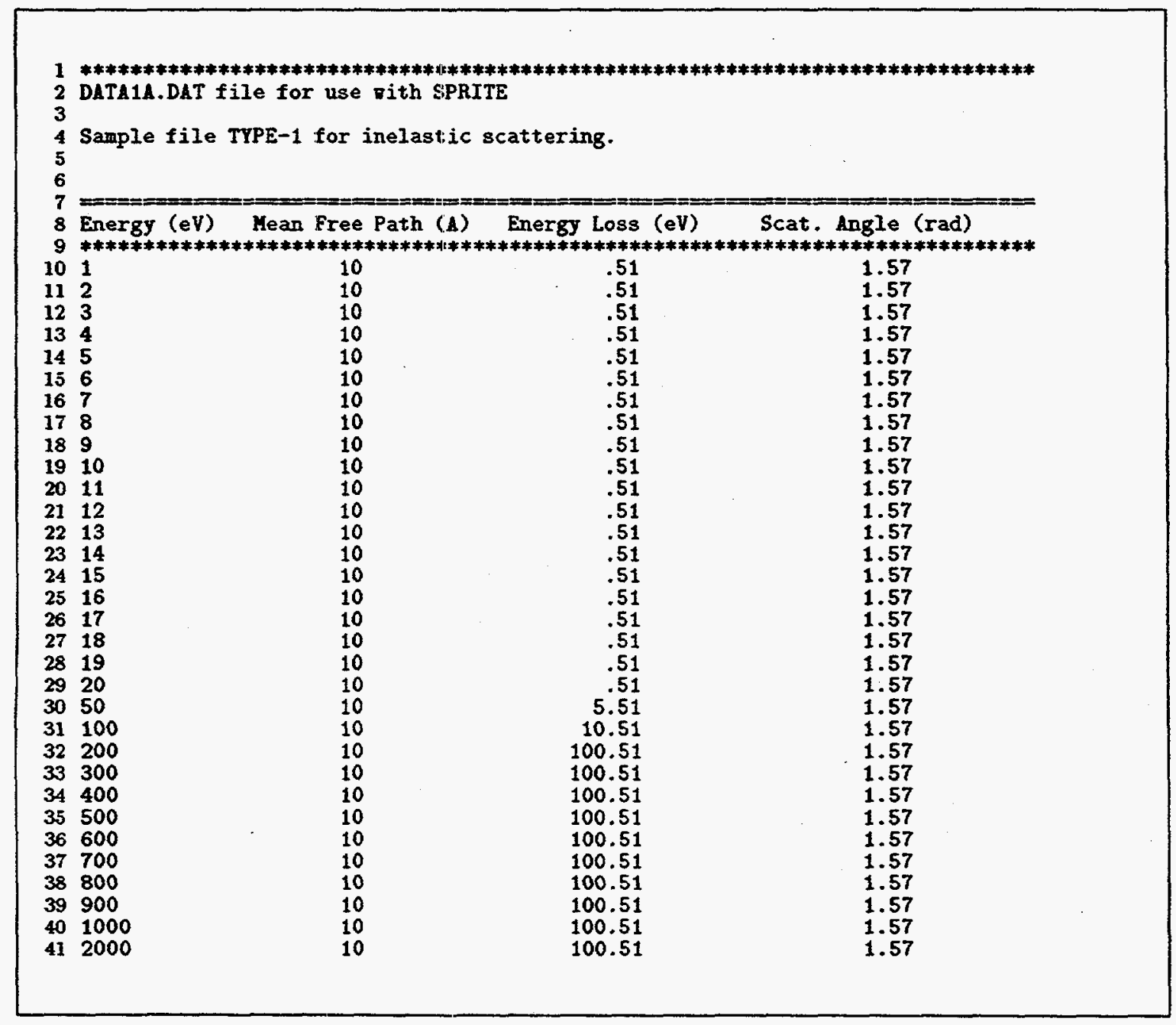

Figure E.3 A sample interaction file, which specifies the energy-dependent mean free path, mean energy loss per interaction, and mean scattering angle per interaction for the particles. 


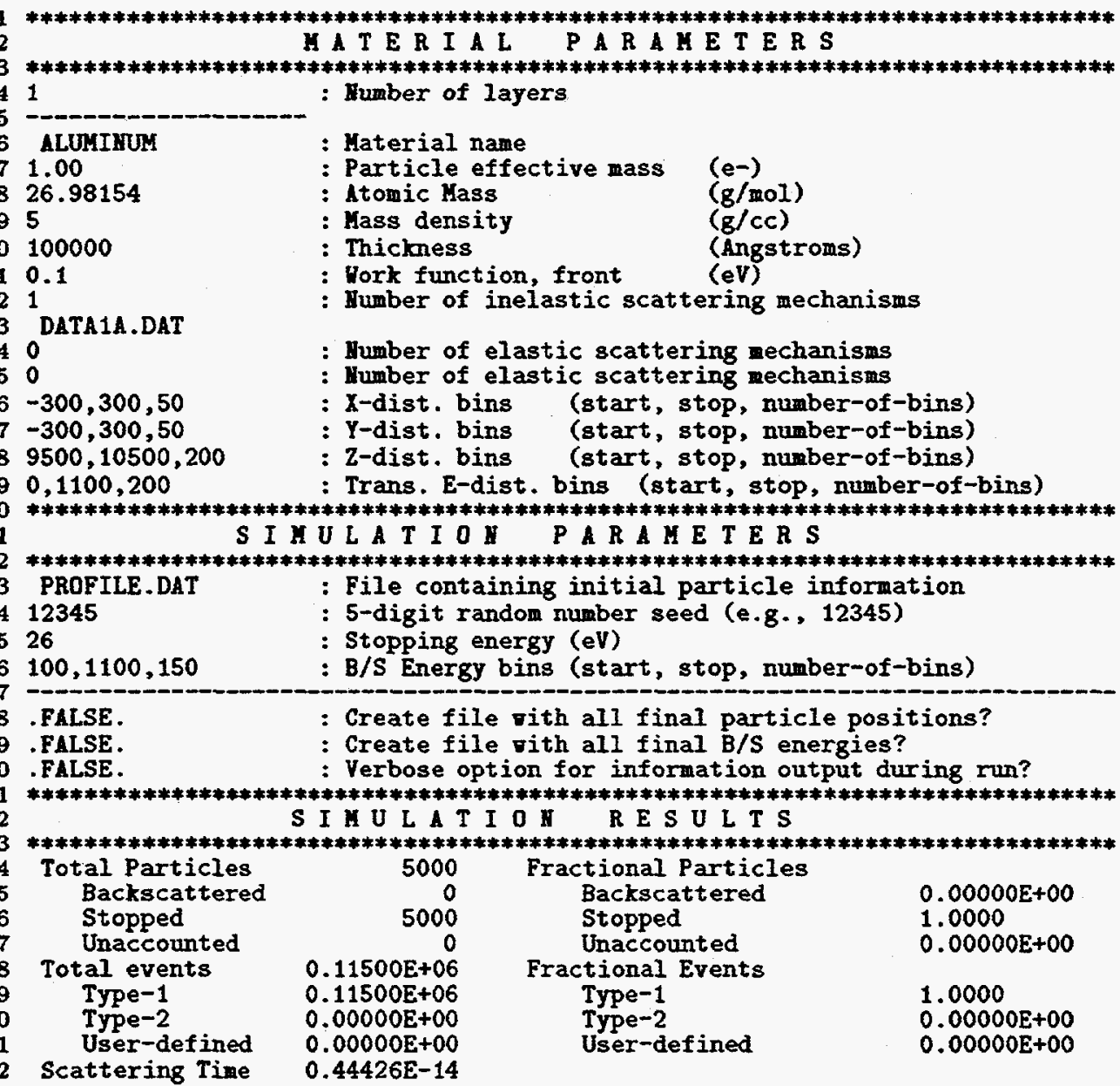

Figure E.4 A sample output data file, which contains physical information about the endpoint distributions of the scattering process, as well as statistical information accumulated during the interaction history. 


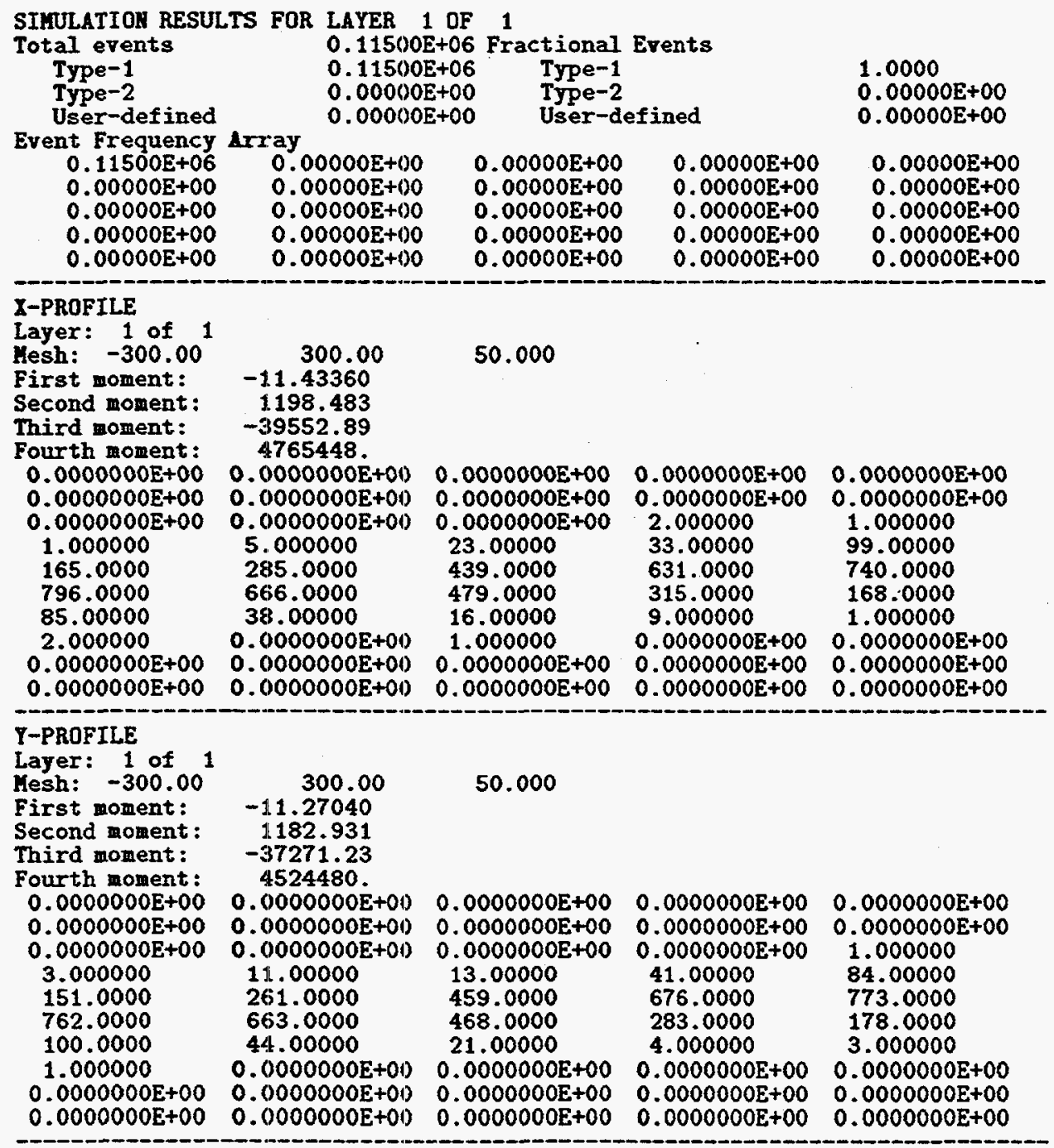

Figure E.5 A sample output file, cont'd. Here is shown the statistical information about the scattering process. 


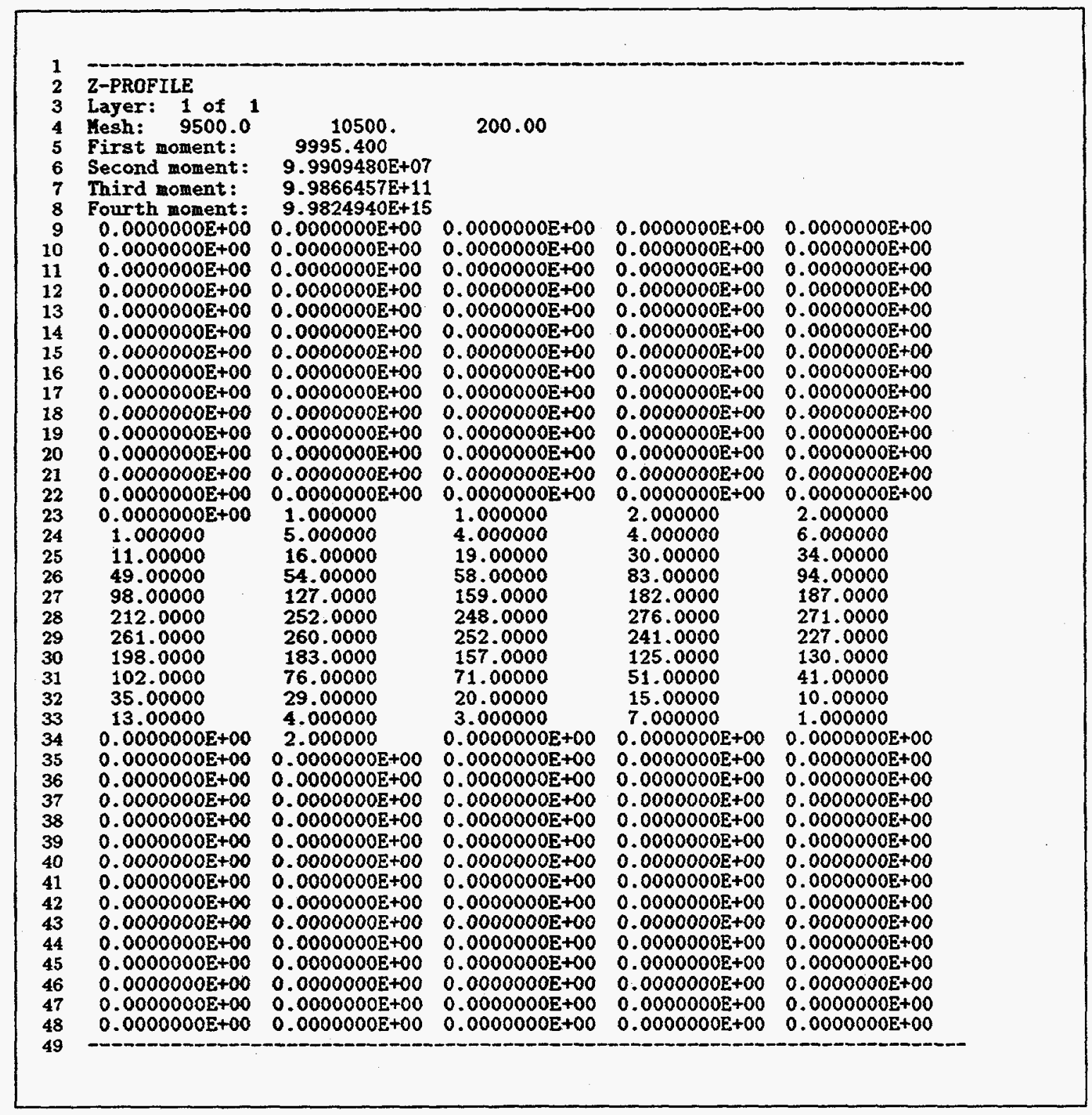

Figure E.6 A sample output file, cont'd. This is the depth distribution of the particles, approximately Gaussian because the particles were incident from a point source and scattered at random directions. 


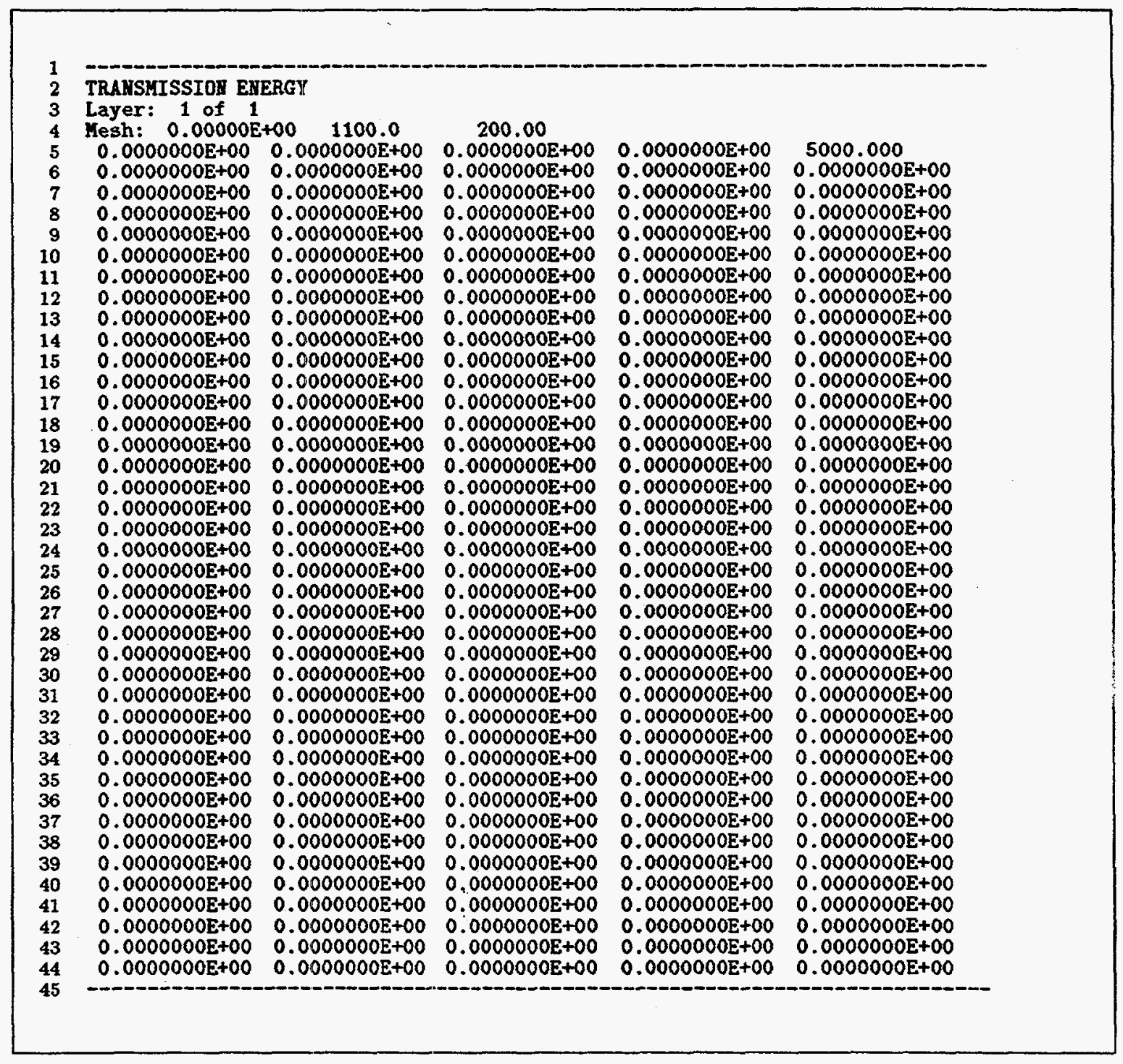

Figure E.7 A sample output file, cont'd. This is the energy distribution of the transmitted particles. In this case, the particles began from a point source deep within the material, and were stopped by insufficient energy before reaching the outer surface, thus no particles were transmitted. 


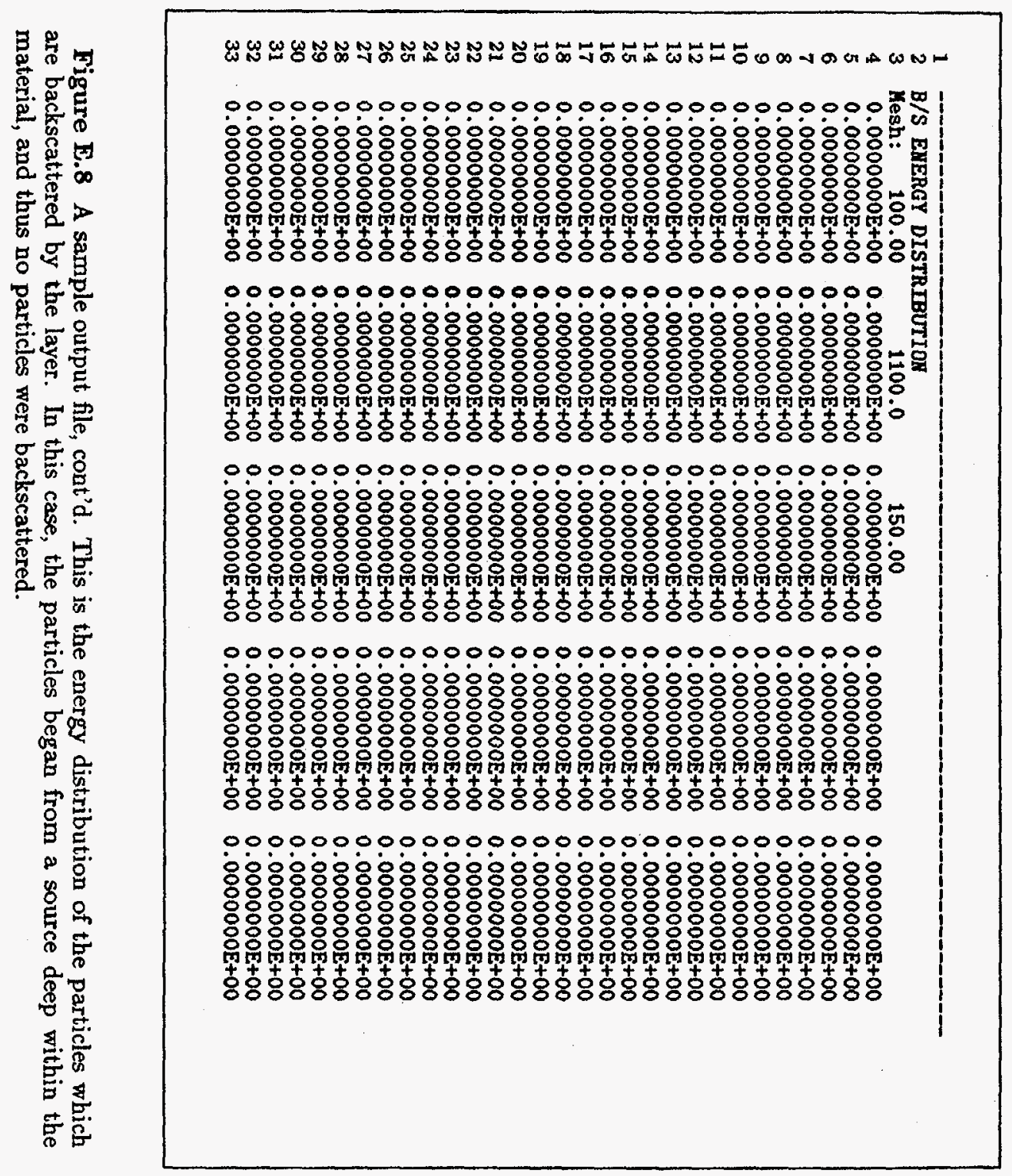




\section{References}

[1] V. J. Ghosh and G. C. Aers, Phys. Rev. B 51, 45 (1995).

[2] V. J. Ghosh, M. McKeown, K. G. Lynn, and D. O. Welch, Nuc. Instr. Meth. Phys. Res. B 90, 442 (1994).

[3] V. J. Ghosh, K. G. Lynn, and. D. O. Welch, Proc. Int'l. School Physics: Enrico Fermi CXXV, 683 (1995).

[4] G. J. van der Kolk, B. Nielsen, V. J. Ghosh, and K. G. Lynn, preprint.

[5] V. J. Ghosh, M. McKeown, D. O. Welch, and K. G. Lynn, preprint.

[6] M. McKeown, K. G. Lynn, and D. O. Welch, Technical report, Brookhaven National Laboratory (unpublished).

[7] P. Asoka-Kumar and K. G. Lynn, App. Phys. Lett. 57, 1634 (1990).

[8] A. Weiss et al., Rad. Phys. Chem. 44, 665 (1994).

[9] K. A. Ritley, K. G. Lynn, V. G. Ghosh, and D. O. Welch, in Positron Beams for Solids and Surfaces, edited by E. Ottewitte and A. H. Weiss (American Institute of Physics, New York, 1992), pp. 64-72.

[10] V. G. Ghosh, D. O. Welch, and K. G. Lynn, in Positron Beams for Solids and Surfaces, edited by E. Ottewitte and A. H. Weiss (American Institute of Physics, New York, 1992), pp. 37-47.

[11] K. A. Ritley, M. McKeown, and K. G. Lynn, in Positron Beams for Solids and Surfaces, edited by P. Schultz, G. R. Massoumi, and P. J. Simpson (American Institute of Physics, New York, 1990), pp. 3-18.

[12] K. A. Ritley, V. J. Ghosh, K. G. Lynn, M. McKeown and D. O. Welch, J. App. Phys. 74, 3479 (1993).

[13] K. F. Canter, in Positron Beams for Solids and Surfaces, edited by P. Schultz, G. R. Massoumi, and P. J. Simpson (American Institute of Physics, New York, 1990), p. 53.

[14] A. Weiss, in Positron Beams for Solids and Surfaces, edited by P. Schultz, G. R. Massoumi, and P. J. Simpson (American Institute of Physics, New York, 1990), p. 61.

[15] P. J. Schultz and K. G. Lynn, Rev. Mod. Phys. 60, 701 (1988).

[16] R. M. Nieminen and J. Oliva, Phys. Rev. B 22, 2226 (1980).

[17] B. Bergersen and E. Pajanne, App. Phys. 4, 25 (1974).

[18] B. Bergersen et al., Solid State Comm. 15, 1377 (1974). 
[19] W. Brandt and N. R. Arista, Phys. Rev. A 19, 2317 (1979).

[20] W. Brandt, App. Phys. 5, 1 (1974).

[21] K. G. Lynn, D. O. Welch, J. Throwe, and B. Nielsen, Intr. Mat. Rev. 36, 1 (1991).

[22] A. P. Mills and R. J. Wilson, Phys. Rev. A 26, 490 (1982).

[23] S. Valkealahti and R. M. Nieminen, App. Phys. A 32, 95 (1983).

[24] S. Valkealahti and R. M. Nieminen, App. Phys. A 35, 51 (1984).

[25] H. A. Bethe, Handb. Phys. 24, 273 (24).

[26] The EGS4 Code System, Stanford University, 1985.

[27] The MORSE Monte Carlo radiation transport code system, Oak Ridge National Laboratory, 1975.

[28] J. Ziegler, The stopping power and range of ions in solids (Pergamon Press, New York, 1985), Vol. 4.

[29] J. Biersack and L. Haggmark, Nuc. Instr. Meth. Phys. Res. 174, 257 (1980).

[30] W. R. Nelson and T. M. Jenkins, Computer Techniques in Radiation Transport and Dosimetry (Plenum Press, New York, 1980).

[31] J. D. Martinez, R. Mayol, and F. Salvat, J. App. Phys. 67, 2955 (1990).

[32] I. Adesida, R. Shimizu, and T. E. Everhart, J. App. Phys. 51, 5962 (1980).

[33] A. Desalvo, A. Parisini, and R. Rosa, J. Phys. D 17, 2455 (1984).

[34] F. Salvat, R. Mayol, and J. D. Martinez, J. Phys.B 20, 6597 (1987).

[35] D. Liljequist, F. Salvat, R. Mayol, and J. D. Martinez, J. App. Phys. 65, 2431 (1989).

[36] A. J. Green and R. C. G. Leckey, J. Phys. D 9, 2123 (1976).

[37] F. Salvat and J. Parellada, J. Phys. D 17, 1545 (1984).

[38] R. Shimuzu, Y. Katoaka, and T. Matsukawa, J. Phys. D 8, 820 (1975).

[39] R. Shimuzu, Y. Katoaka, T. Koshikawa, and H. Hashimoto, J. Phys. D 9, 101 (1976).

[40] M. Kotera, K. Murata, and K. Nagami, J. App. Phys. 52, 997 (1981).

[41] P. Miller, J. M. Gibson, A. Bleeker, and J. Liddie, J. Vac. Sci. Tech. B11, 2352 (1993).

[42] D. F. Kyser, in Introduction to analytical electron microscopy, edited by J. Hren, J. Goldstein, and D. Joy (Plenum, New York, 1979), pp. 199-221.

[43] F. W. Reif, Introduction to statistical and thermal physics (McGraw-Hill, New York, 1965), pp. 565-567.

[44] J. W. Williamson and G. C. Duncan, Am. J. Phys. 54, 262 (1986).

[45] S. E. Koonin, Computational physics (Benjamin, New York, 1986). 
[46] M. Abramowitz and I. A. Stegun, Handbook of mathematical functions (National Bureau of Standards, Washington, 1970).

[47] R. Shankar, Principles of quantum mechanics (Plenum, New York, 1980), p. 183.

[48] N. Ozturk, W. Williamson, and A. Antolak, J. App. Phys. 71, 11 (1992).

[49] N. Ozturk, W. Williamson, and A. Antolak, J. App. Phys. 70, 537 (1992).

[50] D. McGarrah, A. Antolak, and W. Williamson, J. App. Phys. 69, 6812 (1991).

[51] R. Meredith et al., J. App. Fhys. 68, 4937 (1990).

[52] A. Antolak and W. Williamson, J. App. Phys. 69, 3760 (1991).

[53] D. A. Liberman, D. T. Cromer, and J. T. Waber, Comp. Phys. Comm. 2, 107 (1971).

[54] D. A. Liberman, J. T. Waber, and D. T. Cromer, Phys. Rev. 137, A27 (1965).

[55] H. Kolbenstvedt, J. App. Phys. 38, 4785 (1967).

[56] J. M. Jauch and F. Rohrlich, The theory of photons and electrons (Addison-Wesley, Cambridge, 1954).

[57] C. Moller, Ann. Physik 14, 531 (1932).

[58] J. Bhabha, Proc. Roy. Soc. (London) A154, 195 (1936).

[59] F. Rohrlich and B. C. Carlson, Phys. Rev. 93, 38 (1954).

[60] G. P. Williams, Electron binding energies for the elements, Brookhaven National Laboratory (1983).

[61] M. Gryzinski, Phys. Rev. 138, 305 (1965).

[62] M. Gryzinski, Phys. Rev. A 138, 322 (1965).

[63] M. Gryzinski, Phys. Rev. A 138, 336 (1965).

[64] J. J. Quinn, Phys. Rev. 126, 1453 (1962).

[65] D. R. Penn, Phys. Rev. B 13, 5248 (1976).

[66] C. J. Powell, Surf. Sci. 44, 29 (1974).

[67] J. C. Ashley, J. Electron Sper. and Related Phenom. 50, 323 (1990).

[68] J. C. Ashley, J. App. Phys. 69, 674 (1991).

[69] J. C. Ashley, C. J. Tung, and R. H. Ritchie, Surf. Science 81, 409 (1979).

[70] D. R. Penn, Phys. Rev. 35, 482 (1987).

[71] C. L. Foiles, in Landolt-Bornstein (Springer-Verlag, Berlin, 1985), Chap. 4.

[72] J. Oliva, Phys. Rev. B 21, 4925 (1980).

[73] J. Oliva, Phys. Rev. B 21, 4909 (1980). 
[74] J. Oliva, Phys. Rev. B 21, 4918 (1980).

[75] D. Isaacsen, Technical report, New York University (unpublished).

[76] D. Pines and P. Nozieres, Quantum Theory of Liquids (Plenum, New York, 1969), Vol. 1.

[77] G. D. Mahan, Many-particle physics (Plenum, New York, 1990).

[78] N. W. Ashkroft and N. D. Mermin, Inroduction to solid state physics (Saunders, Philadelphia, 1976).

[79] D. Pines, Rev. Mod. Phys. 28, 184 (1956).

[80] D. Pines and D. Bohm, Phys. Rev. 85, 338 (1952). 